\title{
Spectroscopic and Computational Investigations of the Thermodynamics of Boronate Ester and Diazaborole Self-Assembly.
}

\author{
Alexander R. Goldberg and Brian H. Northrop* \\ Wesleyan University, Department of Chemistry \\ Hall-Atwater Laboratories, 52 Lawn Ave., Middletown, CT 06459, USA \\ Email: bnorthrop@wesleyan.edu, phone: 860-685-3987
}

\section{SUPPORTING INFORMATION}

(47 pages)

I. ${ }^{1} \mathrm{H}$ and ${ }^{13} \mathrm{C}$ NMR Spectra of 11a-f and 12a-f

S2-S13

II. Analytical ${ }^{1} \mathrm{H}$ Spectra of Boroxine Anhydrides

S14-S19

III. ${ }^{1}$ H NMR Spectra of Equilibrated Boronate Esters

S20-S22

IV. ${ }^{1}$ H NMR Spectra of Equilibrated Diazaboroles

S23-S25

V. Concentration and $K_{\mathrm{eq}}$

S26-S28

VI. Boronic Acid Conformations \& Relative Energetics

S29-S30

VII. Bond Lengths of 12a-f, 13a-f, and 14a-f

VIII. Calculated Electron Density Maps

IX. Stationary Point Coordinates \& Energies

X. Supporting References 


\section{I. ${ }^{1} \mathrm{H}$ and ${ }^{13} \mathrm{C}$ NMR Spectra of 11a-f and 12a-f}

11a<smiles>COc1ccc(B2Oc3ccc(C(C)(C)C)cc3O2)cc1</smiles>
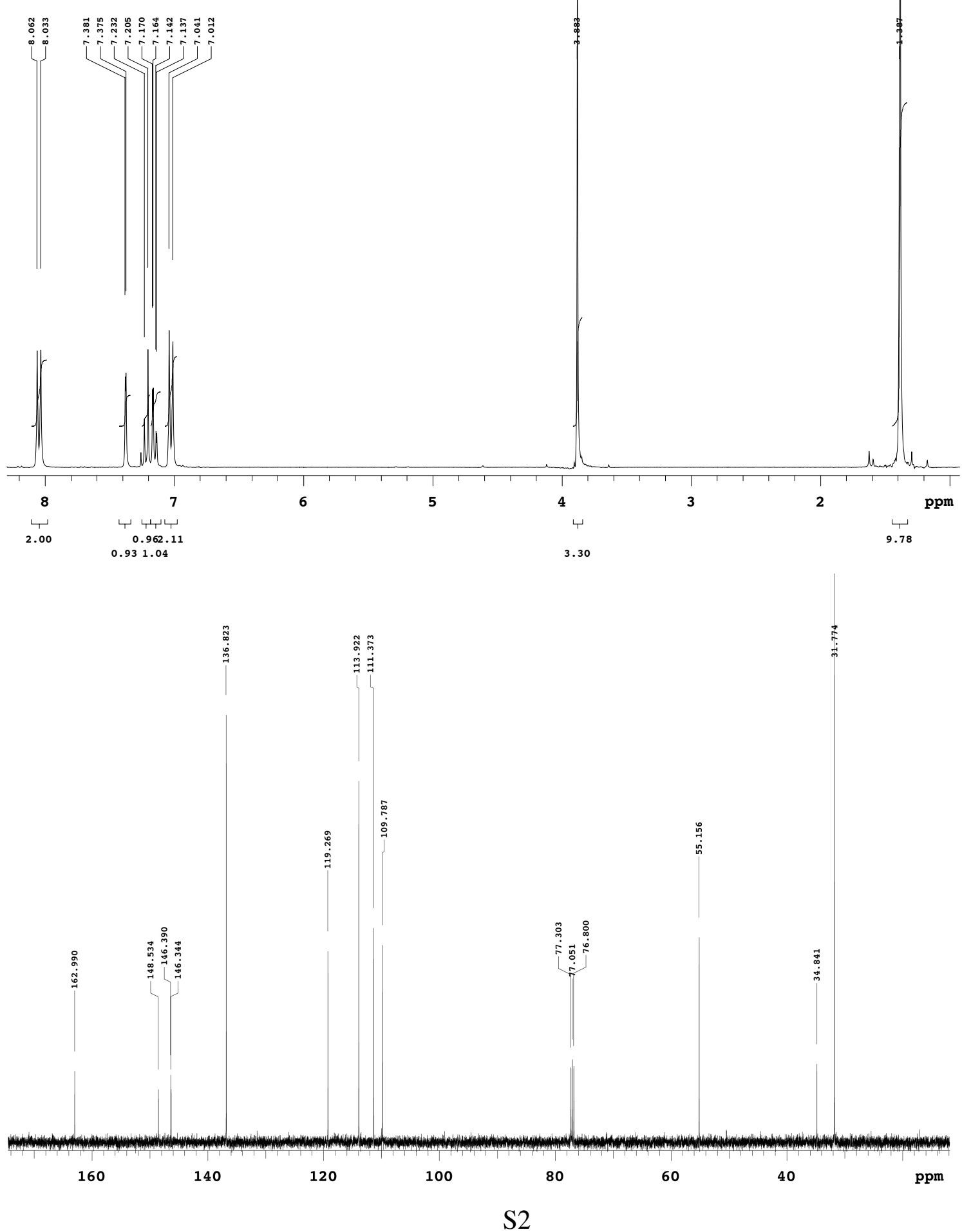
11b<smiles>CC(C)(C)c1ccc(B2Oc3ccc(C(C)(C)C)cc3O2)cc1</smiles>
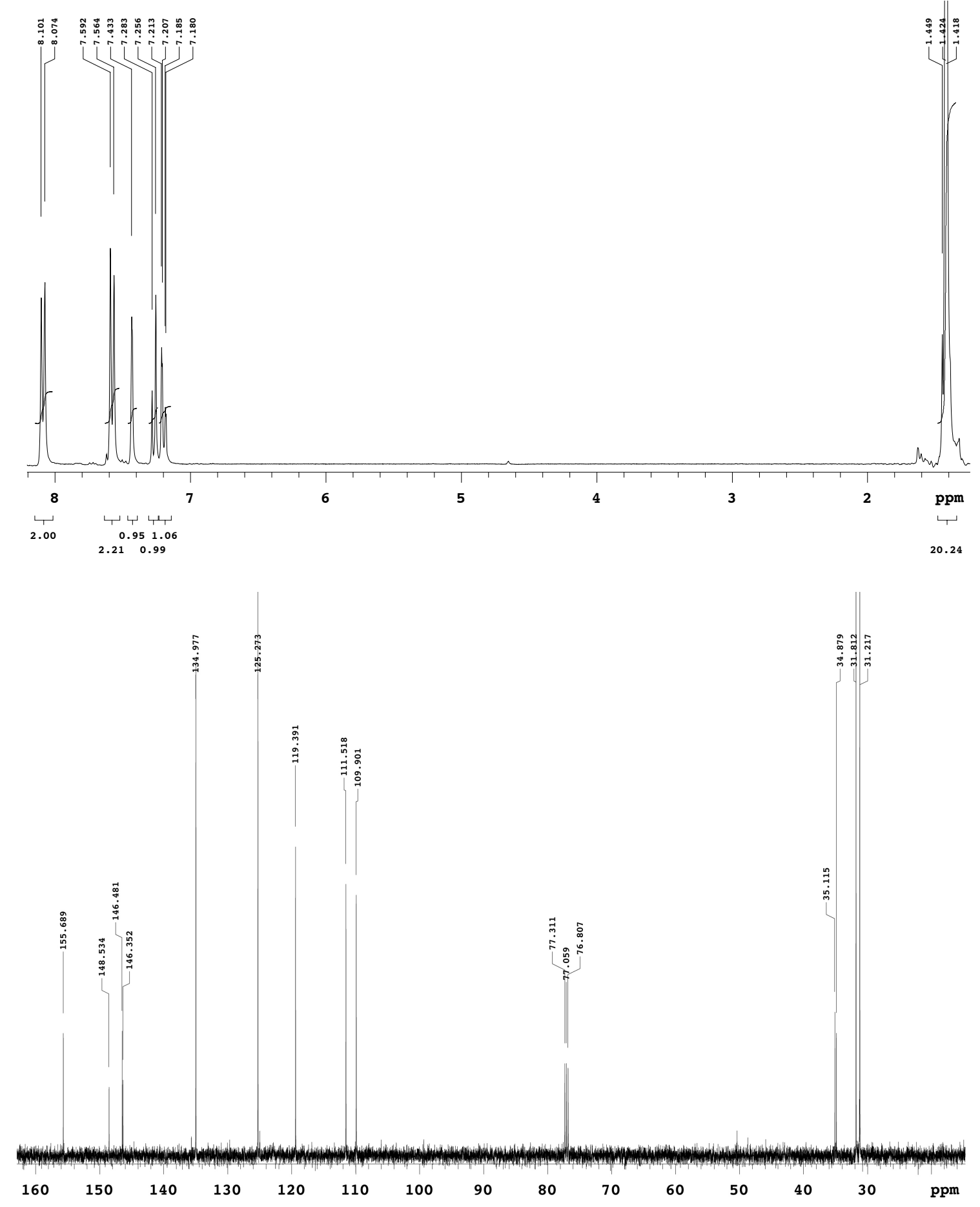
$11 c$<smiles>CC(C)(C)c1ccc2c(c1)OB(c1ccccc1)O2</smiles>
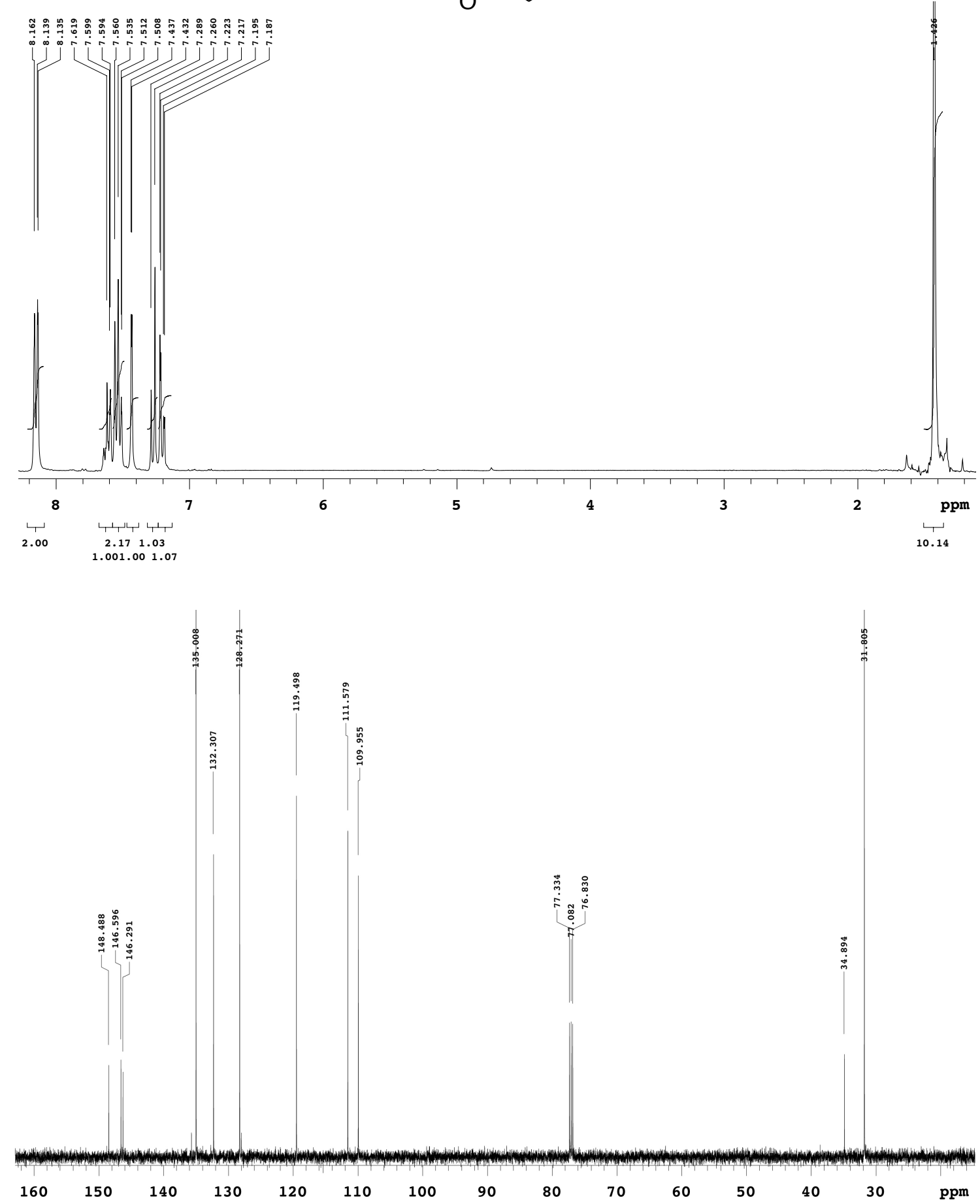


\section{1d}<smiles>CC(C)(C)c1ccc2c(c1)OB(c1ccc(F)cc1)O2</smiles>
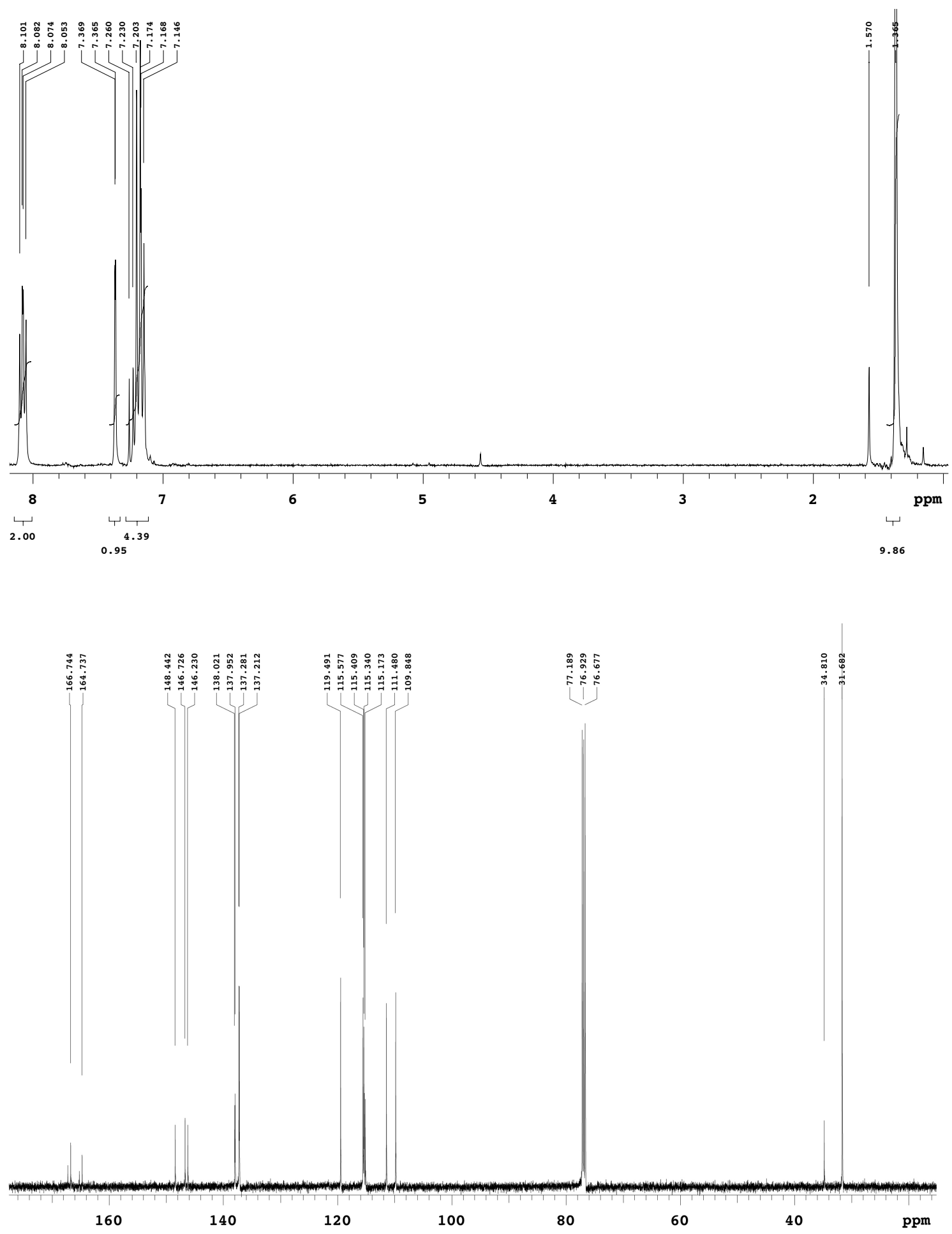

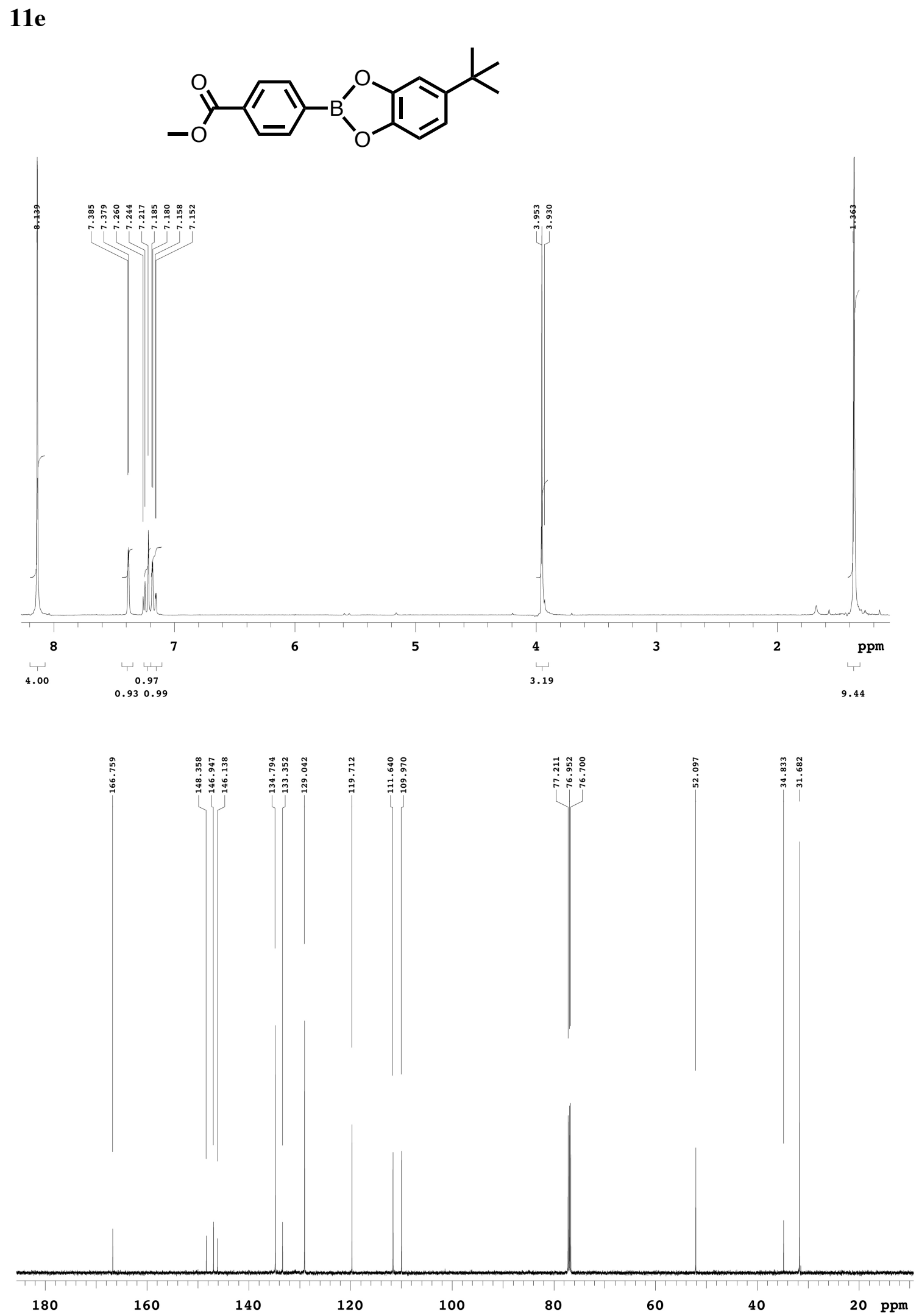
<smiles>CC(C)(C)c1ccc2c(c1)OB(c1ccc(C#N)cc1)O2</smiles>
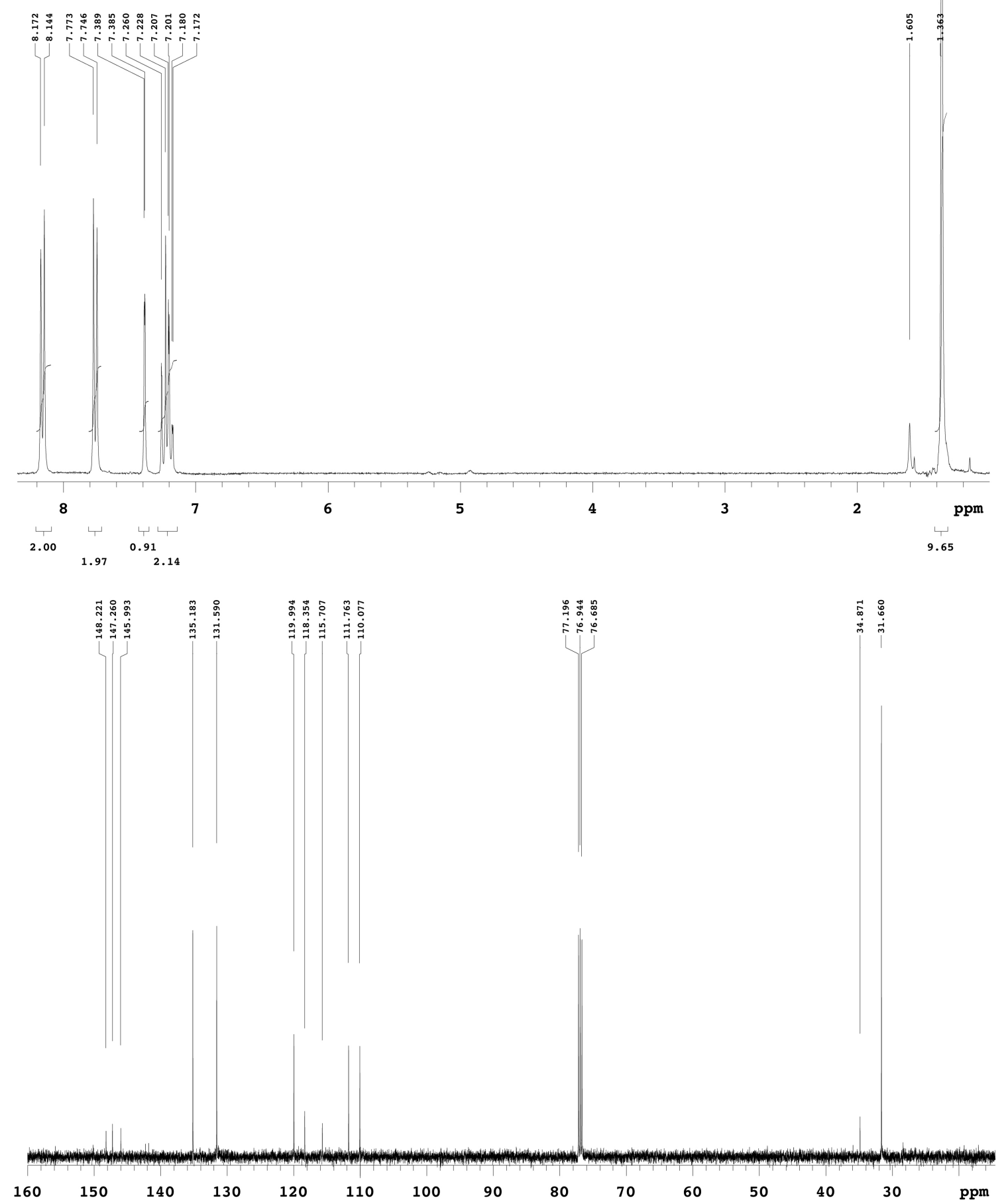
$12 a$<smiles>COc1ccc(B2Nc3ccc(C(C)(C)C)cc3N2)cc1</smiles>
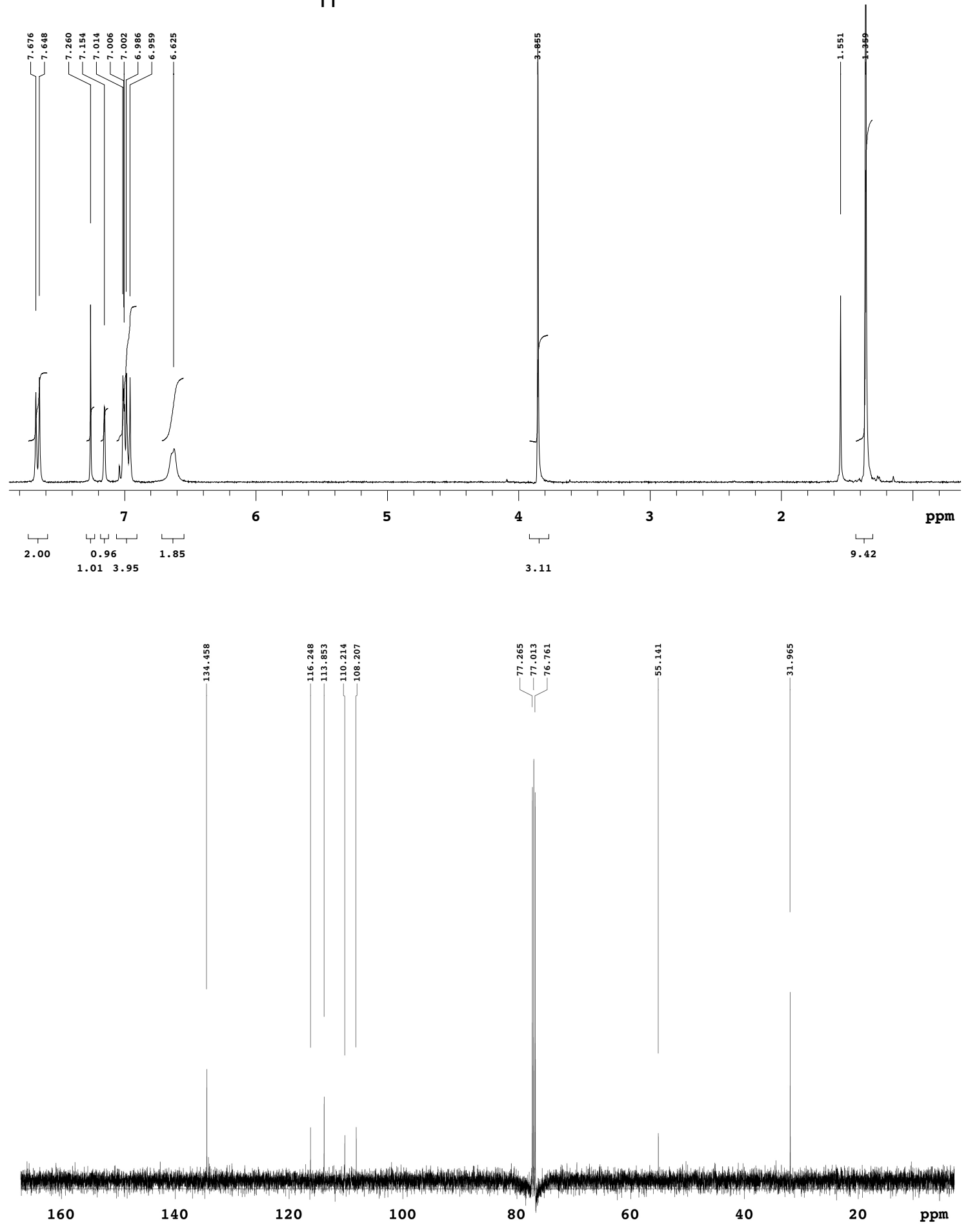
<smiles>CC(C)(C)c1ccc(B2Nc3ccc(C(C)(C)C)cc3N2)cc1</smiles>
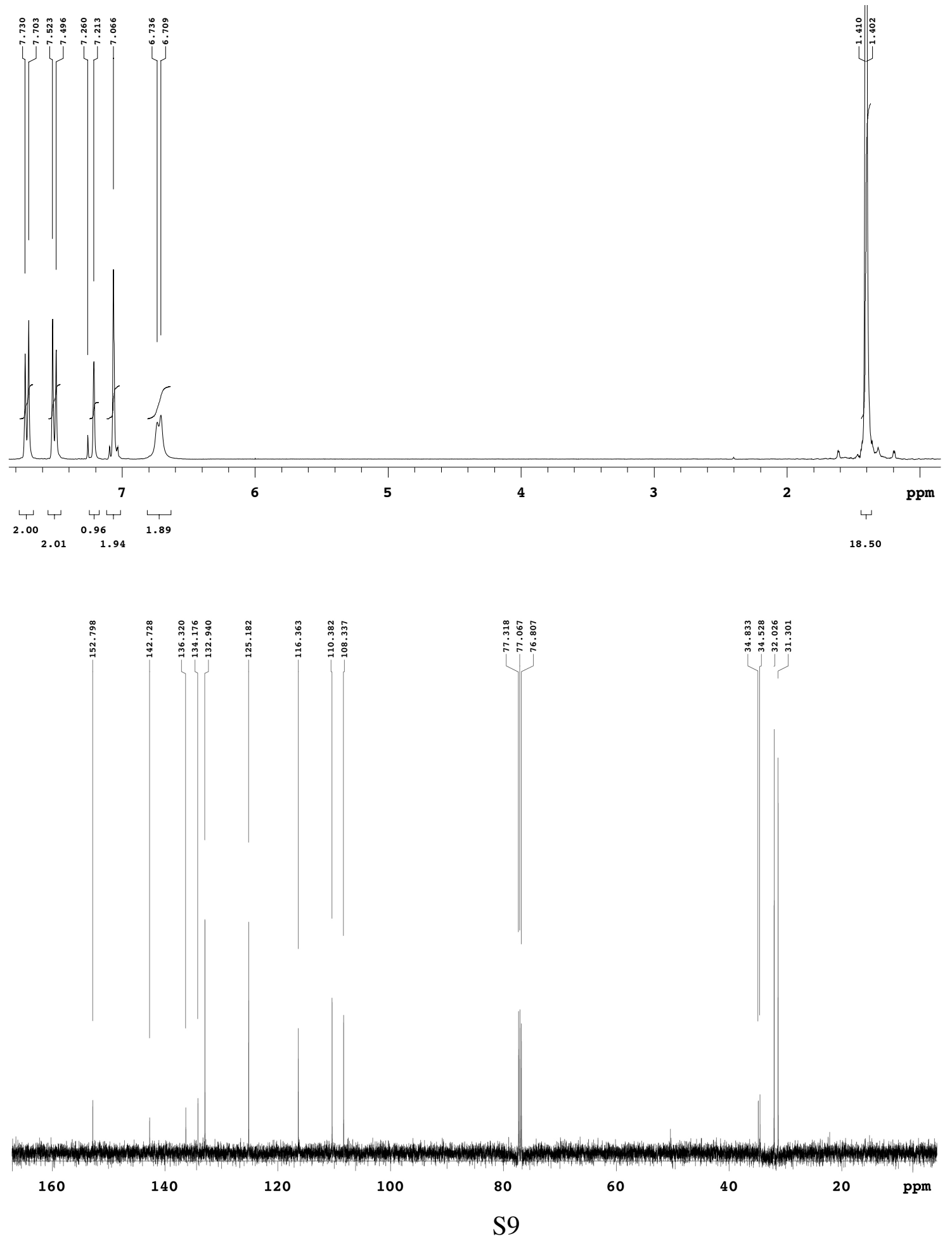
$12 c$<smiles>CC(C)(C)c1ccc2c(c1)NB(c1ccccc1)N2</smiles>
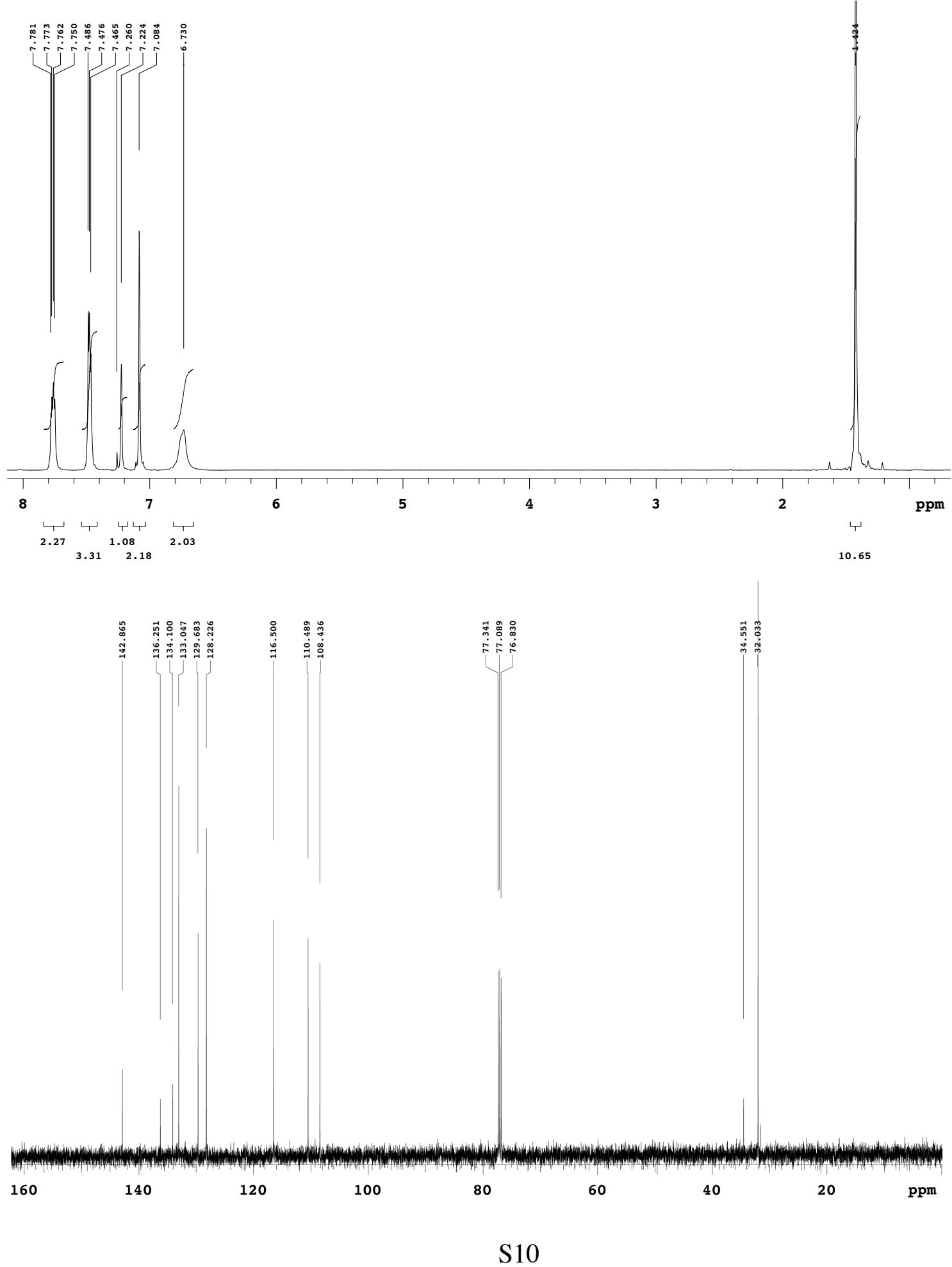
<smiles>CC(C)(C)c1ccc2c(c1)NB(c1ccc(F)cc1)N2</smiles>
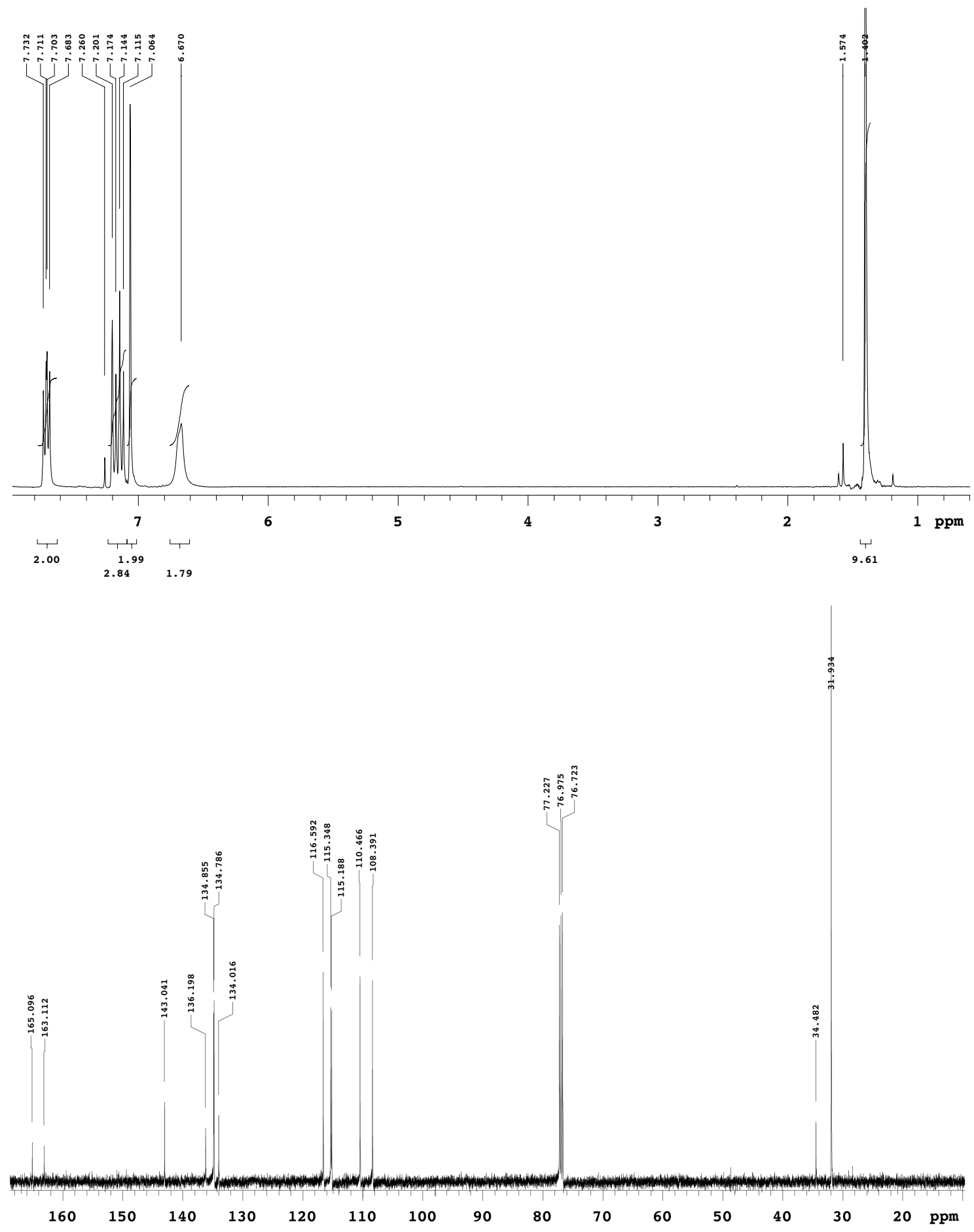
$12 \mathrm{e}$<smiles>COC(=O)c1ccc(B2Nc3ccc(C(C)(C)C)cc3N2)cc1</smiles>
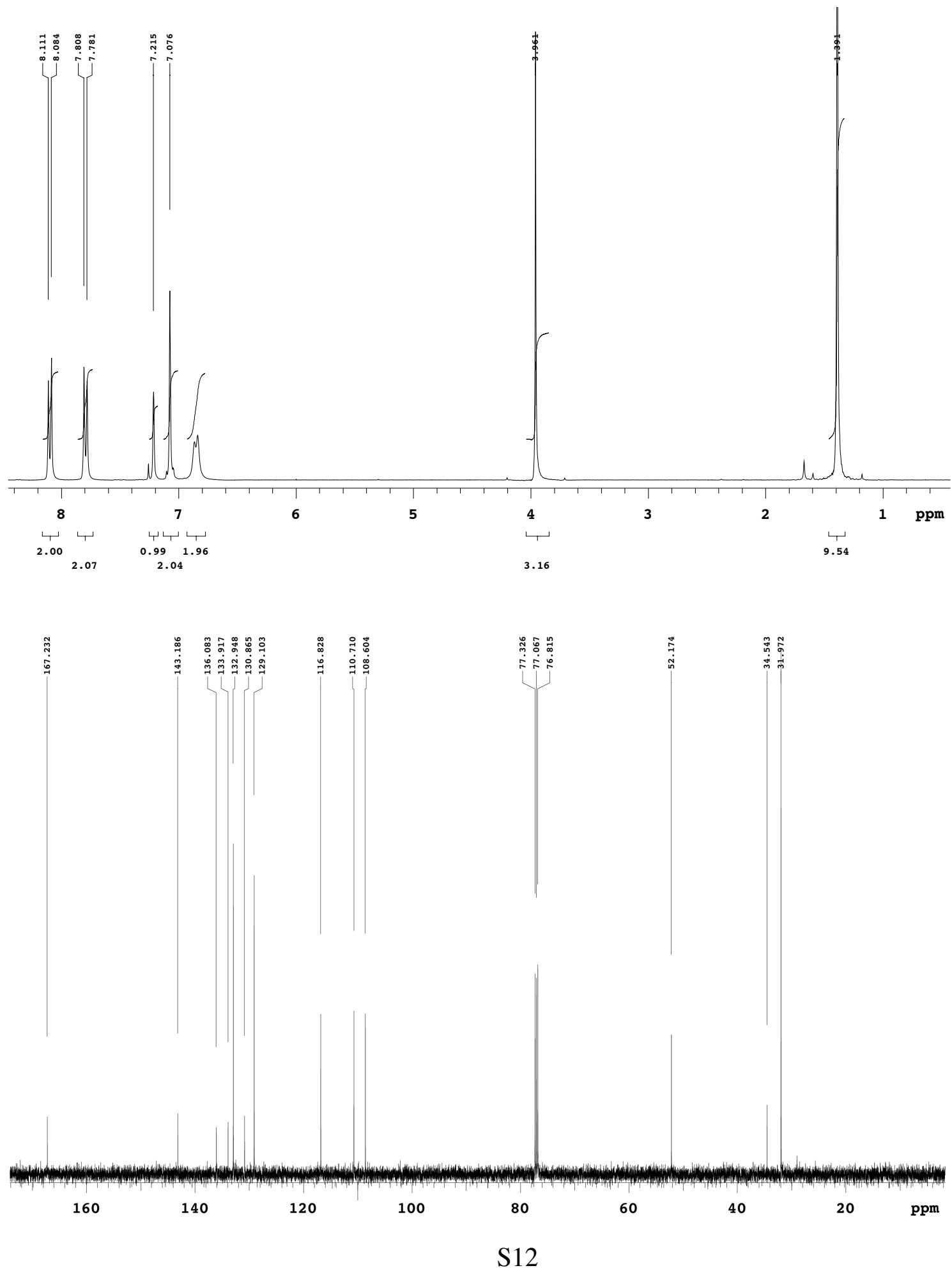
<smiles>CC(C)(C)c1ccc2c(c1)NB(c1ccc(C#N)cc1)N2</smiles>
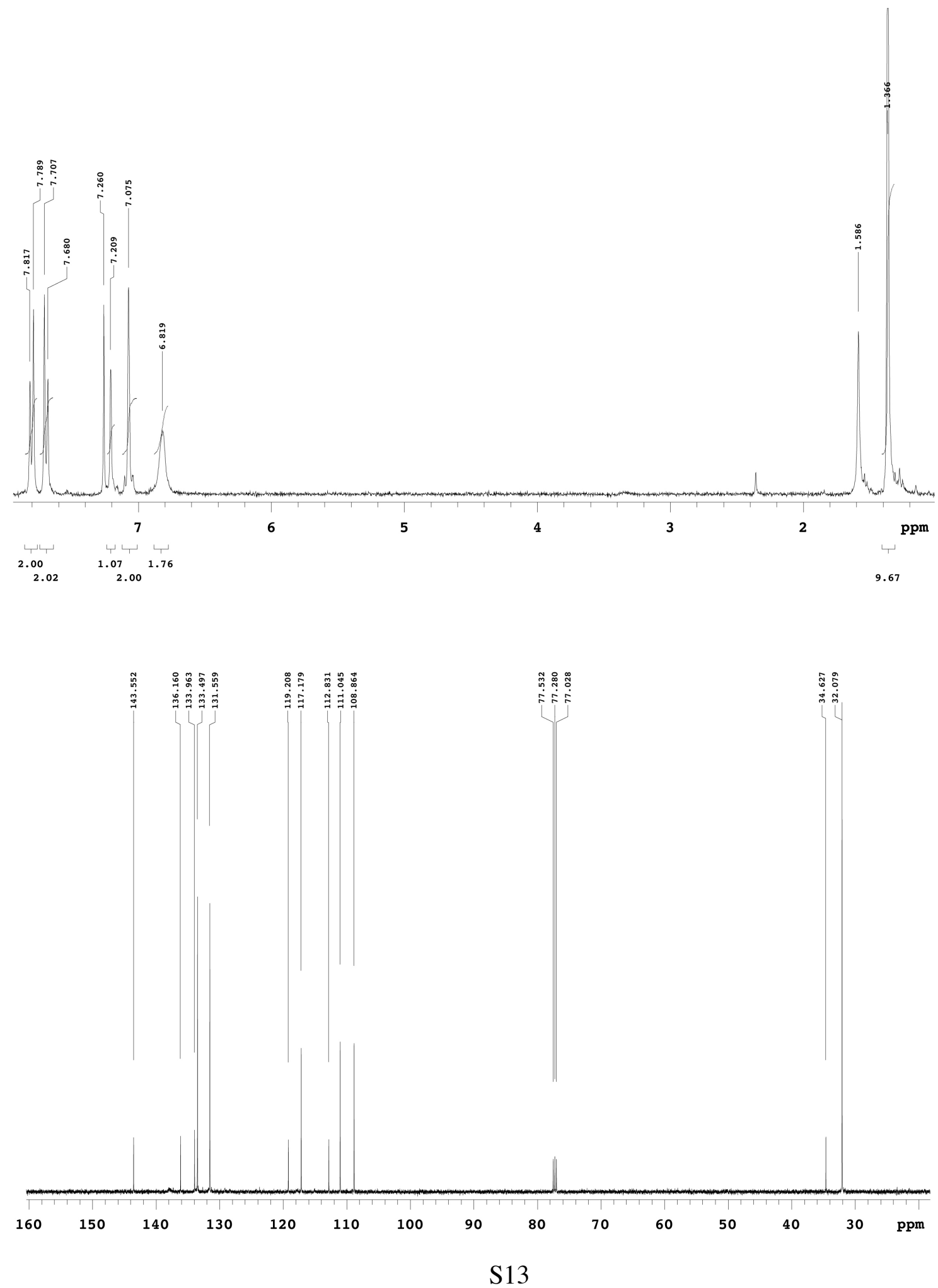
II. Analytical ${ }^{1} \mathbf{H}$ NMR Spectra of Boroxine Anydrides. The boroxine anhydride of each boronic acid (1-6) was prepared according to literature procedures. ${ }^{\text {1 }}$ These analytical samples were used to ensure correct labeling of any peaks characteristic to boroxine anhydride species that may be present in equilibrated ${ }^{1} \mathrm{H}$ NMR spectra. Furthermore, NMR-scale samples of each boroxine anhydride were then exposed to 2-3 drops of $\mathrm{D}_{2} \mathrm{O}$, which partially hydrolyzes each anhydride and allows for definitive characterization of ${ }^{1} \mathrm{H}$ NMR signals characteristic to each boronic acid (1-6).

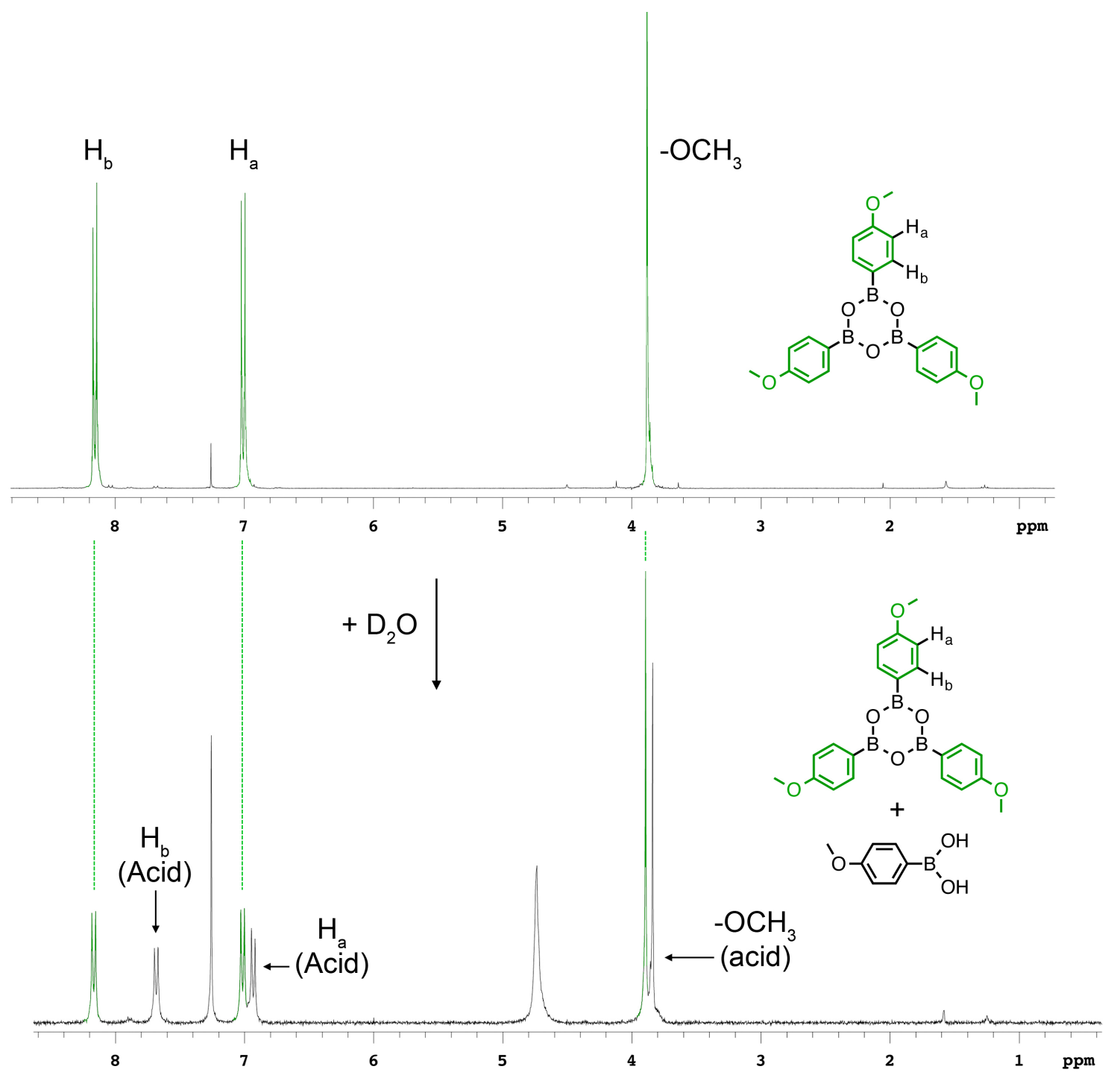

Figure S1. Top: ${ }^{1} \mathrm{H}$ NMR spectrum $\left(\mathrm{CDCl}_{3}, 300 \mathrm{MHz}, 298 \mathrm{~K}\right)$ of the boroxine anhydride of 4-methoxy boronic acid 1. Bottom: ${ }^{1} \mathrm{H}$ NMR spectrum of the same boroxine following the addition of 2-3 drops of $\mathrm{D}_{2} \mathrm{O}$, allowing for definitive characterization of hydrolyzed boronic acid signals versus boroxine anhydride signals. 


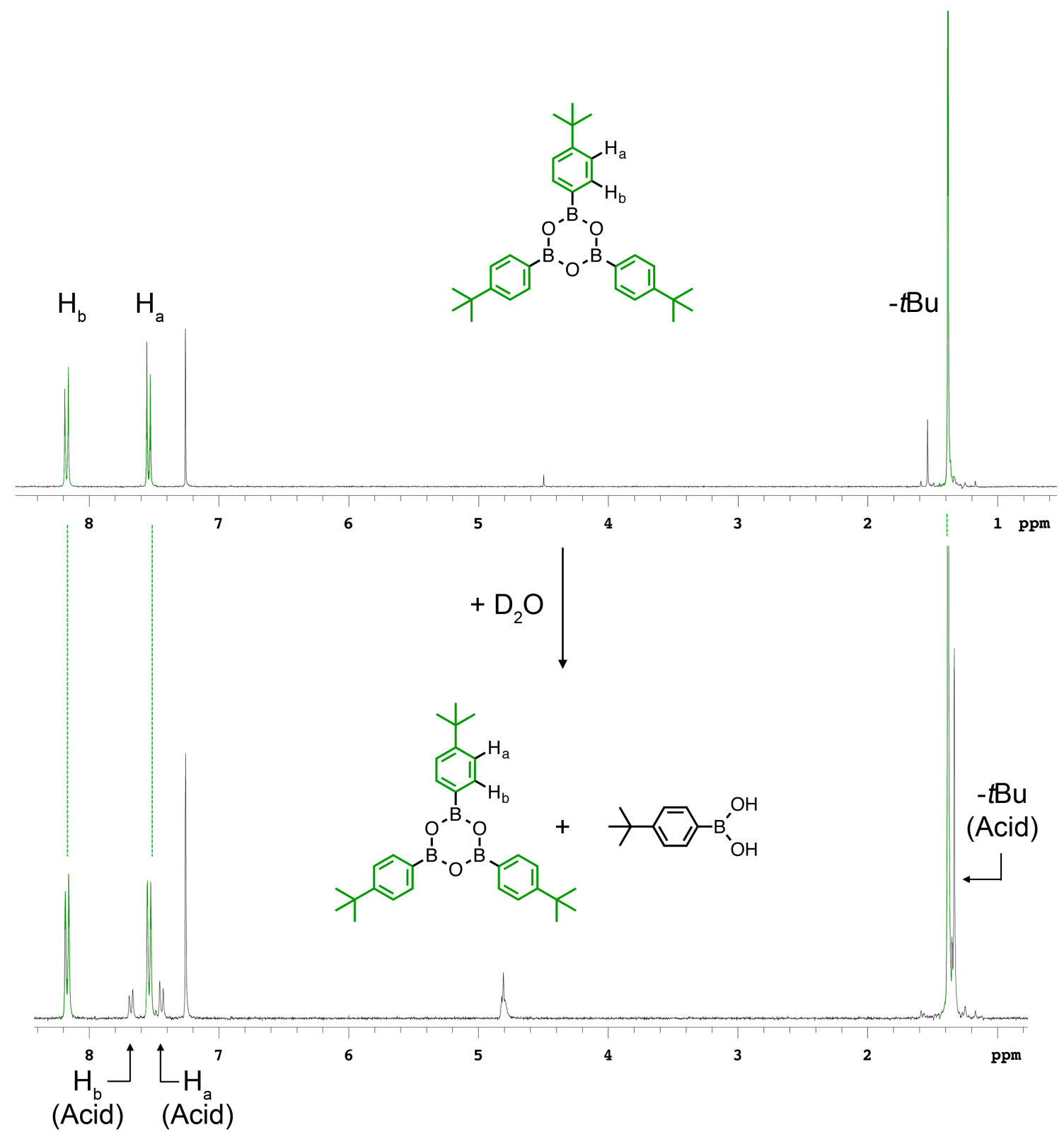

Figure S2. Top: ${ }^{1} \mathrm{H}$ NMR spectrum $\left(\mathrm{CDCl}_{3}, 300 \mathrm{MHz}, 298 \mathrm{~K}\right)$ of the boroxine anhydride of 4-tert-butyl boronic acid 2. Bottom: ${ }^{1} \mathrm{H}$ NMR spectrum of the same boroxine following the addition of 2-3 drops of $\mathrm{D}_{2} \mathrm{O}$, allowing for definitive characterization of hydrolyzed boronic acid signals versus boroxine anhydride signals. 


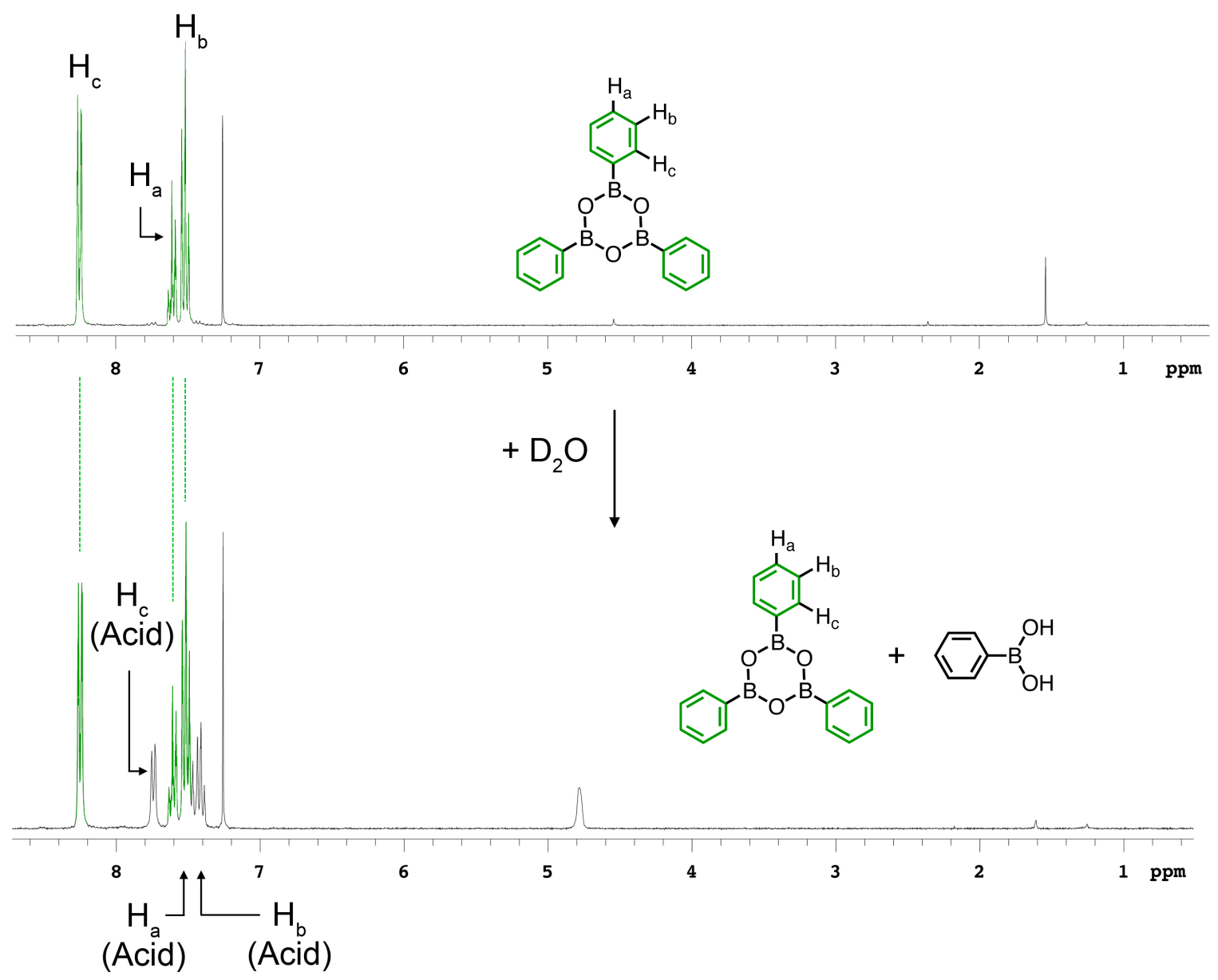

Figure S3. Top: ${ }^{1} \mathrm{H} \mathrm{NMR}$ spectrum $\left(\mathrm{CDCl}_{3}, 300 \mathrm{MHz}, 298 \mathrm{~K}\right)$ of the boroxine anhydride of phenyl boronic acid 3. Bottom: ${ }^{1} \mathrm{H}$ NMR spectrum of the same boroxine following the addition of 2-3 drops of $\mathrm{D}_{2} \mathrm{O}$. Signals for boroxine anhydride and boronic acid species, particularly $\mathrm{H}_{\mathrm{a}}$ and $\mathrm{H}_{\mathrm{b}}$ of each, overlap in the region between 7.64-7.39 ppm. This overlap makes it more difficult to definitively assign all signals of the boroxine versus hydrolyzed boronic acid. For this reason the determination of relative percentages of assembled boronate ester 11c relies more upon the more clearly separated signals of the tert-butyl moiety of catechol derivative 7 (see Figure S9 and Figures S19-S22). 


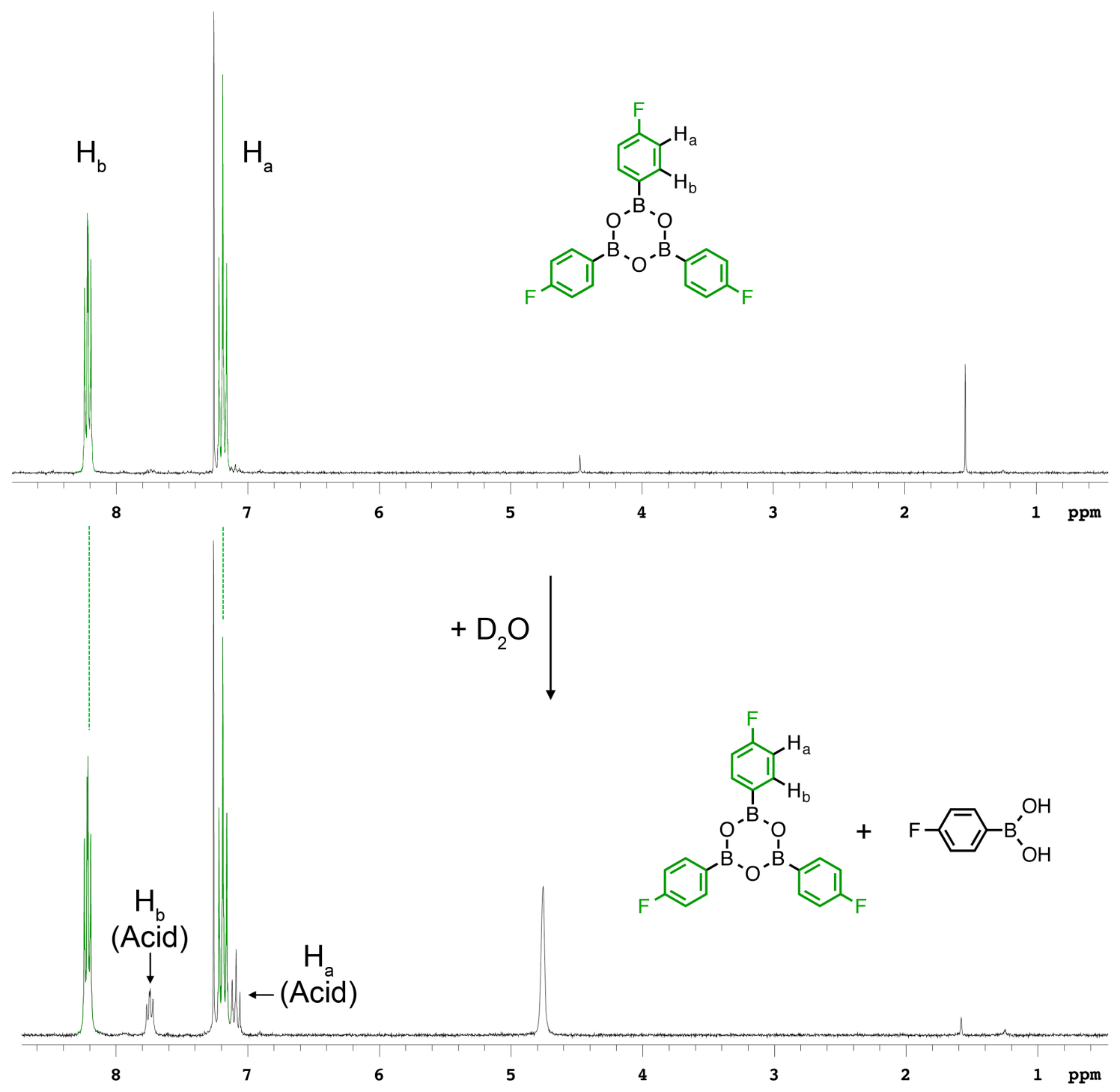

Figure S4. Top: ${ }^{1} \mathrm{H}$ NMR spectrum $\left(\mathrm{CDCl}_{3}, 300 \mathrm{MHz}, 298 \mathrm{~K}\right)$ of the boroxine anhydride of 4-fluoro boronic acid 4. Bottom: ${ }^{1} \mathrm{H}$ NMR spectrum of the same boroxine following the addition of 2-3 drops of $\mathrm{D}_{2} \mathrm{O}$, allowing for definitive characterization of hydrolyzed boronic acid signals versus boroxine anhydride signals. 


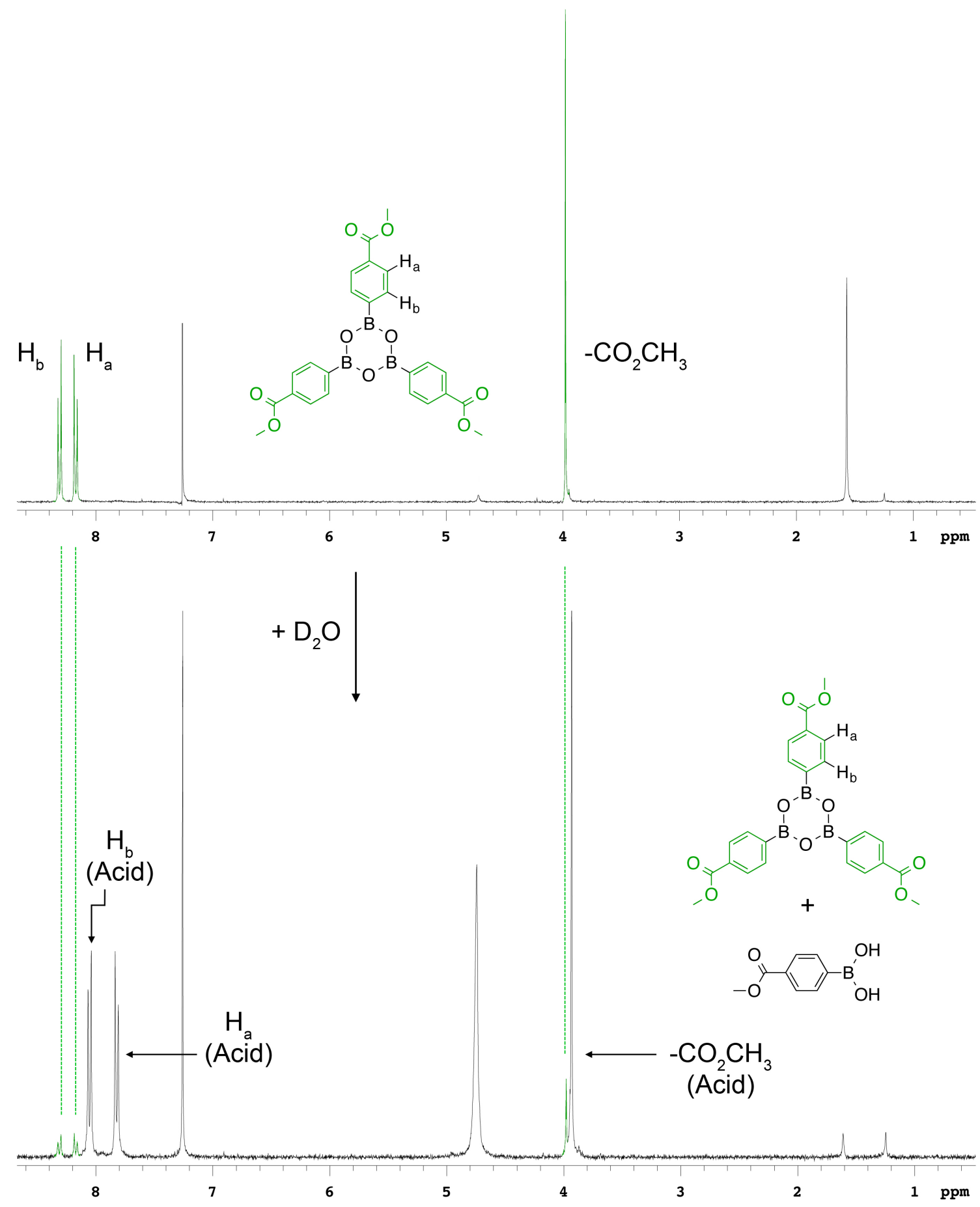

Figure S5. Top: ${ }^{1} \mathrm{H}$ NMR spectrum $\left(\mathrm{CDCl}_{3}, 300 \mathrm{MHz}, 298 \mathrm{~K}\right)$ of the boroxine anhydride of 4-carboxymethylcarbonyl boronic acid 5. Bottom: ${ }^{1} \mathrm{H}$ NMR spectrum of the same boroxine following the addition of 2-3 drops of $\mathrm{D}_{2} \mathrm{O}$, allowing for definitive characterization of hydrolyzed boronic acid signals versus boroxine anhydride signals. 


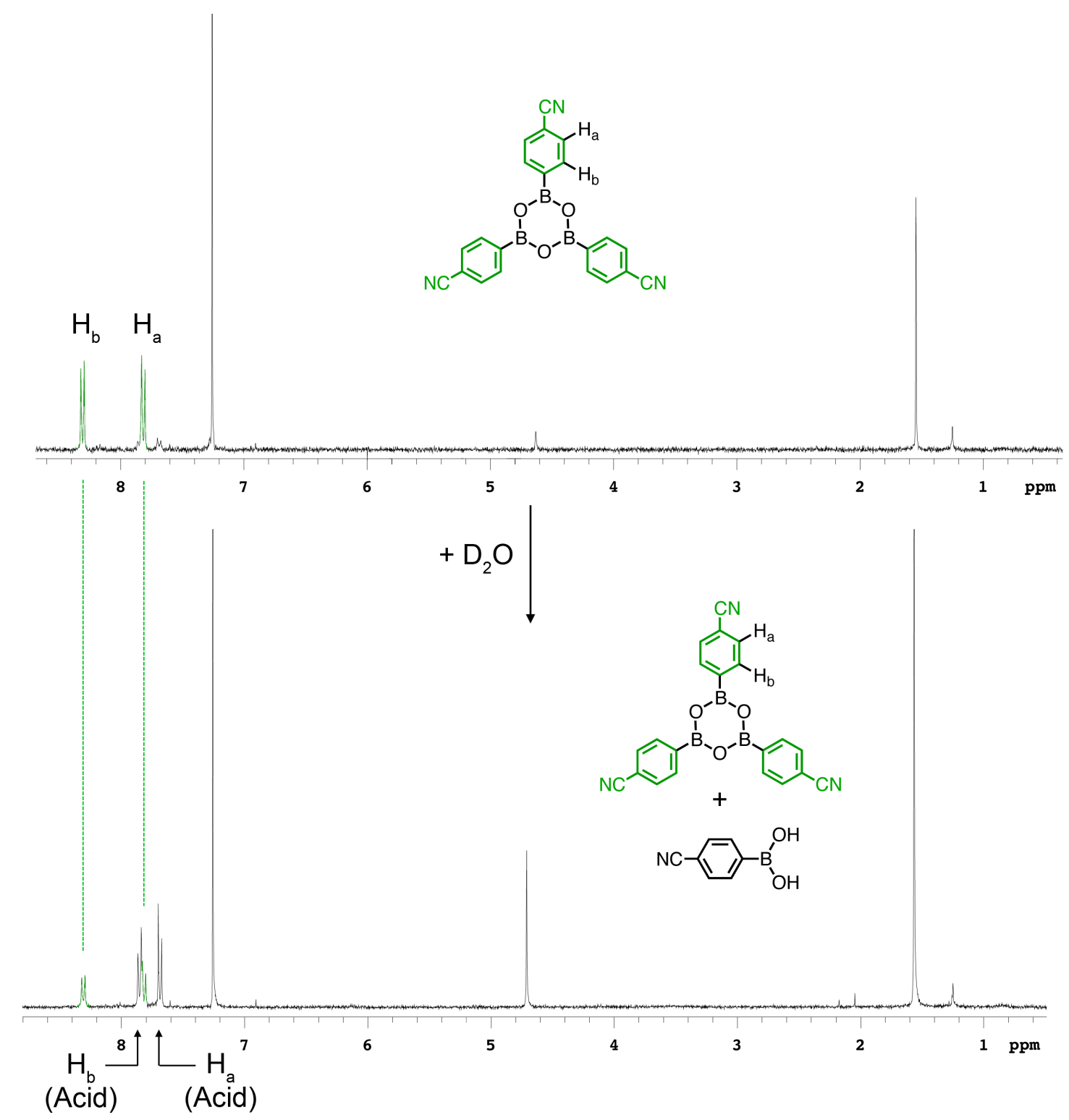

Figure S6. Top: ${ }^{1} \mathrm{H}$ NMR spectrum $\left(\mathrm{CDCl}_{3}, 300 \mathrm{MHz}, 298 \mathrm{~K}\right)$ of the boroxine anhydride of 4-cyano boronic acid 6. Bottom: ${ }^{1} \mathrm{H}$ NMR spectrum of the same boroxine following the addition of 2-3 drops of $\mathrm{D}_{2} \mathrm{O}$. Note that small but observable signals corresponding to the 4-cyano phenylboronic acid can be seen even in the top spectrum of the boroxine (particularly $\mathrm{H}_{\mathrm{a}}$ of the acid). It is likely that trace moisture in the $\mathrm{CDCl}_{3}$ promotes partial hydrolysis of the anhydride. Still, the above spectra allow for definitive characterization of hydrolyzed boronic acid signals versus boroxine anhydride signals. 
III. ${ }^{1} \mathrm{H}$ NMR Spectra of Equilibrated Boronate Esters. Equimolar solutions of each boronic acid and each donor were mixed in dry $\mathrm{CDCl}_{3}(0.05 \mathrm{M})$, allowed to reach equilibrium, and used to determine percentages of assembled vs unassembled species.

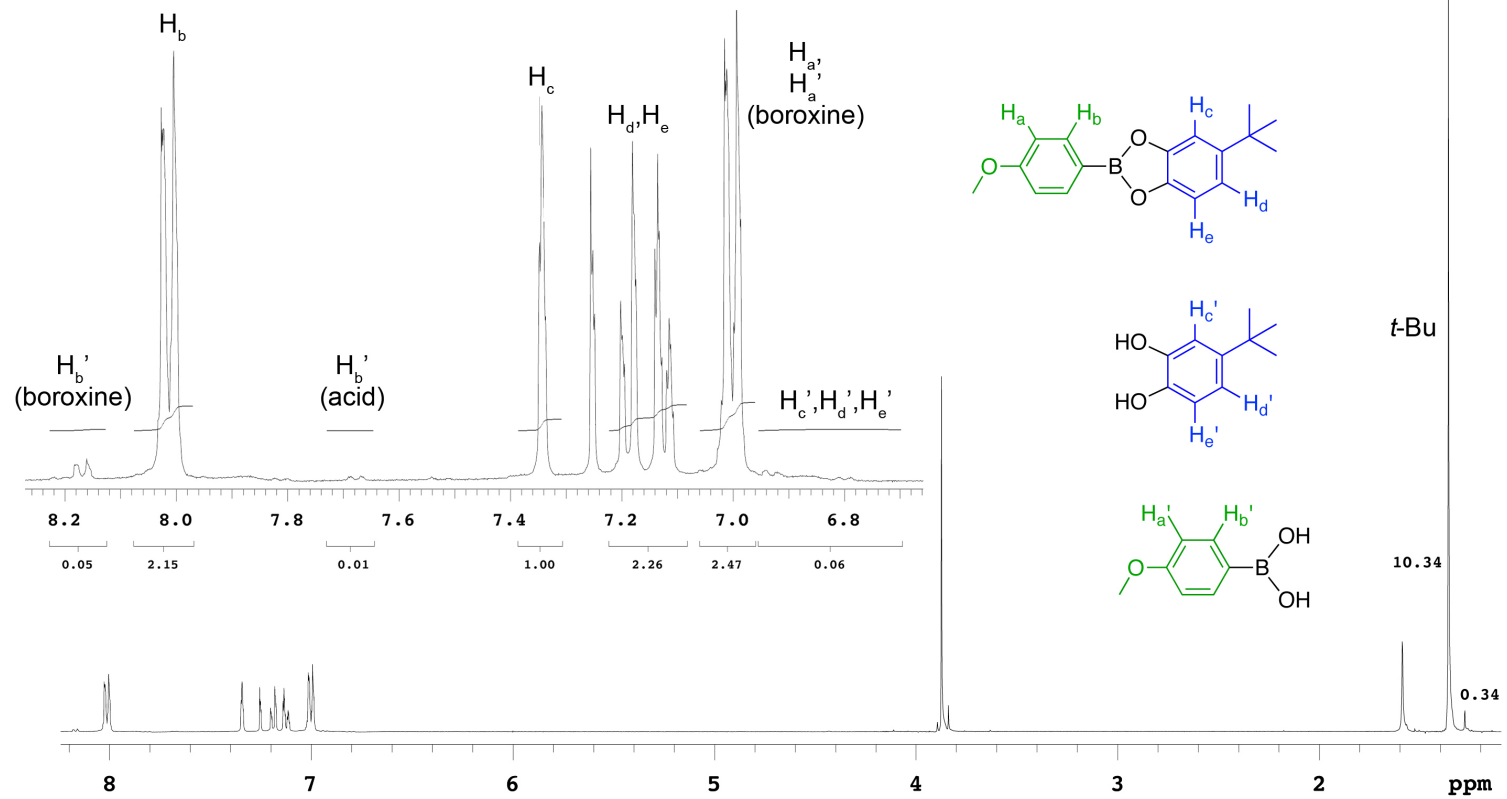

Figure S7. ${ }^{1} \mathrm{H}$ NMR spectrum of an equilibrated mixture of 4-methoxyphenylboronic acid (1) and 4-tert-butylcatechol (7) to give 11a. Percent ester calculation based primarily on $t$-butyl peaks: $10.34 /(10.34+0.34)=96.8 \%$. Calculation from $\mathrm{H}_{\mathrm{b}}$ of the ester, boroxine, and acid gives: $2.15 /(2.15+0.05 / 3+0.01)=98.8 \%$ however low signal-to-noise for the boroxine and acid peaks makes this assessment less reliable.
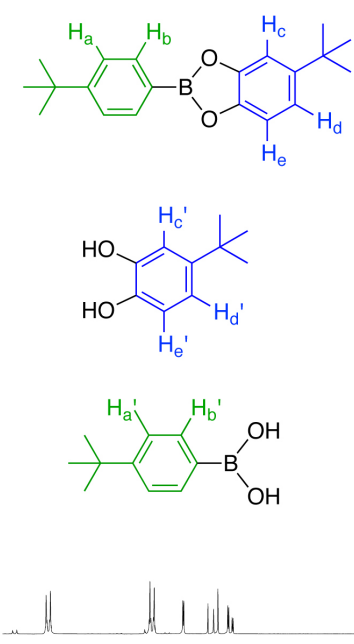

8

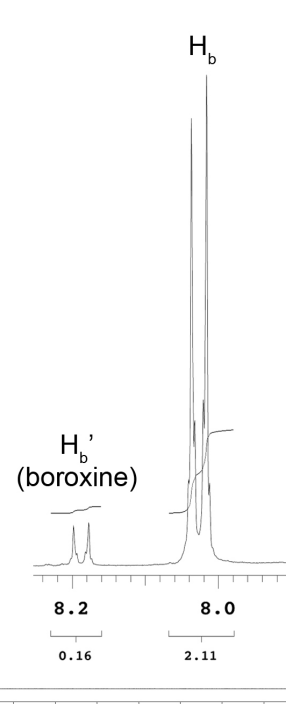

6

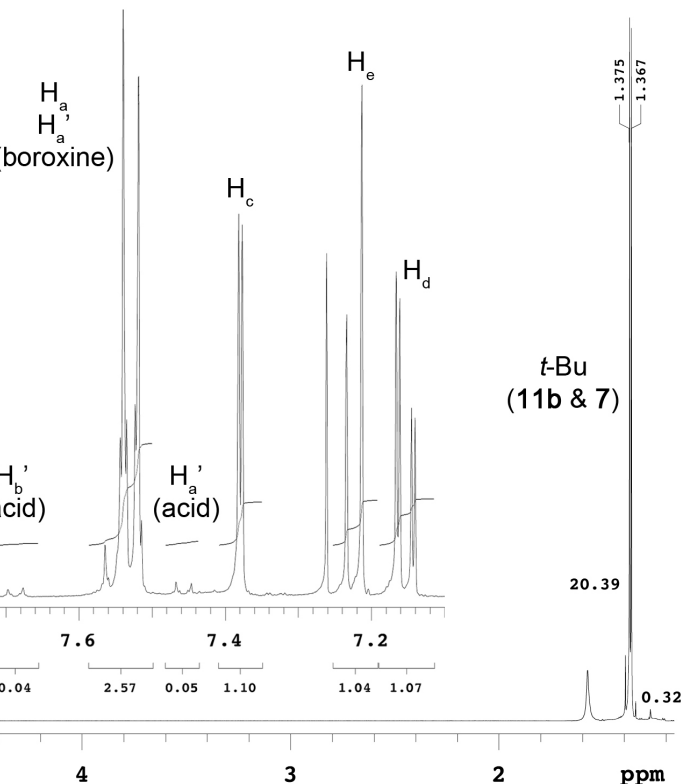

5

3

ppm

Figure S8. ${ }^{1} \mathrm{H}$ NMR spectrum of an equilibrated mixture of 4-tert-butylphenylboronic acid (2) and 4-tert-butylcatechol (7) to give 11b. Percent ester calculation based on $\mathrm{H}_{\mathrm{b}}$ of the ester, boroxine, and acid: $2.11 /(2.11+0.16 / 3+0.04)=95.8 \%$. Overlap of signals in the $t$-butyl region prevented its use for calculating the percent of ester formation. 

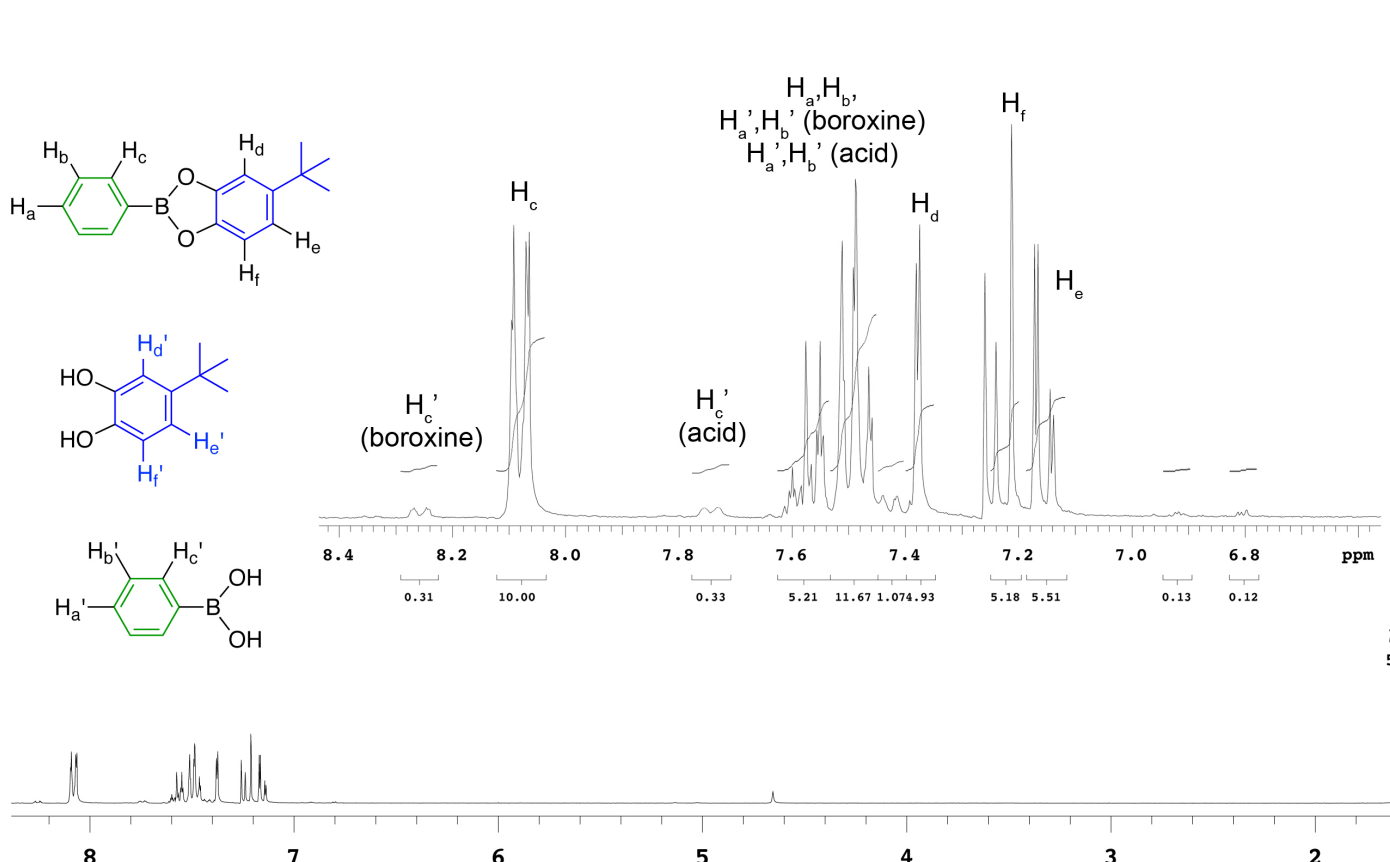

Figure S9. ${ }^{1} \mathrm{H}$ NMR spectrum of an equilibrated mixture of phenylboronic acid (3) and 4tert-butylcatechol (7) to give approximately $97.5 \%$ of ester product 11c. Percent ester calculation based primarily on $t$-butyl peaks: $51.56 /(51.56+1.31)=97.5 \%$. Calculation from $\mathrm{H}_{\mathrm{c}}$ of the ester, boroxine, and acid gives: $10.0 /(10.0+0.31 / 3+0.33)=95.8 \%$ however low signal-to-noise for the boroxine and boronic acid peaks makes this assessment less reliable.
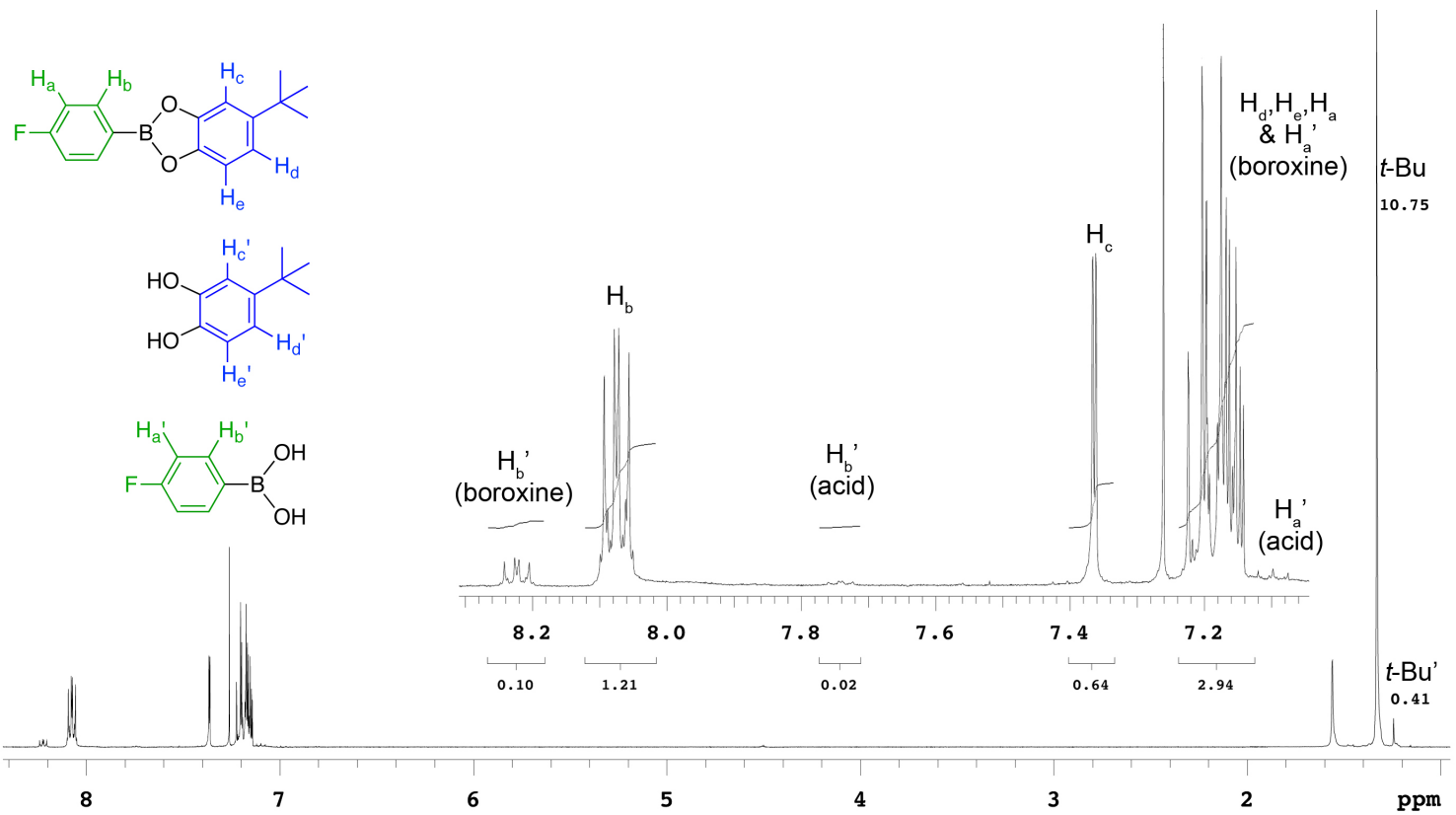

Figure S10. ${ }^{1} \mathrm{H}$ NMR spectrum of an equilibrated mixture of 4-fluorophenylboronic acid (4) and 4-tert-butylcatechol (7) to give ester product 11d. Percent ester calculation based primarily on $t$-butyl peaks: $10.75 /(10.75+0.41)=96.3 \%$. Calculation from $\mathrm{H}_{\mathrm{b}}$ of the ester, boroxine, and acid gives: $1.21 /(1.21+0.10 / 3+0.02)=95.8 \%$ however low signal-tonoise for the boroxine and acid peaks makes this assessment less reliable. 


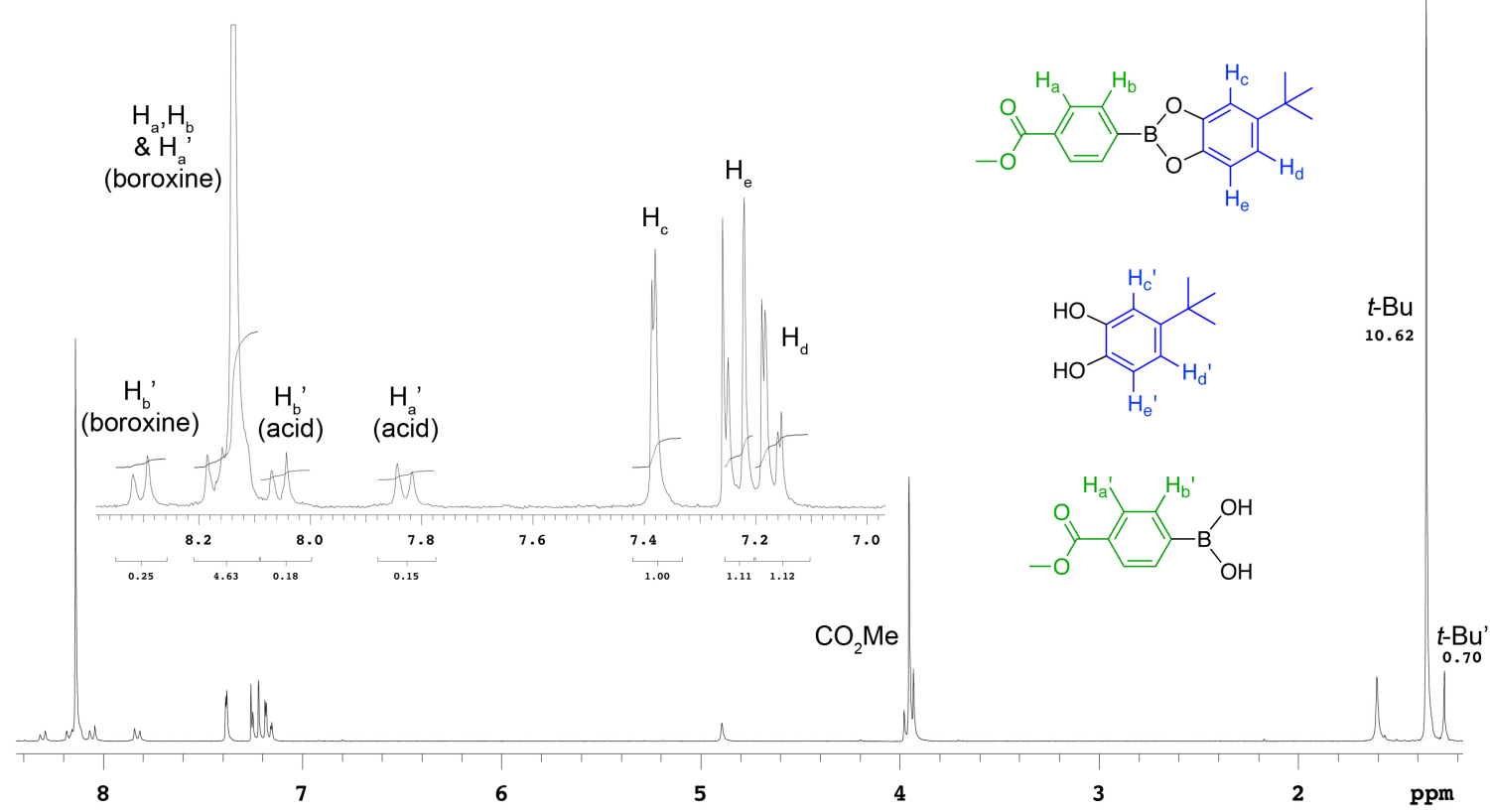

Figure S11. ${ }^{1} \mathrm{H}$ NMR spectrum of an equilibrated mixture of 4-methoxycarbonyl phenylboronic acid (5) and 4-tert-butylcatechol (7) to give ester product 11e. Percent ester calculation based on $t$-butyl peaks: $10.62 /(10.62+0.70)=93.8 \%$. Overlap of signals of related boroxine and acid species in the aromatic region prohibits their use as a reliable means of calculating the amount of ester formation.

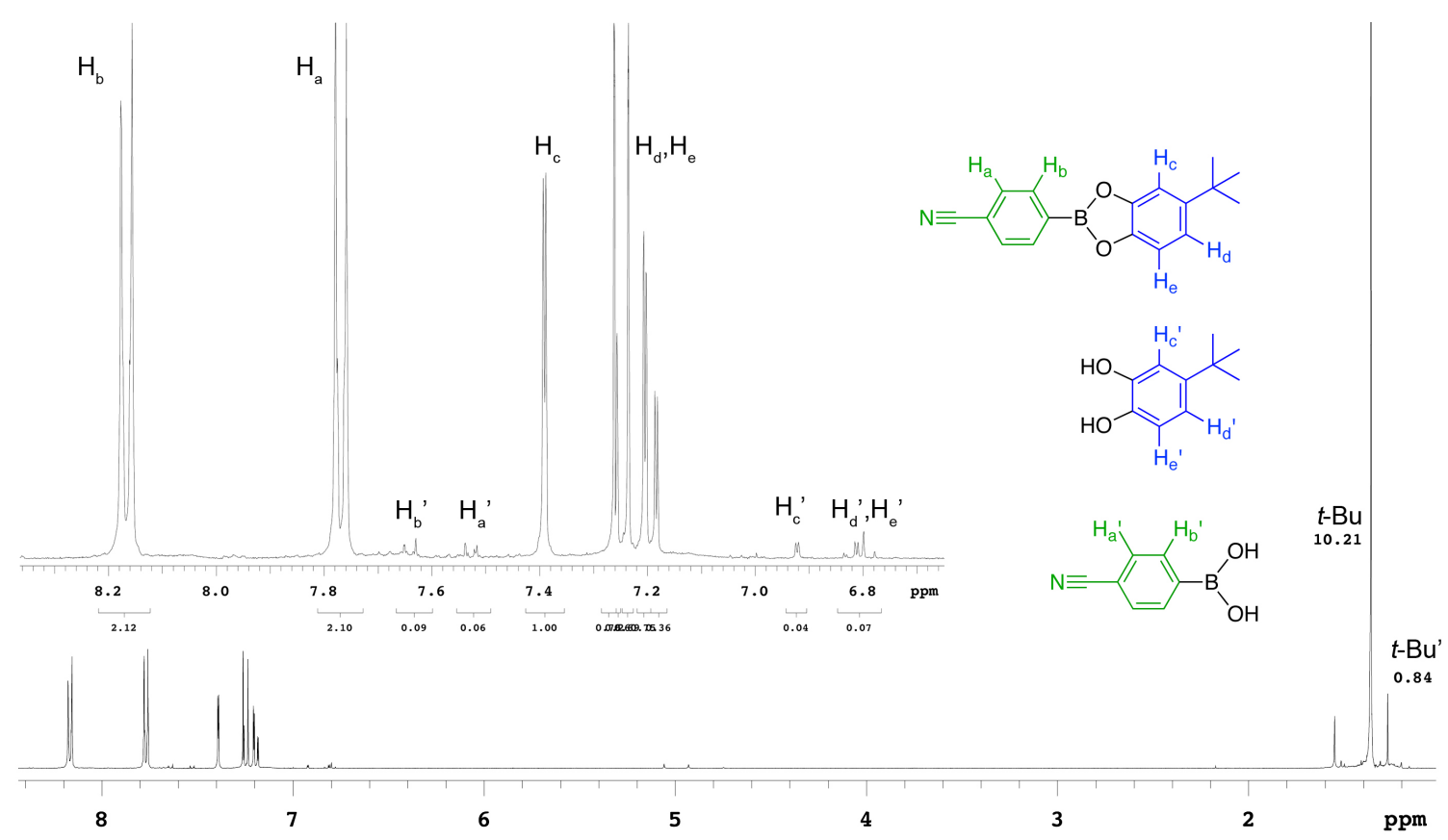

Figure S12. ${ }^{1} \mathrm{H}$ NMR spectrum of an equilibrated mixture of 4-cyanophenylboronic acid (6) and 4-tert-butylcatechol (7) to give ester product 11f. Percent ester calculation based on $t$-butyl peaks: $10.21 /(10.21+0.84)=92.4 \%$. Calculation from $\mathrm{H}_{\mathrm{b}}$ of the ester and acid signals gives: $2.12 /(2.12+0.09)=95.9 \%$ however very low signal-to-noise for the acid peak $\mathrm{H}_{\mathrm{b}}$ makes this assessment less reliable. 


\section{IV. ${ }^{1}$ H NMR Spectra of Equilibrated Diazaboroles}

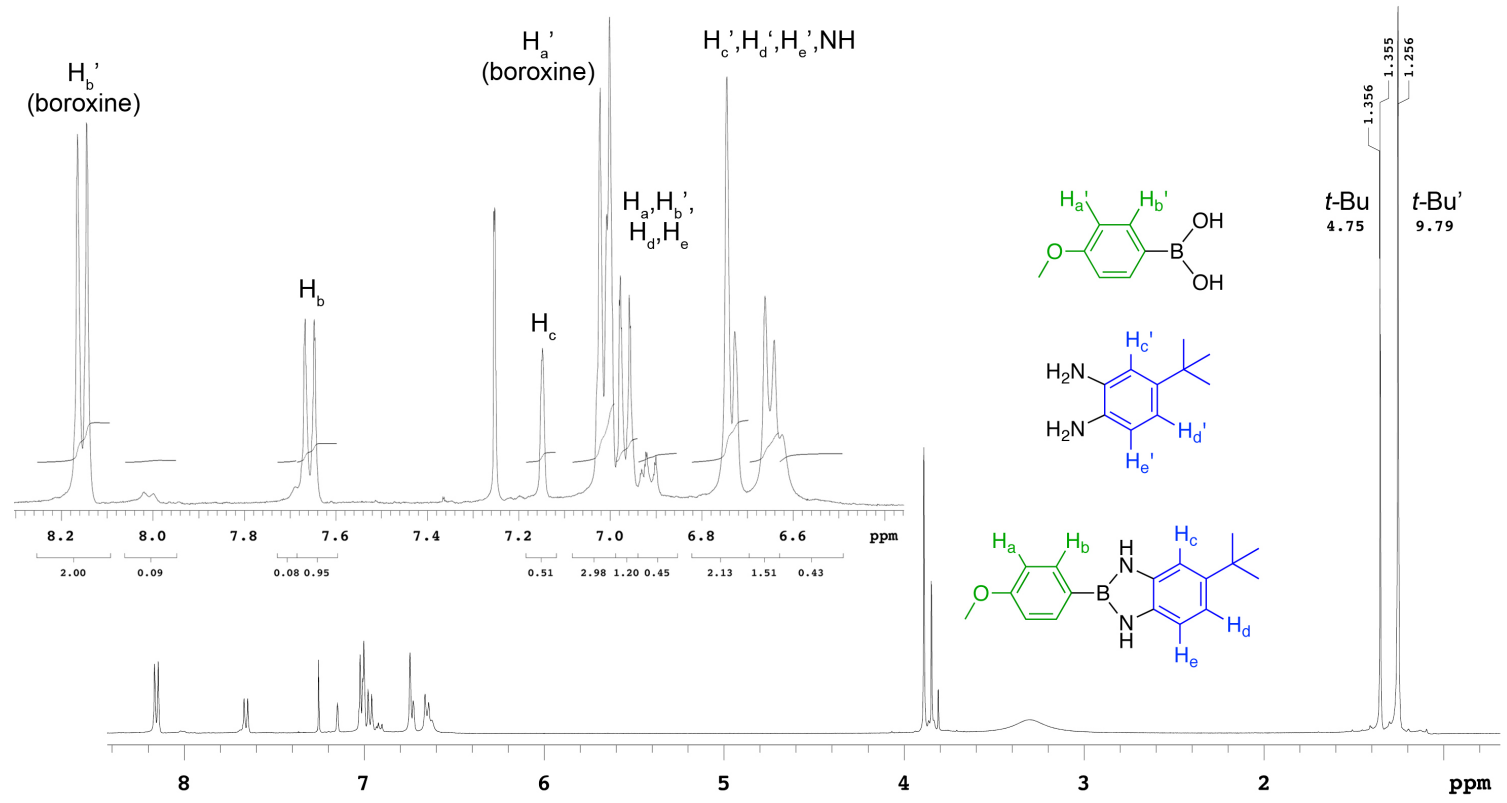

Figure S13. ${ }^{1} \mathrm{H}$ NMR spectrum of an equilibrated mixture of 4-methoxyphenylboronic acid (1) and 4-tert-butyl-ortho-phenylenediamine (8) to give diazaborole product 12a. Percent diazaborole calculation based primarily on $t$-butyl peaks: $4.75 /(4.75+9.79)=$ $32.7 \%$. Peak overlap and low signal-to-noise make the percent product calculation based on aromatic signals less reliable.

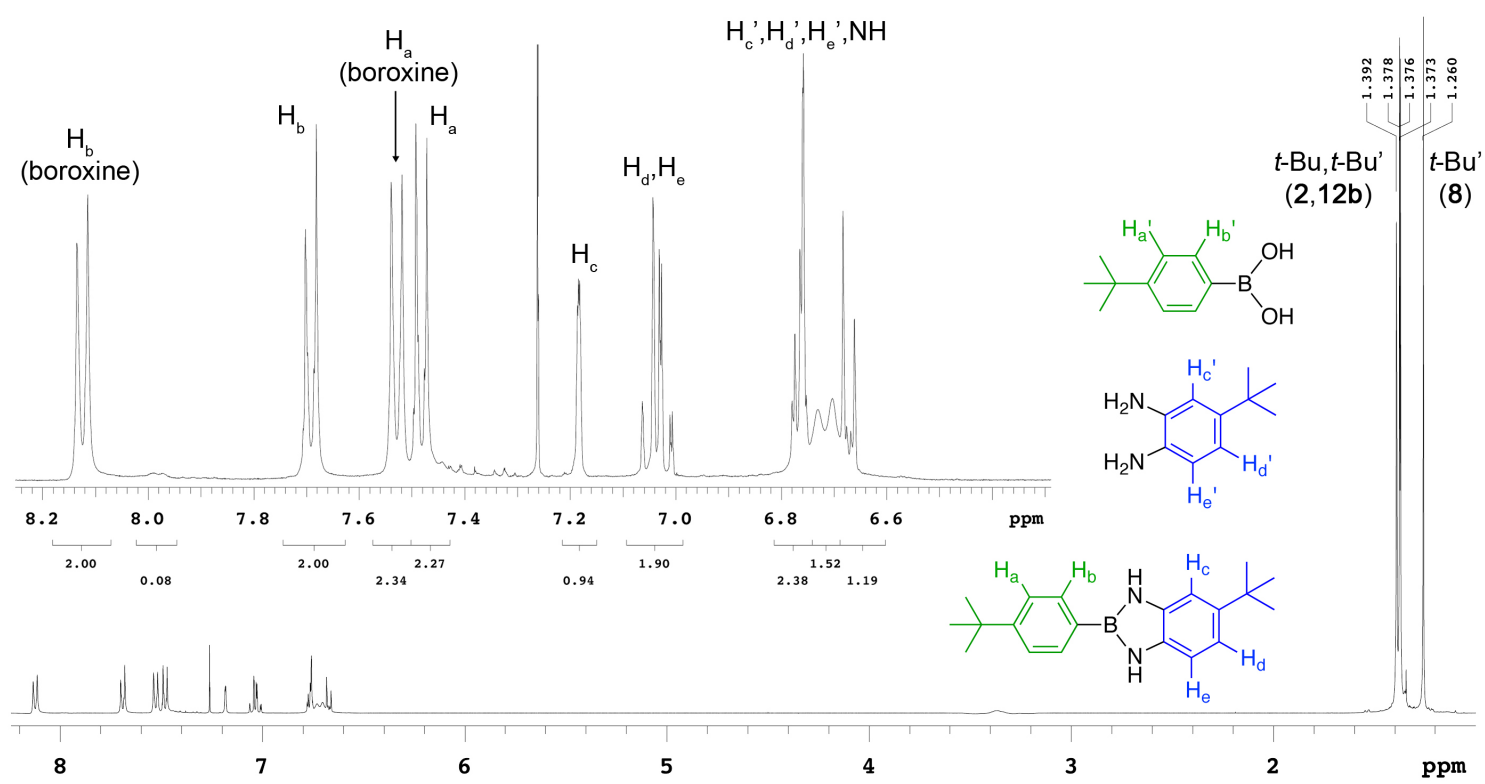

Figure S14. ${ }^{1} \mathrm{H}$ NMR spectrum of an equilibrated mixture of 4-tert-butylphenylboronic acid (2) and 4-tert-butyl-ortho-phenylenediamine (8) to give diazaborole product $\mathbf{1 2 b}$. Due to overlap of $t$-butyl signals, the percent diazaborole formation was calculated using the integration of signals corresponding to $\mathrm{H}_{\mathrm{c}-\mathrm{e}}$ of the diazaborole $\mathrm{vs}_{\mathrm{c}^{\prime}-\mathrm{e}}$, of diamine $\mathbf{8}$ : $(0.94+1.90) /(2.38+1.19)=44.3 \%$. 


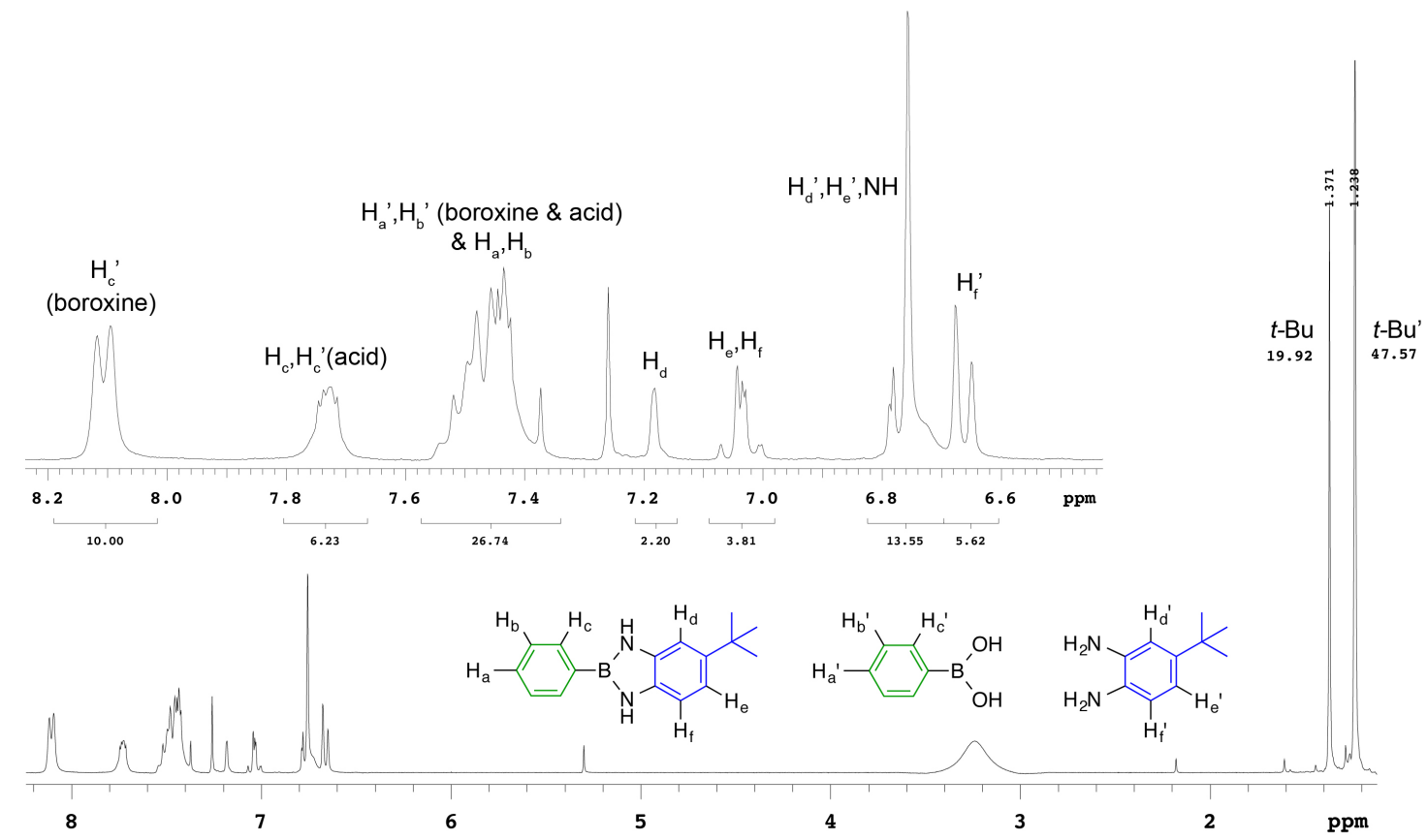

Figure S15. ${ }^{1} \mathrm{H}$ NMR spectrum of an equilibrated mixture of phenylboronic acid (3) and 4-tert-butyl-ortho-phenylenediamine (8) to give diazaborole product 12c. Percent diazaborole calculation based on $t$-butyl peaks: $19.92 /(19.92+47.57)=29.5 \%$. Significant overlap of aromatic signals makes calculating the percent formation based on aromatic peaks less reliable.

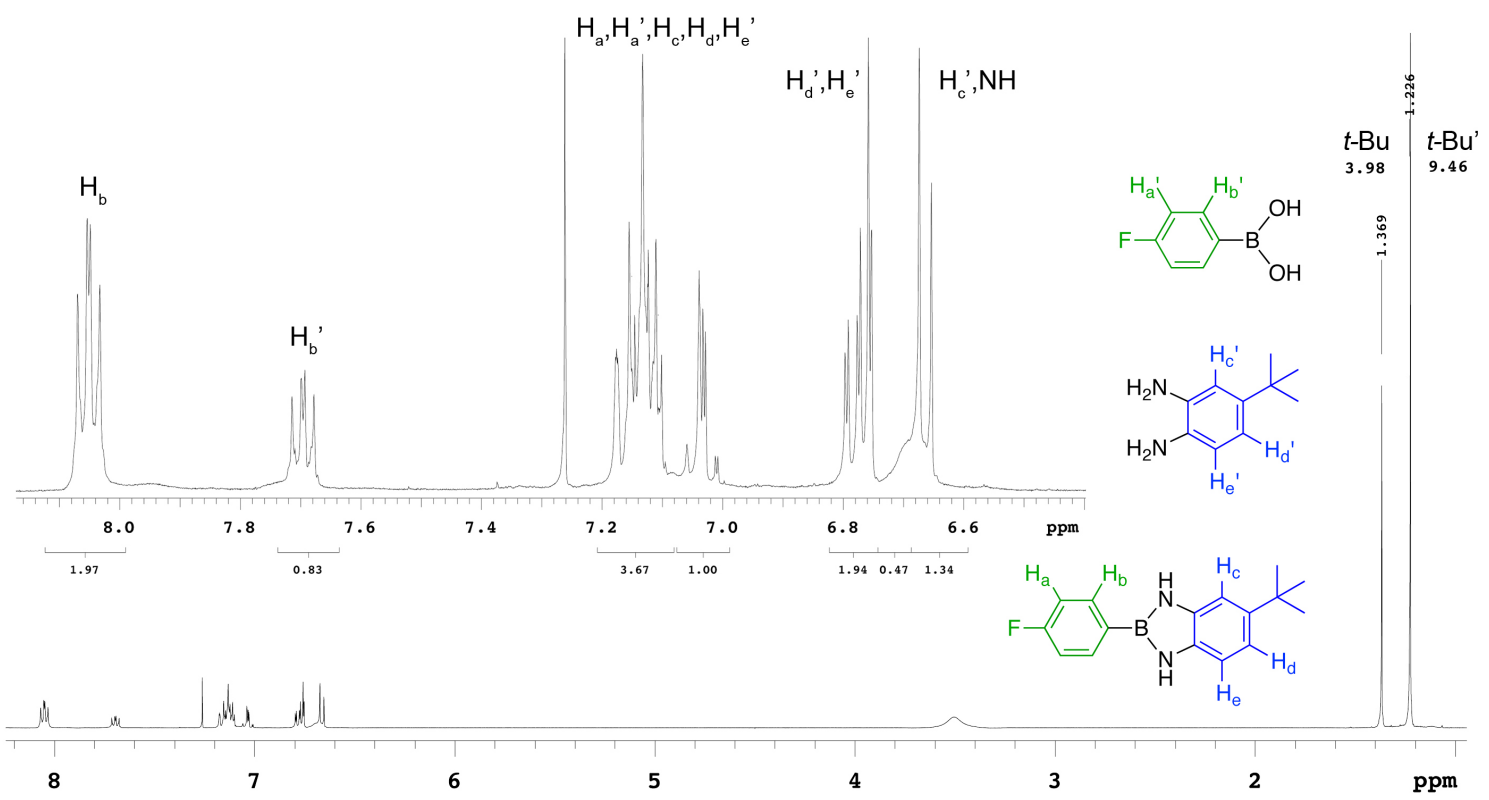

Figure S16. ${ }^{1} \mathrm{H}$ NMR spectrum of an equilibrated mixture of 4-fluorophenylboronic acid (4) and 4-tert-butyl-ortho-phenylenediamine (8) to give diazaborole product 12d. Percent diazaborole calculation based primarily on $t$-butyl peaks: $3.98 /(3.98+9.46)=29.6 \%$. Calculation from $\mathrm{H}_{\mathrm{b}}$ of the diazaborole and acid also gives: $0.83 /(0.83+1.97)=29.6 \%$. 


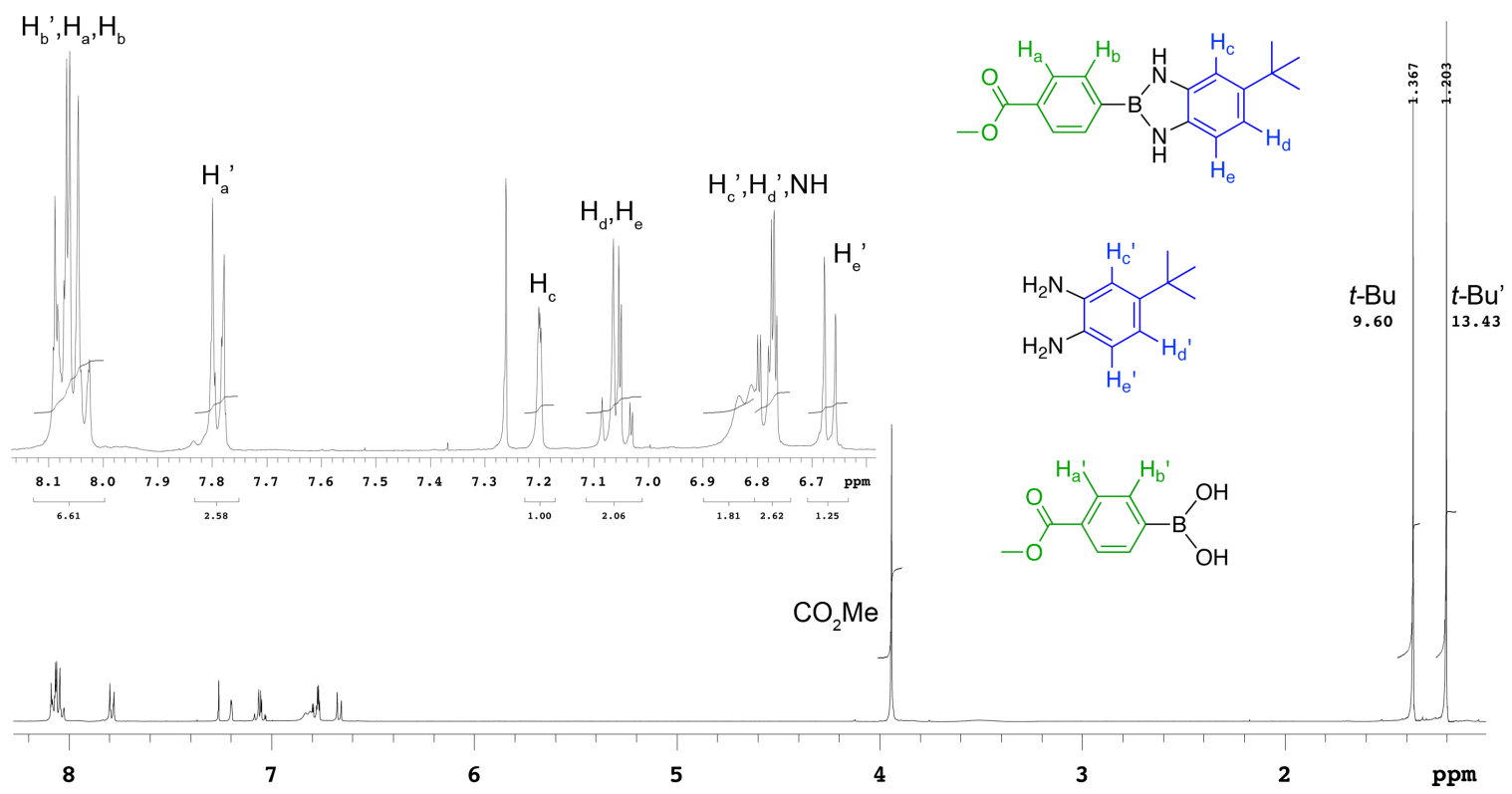

Figure S17. ${ }^{1} \mathrm{H}$ NMR spectrum of an equilibrated mixture of 4-methoxycarbonyl phenylboronic acid (5) and 4-tert-butyl-ortho-phenylenediamine (8) to give diazaborole product 12e. Percent diazaborole calculation based primarily on $t$-butyl peaks: $9.60 /(9.60+13.43)=41.7 \%$. Calculation based on $\mathrm{H}_{\mathrm{c}}$ of the diazaborole versus $\mathrm{H}_{\mathrm{e}}$, of diamine 8 gives $1.00 /(1.00+1.25)=44.4 \%$, though lower resolution and signal-to-noise makes this a less reliable assessment.

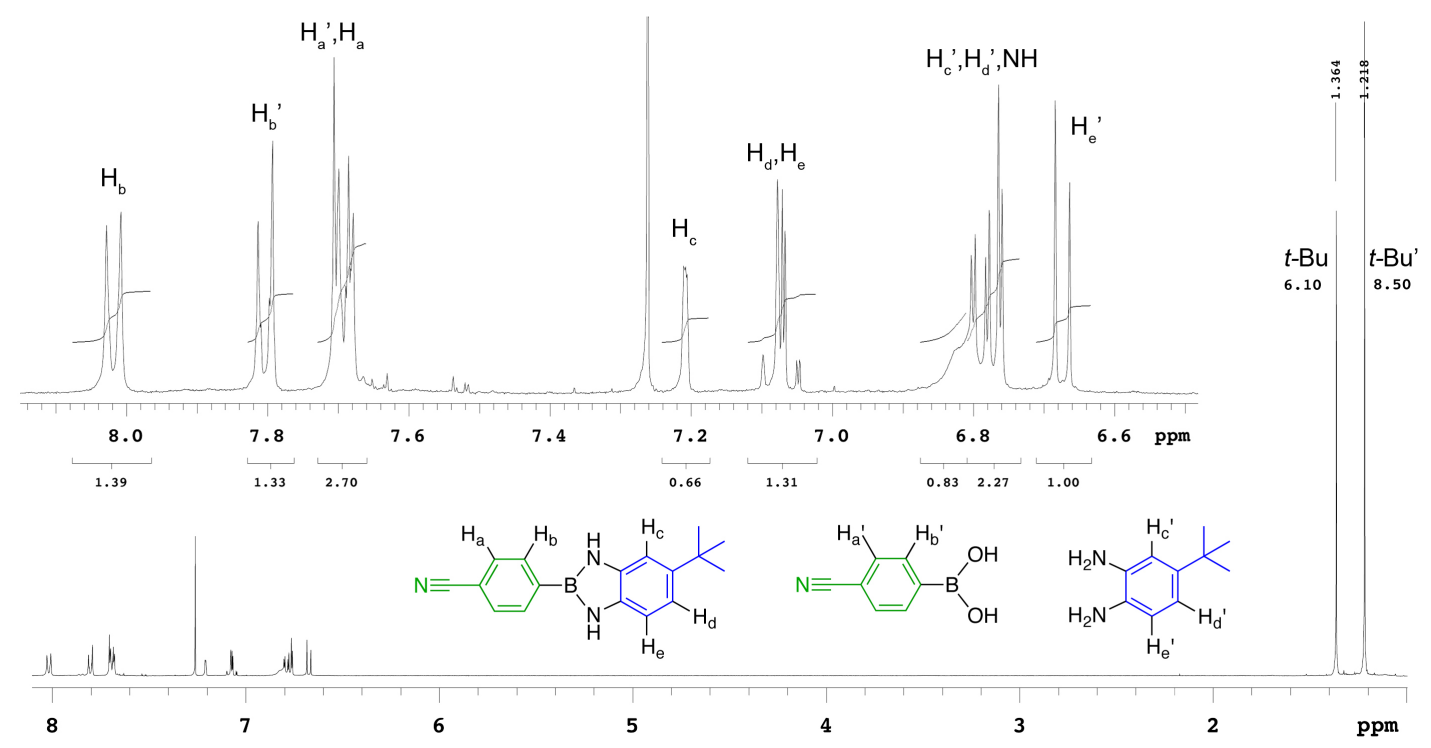

Figure S18. ${ }^{1} \mathrm{H}$ NMR spectrum of an equilibrated mixture of 4-cyanophenylboronic acid (6) and 4-tert-butyl-ortho-phenylenediamine (8) to give diazaborole product 12f. Percent diazaborole calculation based primarily on $t$-butyl peaks: $6.10 /(6.10+8.50)=41.7 \%$. Calculation based on $\mathrm{H}_{c}$ of the $\mathbf{1 2 f}$ versus $\mathrm{H}_{\mathrm{e}}$, of $\mathbf{8}$ gives: $0.66 /(0.66+1.00)=39.8 \%$, though lower resolution and signal-to-noise makes this a less reliable assessment. 
V. Concentration and $\boldsymbol{K}_{\text {eq. }}$. Serial dilution was used to investigate the influence of concentration on boronate ester formation and signal-to-noise in ${ }^{1} \mathrm{H}$ NMR spectra. A 0.10 $\mathrm{M}$ solution of 1:1 phenylboronic acid (3) and $t \mathrm{Bu}$-catechol (7) was diluted three times, each by a factor of two, to give equimolar solutions of four concentrations: $0.10 \mathrm{M}, 0.05$ $\mathrm{M}, 0.025 \mathrm{M}$, and $0.0125 \mathrm{M}$. The solutions were allowed to reach equilibrium overnight. Spectroscopic results showed the expected increase in percent boronate ester formation at lower concentrations (from $\sim 96 \%$ at $0.10 \mathrm{M}$ to $\sim 99 \%$ at $0.0125 \mathrm{M}$ ), however signal-tonoise becomes very poor at such dilution. While signal-to-noise improves at greater concentrations the solubility of free boronic acid species decreases rapidly. Experimental results obtained at $0.05 \mathrm{M}$ provide a reasonable balance of ${ }^{1} \mathrm{H}$ NMR resolution and compound solubility for determining the percentage of boronate ester formation.

Figures S19-S22 show equilibrated ${ }^{1} \mathrm{H}$ NMR spectra at concentrations ranging from 0.10 $\mathrm{M}$ to $0.0125 \mathrm{M}$, as well as the percentage of boronate ester formation and $K_{\text {eq }}$ at each concentration. As can be seen the equilibrium concentrations fall within a relatively narrow range of $69.5-75.5$, corresponding to a $\Delta G^{\circ}$ of $2.51-2.56 \mathrm{kcal} / \mathrm{mol}$.

Signal-to-noise was not a significant concern for diazaborole formation given that tertbutyl peaks of assembled and unassembled species are both well resolved and significantly above the ${ }^{1} \mathrm{H}$ NMR baseline noise.

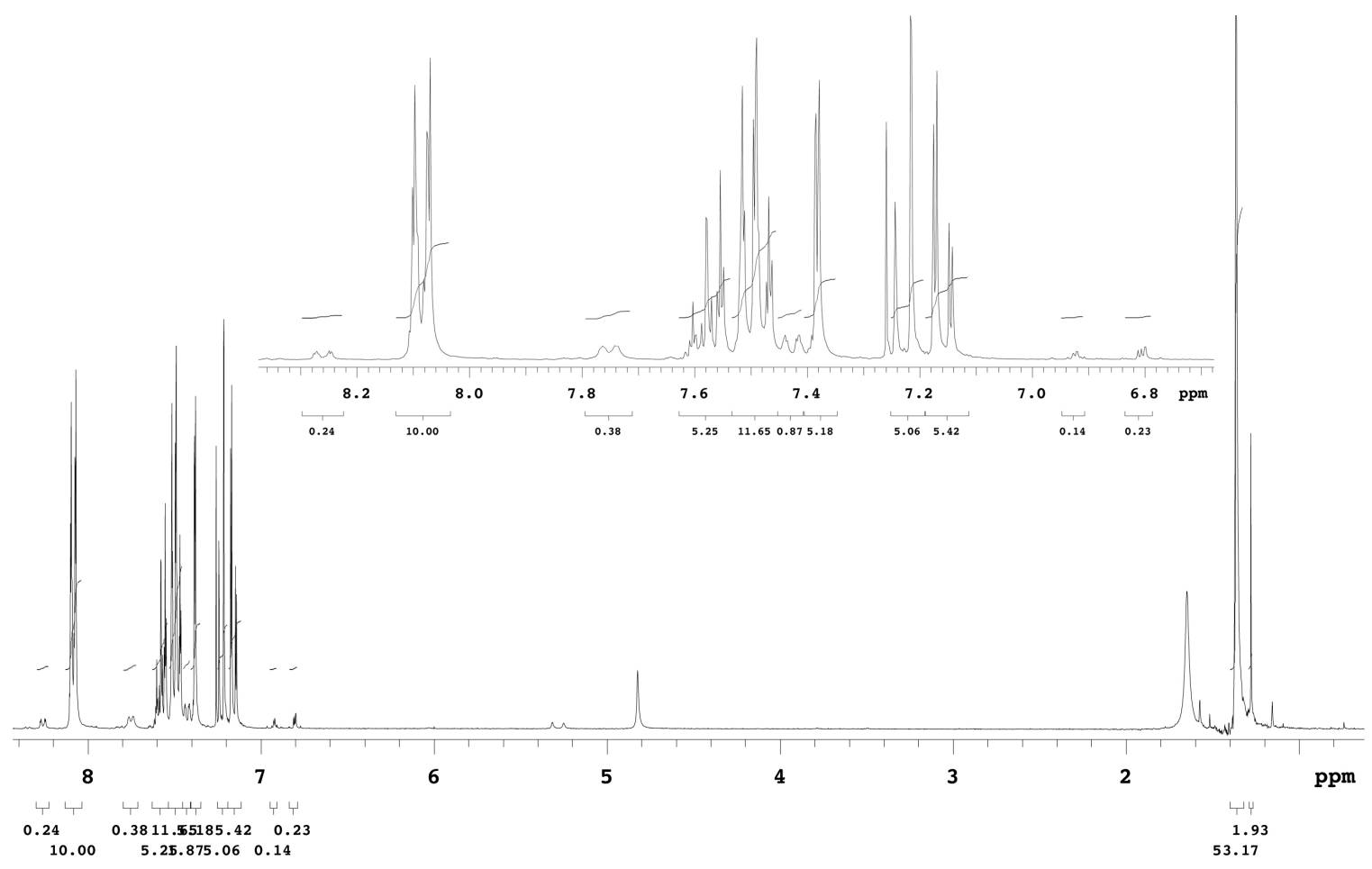

Figure S19. Solution concentration: $0.10 \mathrm{M}$. Percent boronate based on $t \mathrm{Bu}$ peaks: $53.17 /(53.17+1.93)=96.5 \%, K_{\text {eq }}=73.2$. Repeating the calculation based on $\mathrm{H}_{\mathrm{c}}$ of the 11c, boroxine, and boronic acid gives: $10.0 /(10.0+0.24 / 3+0.38)=95.6 \%$, though signal-to-noise for boroxine and boronic acid signals is poor. 


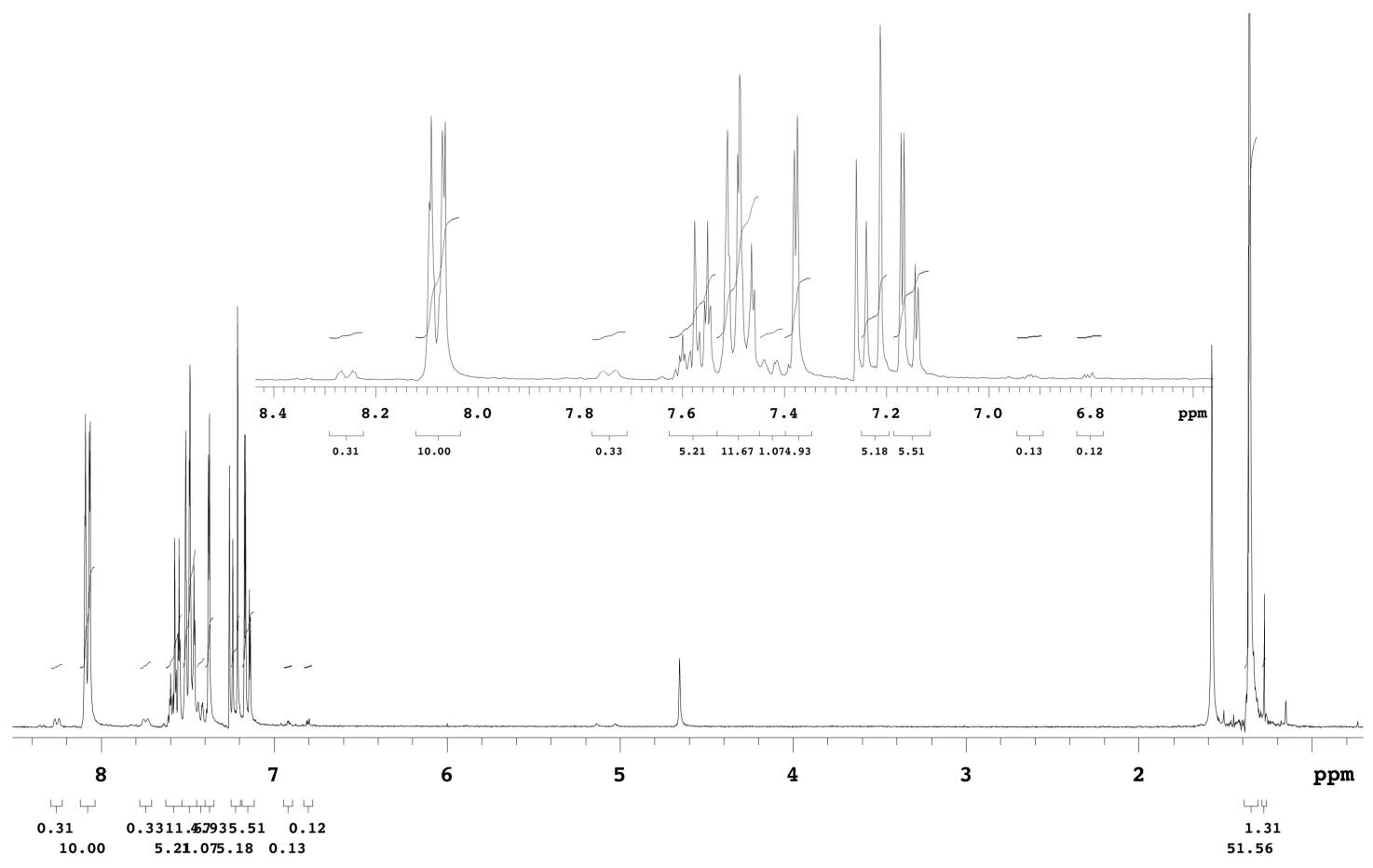

Figure S20. Solution concentration: $0.05 \mathrm{M}$. Percent boronate based on $t \mathrm{Bu}$ peaks: $51.56 /(51.56+1.31)=97.5 \%, K_{\text {eq }}=75.5$. Repeating the calculation based on $\mathrm{H}_{\mathrm{c}}$ of the 11c, boroxine, and boronic acid gives: $10.0 /(10.0+0.31 / 3+0.33)=95.8 \%$, though signal-to-noise for boroxine and boronic acid signals is poor.

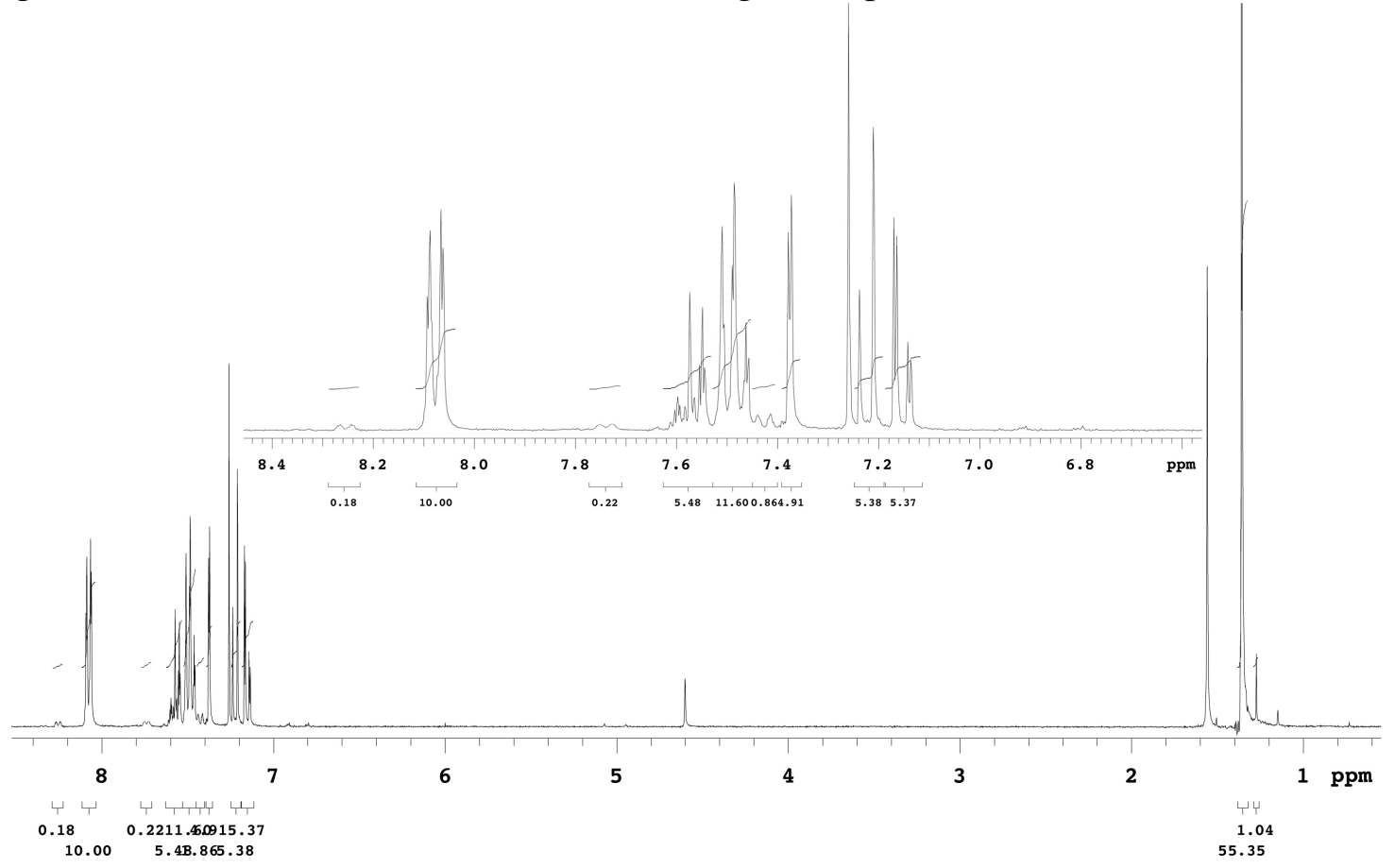

Figure S21. Solution concentration: $0.025 \mathrm{M}$. Percent boronate based on $t \mathrm{Bu}$ peaks: $55.35 /(55.35+1.04)=98.2 \%, K_{\mathrm{eq}}=69.5$. Repeating the calculation based on $\mathrm{H}_{\mathrm{c}}$ of the 11c, boroxine, and boronic acid: $10.0 /(10.0+0.18 / 3+0.22)=97.3 \%$, though signal-tonoise of aryl peaks is very poor and likely unreliable. 


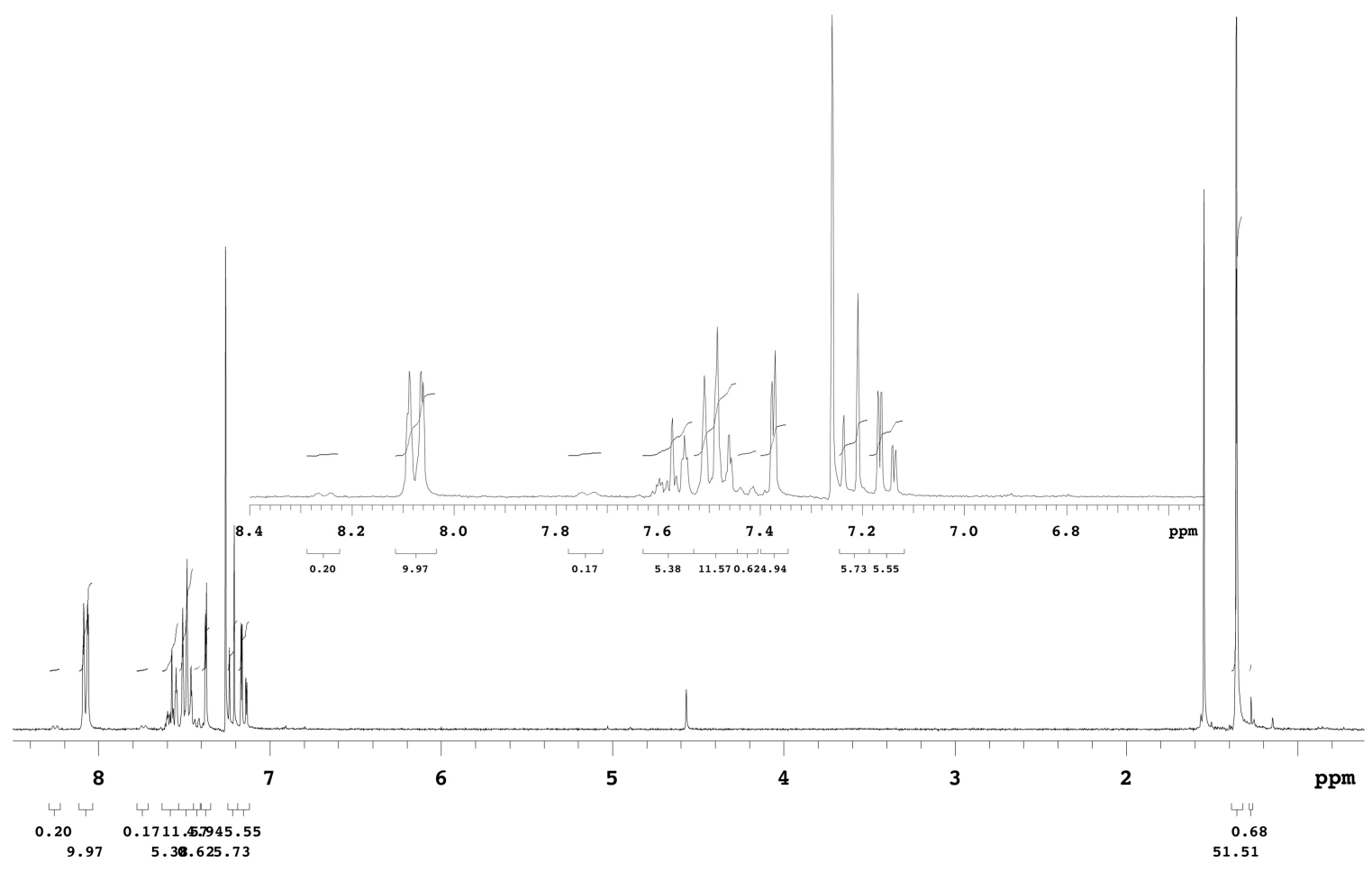

Figure S22. Solution concentration: $0.0125 \mathrm{M}$. Percent boronate based on $t \mathrm{Bu}$ peaks: $51.51 /(51.51+0.68)=98.7 \%, K_{\mathrm{eq}}=70.8$. Repeating the calculation based on $\mathrm{H}_{\mathrm{c}}$ of the 11c, boroxine, and boronic acid: $10.0 /(10.0+0.20 / 3+0.17)=97.7 \%$. The signal-to-noise of aryl peaks and $t \mathrm{Bu}$ peaks is so poor that we cannot recommend basing the equilibrium constant on ${ }^{1} \mathrm{H}$ NMR results obtained at this dilute concentration. 
VI. Table S1: Boronic Acid Conformations \& Relative Energetics. Relative free energies $\left(\Delta G^{\circ}\right)$, intramolecular hydrogen bonding, $\mathrm{C}-\mathrm{B}$, and $\mathrm{B}-\mathrm{O}$ distances $(\AA)$, and $\mathrm{O}-$ $\mathrm{B}-\mathrm{O}$ angles of endo-exo, syn, and anti conformations of acids 1-6 calculated at the B3LYP/6-311+G(d,p), M06-2X/6-31+G(d,p), CBS-QB3, and MP2/aug-cc-pvdz levels.

\section{B3LYP/6-311+G(d,p)}

\begin{tabular}{cccccccc}
\hline & & $\mathbf{1}$ & $\mathbf{2}$ & $\mathbf{3}$ & $\mathbf{4}$ & $\mathbf{5}$ & $\mathbf{6}$ \\
& & $(\mathrm{R}=\mathrm{OMe})$ & $(\mathrm{R}=t-\mathrm{Bu})$ & $(\mathrm{R}=\mathrm{H})$ & $(\mathrm{R}=\mathrm{F})$ & $\left(\mathrm{R}=\mathrm{CO}_{2} \mathrm{Me}\right)$ & $(\mathrm{R}=\mathrm{CN})$ \\
\hline \multirow{6}{*}{ endo- } & $\mathrm{O}-\mathrm{H} \bullet \bullet-O$ & 2.383 & 2.385 & 2.388 & 2.390 & 2.393 & 2.401 \\
exo & $\mathrm{C}-\mathrm{B}$ & 1.561 & 1.564 & 1.567 & 1.567 & 1.572 & 1.574 \\
& $\mathrm{~B}-\mathrm{O}_{\mathrm{A}}$ & 1.376 & 1.375 & 1.374 & 1.373 & 1.371 & 1.370 \\
& $\mathrm{~B}^{-} \mathrm{O}_{\mathrm{B}}$ & 1.372 & 1.371 & 1.370 & 1.370 & 1.368 & 1.366 \\
& $\angle \mathrm{OBO}$ & 116.7 & 116.9 & 117.0 & 117.1 & 117.3 & 117.7 \\
\hline \multirow{6}{*}{ syn } & $\Delta \mathrm{G}^{\circ}$ & 1.1 & 1.7 & 1.4 & 1.1 & 1.3 & 0.9 \\
& $\mathrm{C}-\mathrm{B}$ & 1.558 & 1.561 & 1.564 & 1.563 & 1.569 & 1.570 \\
& $\mathrm{~B}-\mathrm{O}$ & 1.376 & 1.375 & 1.374 & 1.373 & 1.371 & 1.369 \\
& $\angle \mathrm{OBO}$ & 123.2 & 123.2 & 123.4 & 123.7 & 124.0 & 124.4 \\
\hline \multirow{6}{*}{ anti } & $\Delta \mathrm{G}^{\circ}$ & 2.1 & 2.2 & 3.1 & 2.5 & 2.2 & 2.7 \\
& $\mathrm{C}-\mathrm{B}$ & 1.567 & 1.570 & 1.576 & 1.572 & 1.577 & 1.579 \\
& $\mathrm{~B}-\mathrm{O}$ & 1.373 & 1.372 & 1.371 & 1.370 & 1.368 & 1.367 \\
& $\angle \mathrm{OBO}$ & 113.7 & 114.0 & 113.4 & 114.2 & 114.5 & 114.7 \\
& dihedral & 23.5 & 26.1 & 29.9 & 29.5 & 31.8 & 32.6 \\
\hline
\end{tabular}

M06-2X/6-31+G(d,p)

\begin{tabular}{|c|c|c|c|c|c|c|c|}
\hline & & $\begin{array}{c}1 \\
(\mathrm{R}=\mathrm{OMe})\end{array}$ & $\begin{array}{c}2 \\
(R=t-B u)\end{array}$ & $\begin{array}{c}3 \\
(R=H)\end{array}$ & $\begin{array}{c}4 \\
(R=F)\end{array}$ & $\begin{array}{c}\mathbf{5} \\
\left(\mathrm{R}=\mathrm{CO}_{2} \mathrm{Me}\right)\end{array}$ & $\begin{array}{c}6 \\
(\mathrm{R}=\mathrm{CN})\end{array}$ \\
\hline \multirow{5}{*}{$\begin{array}{l}\text { endo- } \\
\text { exo }\end{array}$} & $\mathrm{O}-\mathrm{H} \bullet \bullet \bullet \mathrm{O}$ & 2.392 & 2.393 & 2.393 & 2.396 & 2.401 & 2.407 \\
\hline & $C-B$ & 1.564 & 1.568 & 1.570 & 1.570 & 1.575 & 1.577 \\
\hline & $B-O_{A}$ & 1.372 & 1.371 & 1.370 & 1.369 & 1.368 & 1.366 \\
\hline & $\mathrm{B}-\mathrm{O}_{\mathrm{B}}$ & 1.368 & 1.367 & 1.367 & 1.366 & 1.365 & 1.364 \\
\hline & $\angle O B O$ & 117.4 & 117.5 & 117.6 & 117.7 & 118 & 118.3 \\
\hline \multirow{4}{*}{ syn } & $\Delta \mathrm{G}^{\circ}$ & 1.0 & 1.0 & 1.3 & 0.9 & 0.9 & 0.7 \\
\hline & $C-B$ & 1.561 & 1.564 & 1.567 & 1.566 & 1.571 & 1.573 \\
\hline & $\mathrm{B}-\mathrm{O}$ & 1.371 & 1.371 & 1.370 & 1.369 & 1.367 & 1.366 \\
\hline & $\angle O B O$ & 123.9 & 123.9 & 124.1 & 124.3 & 124.7 & 125.0 \\
\hline \multirow{5}{*}{ anti } & $\Delta \mathrm{G}^{\circ}$ & 2.4 & 2.5 & 2.6 & 2.8 & 2.7 & 3.0 \\
\hline & $C-B$ & 1.571 & 1.574 & 1.576 & 1.576 & 1.580 & 1.582 \\
\hline & $\mathrm{B}-\mathrm{O}$ & 1.368 & 1.367 & 1.367 & 1.366 & 1.365 & 1.364 \\
\hline & $\angle O B O$ & 114.6 & 114.9 & 115.0 & 115.0 & 115.3 & 115.5 \\
\hline & dihedral & 25.6 & 28.30 & 29.7 & 30.1 & 31.0 & 32.3 \\
\hline
\end{tabular}




\begin{tabular}{cccccccc}
\hline & & $\mathbf{1}$ & $\mathbf{2}$ & $\mathbf{3}$ & $\mathbf{4}$ & $\mathbf{5}$ & $\mathbf{6}$ \\
& & $(\mathrm{R}=\mathrm{OMe})$ & $(\mathrm{R}=t-\mathrm{Bu})$ & $(\mathrm{R}=\mathrm{H})$ & $(\mathrm{R}=\mathrm{F})$ & $\left(\mathrm{R}=\mathrm{CO}_{2} \mathrm{Me}\right)$ & $(\mathrm{R}=\mathrm{CN})$ \\
\hline \multirow{6}{*}{ endo- } & $\mathrm{O}-\mathrm{H} \bullet \bullet \bullet O$ & 2.376 & 2.378 & 2.380 & 2.382 & 2.386 & 2.397 \\
exo & $\mathrm{C}-\mathrm{B}$ & 1.562 & 1.566 & 1.569 & 1.568 & 1.573 & 1.575 \\
& $\mathrm{~B}-\mathrm{O}_{\mathrm{A}}$ & 1.373 & 1.372 & 1.371 & 1.371 & 1.369 & 1.367 \\
& $\mathrm{~B}-\mathrm{O}_{\mathrm{B}}$ & 1.369 & 1.368 & 1.368 & 1.367 & 1.366 & 1.364 \\
& $\angle \mathrm{OBO}$ & 117.0 & 117.2 & 117.3 & 117.4 & 117.6 & 118.1 \\
\hline \multirow{6}{*}{ syn } & $\Delta \mathrm{G}^{\circ}$ & 1.4 & 1.6 & 1.4 & 1.0 & 1.5 & 0.8 \\
& $\mathrm{C}-\mathrm{B}$ & 1.559 & 1.563 & 1.565 & 1.564 & 1.570 & 1.572 \\
& $\mathrm{~B}-\mathrm{O}$ & 1.373 & 1.372 & 1.371 & 1.370 & 1.368 & 1.367 \\
& $\angle \mathrm{OBO}$ & 123.5 & 123.6 & 123.7 & 123.9 & 124.4 & 124.7 \\
\hline \multirow{6}{*}{ anti } & $\Delta \mathrm{G}^{\circ}$ & 2.1 & 2.0 & 2.0 & 2.1 & 2.2 & 2.4 \\
& $\mathrm{C}-\mathrm{B}$ & 1.568 & 1.571 & 1.573 & 1.573 & 1.577 & 1.579 \\
& $\mathrm{~B}-\mathrm{O}$ & 1.370 & 1.369 & 1.368 & 1.368 & 1.366 & 1.365 \\
& $\angle \mathrm{OBO}$ & 114.3 & 114.6 & 114.7 & 114.7 & 115.0 & 115.2 \\
& dihedral & 26.9 & 29.1 & 30.3 & 30.4 & 32.5 & 32.9 \\
\hline
\end{tabular}

MP2/aug-cc-pvdz

\begin{tabular}{|c|c|c|c|c|c|c|c|}
\hline & & $\begin{array}{c}1 \\
(\mathrm{R}=\mathrm{OMe})\end{array}$ & $\begin{array}{c}2 \\
(\mathrm{R}=t-\mathrm{Bu})\end{array}$ & $\begin{array}{c}3 \\
(R=H)\end{array}$ & $\begin{array}{c}4 \\
(R=F)\end{array}$ & $\begin{array}{c}\mathbf{5} \\
\left(\mathrm{R}=\mathrm{CO}_{2} \mathrm{Me}\right)\end{array}$ & $\begin{array}{c}6 \\
(\mathrm{R}=\mathrm{CN})\end{array}$ \\
\hline \multirow{5}{*}{$\begin{array}{l}\text { endo- } \\
\text { exo }\end{array}$} & $\mathrm{O}-\mathrm{H} \bullet \bullet \mathrm{O}$ & 2.391 & 2.395 & 2.396 & 2.399 & 2.400 & 2.407 \\
\hline & C-B & 1.570 & 1.572 & 1.575 & 1.574 & 1.577 & 1.578 \\
\hline & $\mathrm{B}-\mathrm{O}_{\mathrm{A}}$ & 1.389 & 1.388 & 1.387 & 1.386 & 1.385 & 1.384 \\
\hline & $\mathrm{B}-\mathrm{O}_{\mathrm{B}}$ & 1.385 & 1.384 & 1.383 & 1.383 & 1.382 & 1.381 \\
\hline & $\angle O B O$ & 117.3 & 117.5 & 117.6 & 117.7 & 117.8 & 118.1 \\
\hline \multirow{4}{*}{ syn } & $\Delta \mathrm{G}^{\circ}$ & 1.1 & 1.2 & 1.2 & 0.9 & 1.0 & 0.7 \\
\hline & $C-B$ & 1.567 & 1.570 & 1.572 & 1.571 & 1.573 & 1.575 \\
\hline & $\mathrm{B}-\mathrm{O}$ & 1.388 & 1.388 & 1.387 & 1.386 & 1.385 & 1.384 \\
\hline & $\angle O B O$ & 124.4 & 124.4 & 124.5 & 124.8 & 125.0 & 125.4 \\
\hline \multirow{5}{*}{ anti } & $\Delta \mathrm{G}^{\circ}$ & 1.5 & 2.6 & 1.6 & 1.8 & 1.8 & 2.1 \\
\hline & C-B & 1.574 & 1.579 & 1.578 & 1.578 & 1.580 & 1.582 \\
\hline & $\mathrm{B}-\mathrm{O}$ & 1.385 & 1.385 & 1.384 & 1.383 & 1.383 & 1.381 \\
\hline & $\angle O B O$ & 113.6 & 113.1 & 113.9 & 114.0 & 114.1 & 114.3 \\
\hline & dihedral & 25.9 & 27.2 & 29.9 & 30.4 & 31.3 & 32.1 \\
\hline
\end{tabular}




\section{Bond Lengths of 12a-f, 13a-f, and 14a-f.}

Table S2. Bond lengths ( $\AA$ ) of B-N and C-B bonds in diazaboroles 12a-f demonstrating the progressive shortening of $\mathrm{B}-\mathrm{N}$ bond distances and lengthening of $\mathrm{C}-\mathrm{B}$ bond distances with increasing electron withdrawing nature of the para-substituent of the boronic acid.

\begin{tabular}{ccccccc} 
& $\mathbf{1 2 a}$ & $\mathbf{1 2 b}$ & $\mathbf{1 2 c}$ & $\mathbf{1 2 d}$ & $\mathbf{1 2 e}$ & $\mathbf{1 2 f}$ \\
\hline $\mathrm{R}$ & $\mathrm{OMe}$ & $t$-Bu & $\mathrm{H}$ & $\mathrm{F}$ & $\mathrm{CO}_{2} \mathrm{Me}$ & $\mathrm{CN}$ \\
\hline $\mathrm{B}-\mathrm{N}(\AA)$ & 1.442 & 1.440 & 1.439 & 1.438 & 1.437 & 1.436 \\
$\mathrm{C}-\mathrm{B}(\AA)$ & 1.552 & 1.554 & 1.556 & 1.555 & 1.558 & 1.559 \\
\hline
\end{tabular}

Table S3. Bond lengths ( $\AA$ ) of B-O, B-S, and C-B bonds in oxathiaboroles 13a-f demonstrating the progressive shortening of $\mathrm{B}-\mathrm{O}$ and $\mathrm{B}-\mathrm{S}$ bond distances and lengthening of $\mathrm{C}-\mathrm{B}$ bond distances with increasing electron withdrawing nature of the para-substituent of the boronic acid.

\begin{tabular}{ccccccc} 
& $\mathbf{1 3 a}$ & $\mathbf{1 3 b}$ & $\mathbf{1 3 c}$ & $\mathbf{1 3 d}$ & $\mathbf{1 3 e}$ & $\mathbf{1 3 f}$ \\
\hline $\mathrm{R}$ & $\mathrm{OMe}$ & $t-\mathrm{Bu}$ & $\mathrm{H}$ & $\mathrm{F}$ & $\mathrm{CO}_{2} \mathrm{Me}$ & $\mathrm{CN}$ \\
\hline $\mathrm{B}-\mathrm{O}(\AA)$ & 1.385 & 1.383 & 1.382 & 1.382 & 1.381 & 1.379 \\
$\mathrm{~B}-\mathrm{S}(\AA)$ & 1.840 & 1.837 & 1.836 & 1.836 & 1.833 & 1.831 \\
$\mathrm{C}-\mathrm{B}(\AA)$ & 1.537 & 1.541 & 1.544 & 1.544 & 1.548 & 1.550 \\
\hline
\end{tabular}

Table S4. Bond lengths ( $\AA$ ) of B-S and C-B bonds in thiaboroles 14a-f demonstrating the progressive shortening of $\mathrm{B}-\mathrm{S}$ bond distances and lengthening of $\mathrm{C}-\mathrm{B}$ bond distances with increasing electron withdrawing nature of the para-substituent of the boronic acid.

\begin{tabular}{ccccccc} 
& $\mathbf{1 4 a}$ & $\mathbf{1 4 b}$ & $\mathbf{1 4 c}$ & $\mathbf{1 4 d}$ & $\mathbf{1 4 e}$ & $\mathbf{1 4 f}$ \\
\hline $\mathrm{R}$ & $\mathrm{OMe}$ & $t-\mathrm{Bu}$ & $\mathrm{H}$ & $\mathrm{F}$ & $\mathrm{CO}_{2} \mathrm{Me}$ & $\mathrm{CN}$ \\
\hline $\mathrm{B}-\mathrm{S}(\AA)$ & 1.819 & 1.817 & 1.815 & 1.815 & 1.812 & 1.810 \\
$\mathrm{C}-\mathrm{B}(\AA)$ & 1.542 & 1.547 & 1.550 & 1.549 & 1.554 & 1.556 \\
\hline
\end{tabular}




\section{Calculated Electron Density Maps.}

Electron density maps of diazaboroles 12a-f and dithiaboroles 14a-f were calculated at the B3LYP/6-311+G(d,p) level. Isovalues for all structures were set to -0.02 to 0.02 .

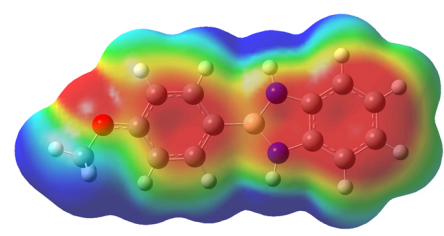

$12 a$

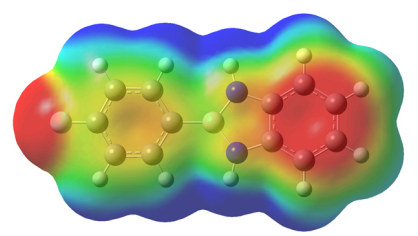

$12 d$

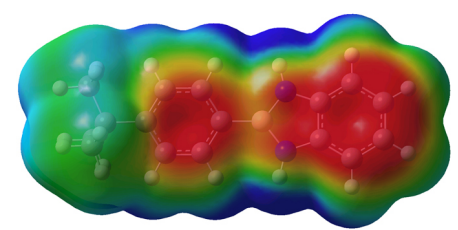

$12 b$

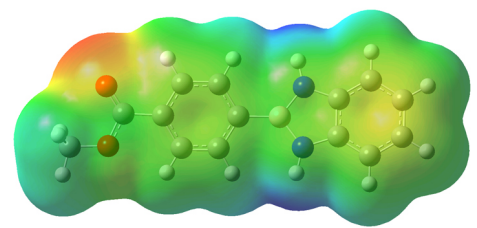

$12 e$

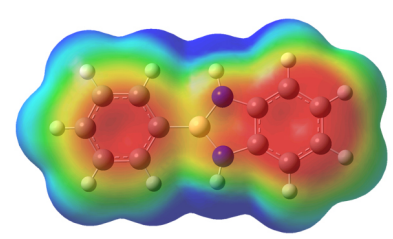

$12 c$

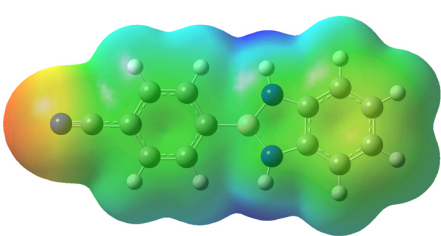

$12 f$

Figure S23. Electron density maps of diazaboroles 12a-f revealing the progressive decrease in electron density surrounding the diazaborole $\mathrm{B}(\mathrm{NH})_{2}$ functionality and the ortho-phenylene diamine ring with greater withdrawing strength of the substituent at the para-position of each boronic acid.

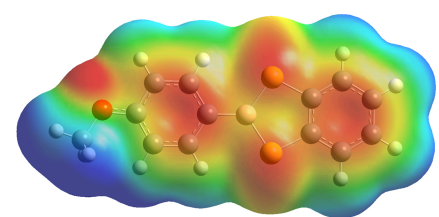

$14 a$

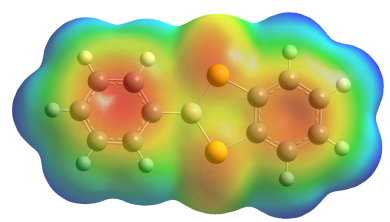

$14 d$

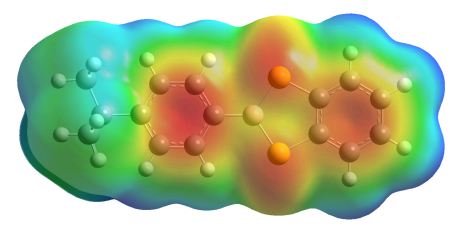

$14 b$

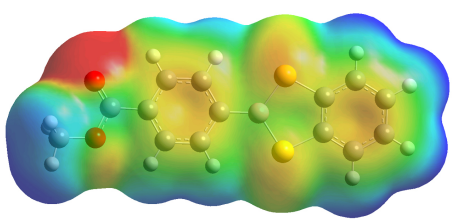

$14 e$

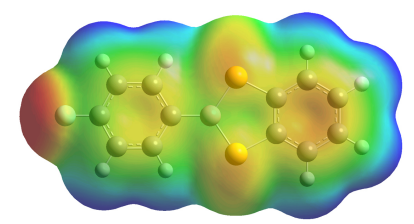

$14 c$

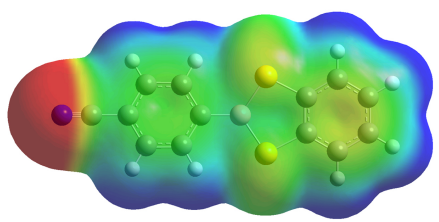

$14 f$

Figure S24. Electron density maps of dithiaboroles 14a-f also revealing the influence of the boronic acid para-substituent. The electron density surrounding the boron atom of the dithiaborole moiety is less than is calculated for diazaboroles 12a-f yet greater than is calculated for boronate esters 11a-f (see Figure 4 of the main text). 
IX. Stationary Point Summaries: Stationary points were optimized and energies were calculated as described in the text. Geometries below are for stationary points optimized at the M06-2X/6-31+G(d,p) level are given below in Cartesian coordinates. Structures optimized at the other three levels of theory are not substantially different from the following M06-2X geometries, therefore only one set of Cartesians is provided, as four sets for each stationary point would be relatively redundant. Free energies at each level of theory are given in Hartrees.

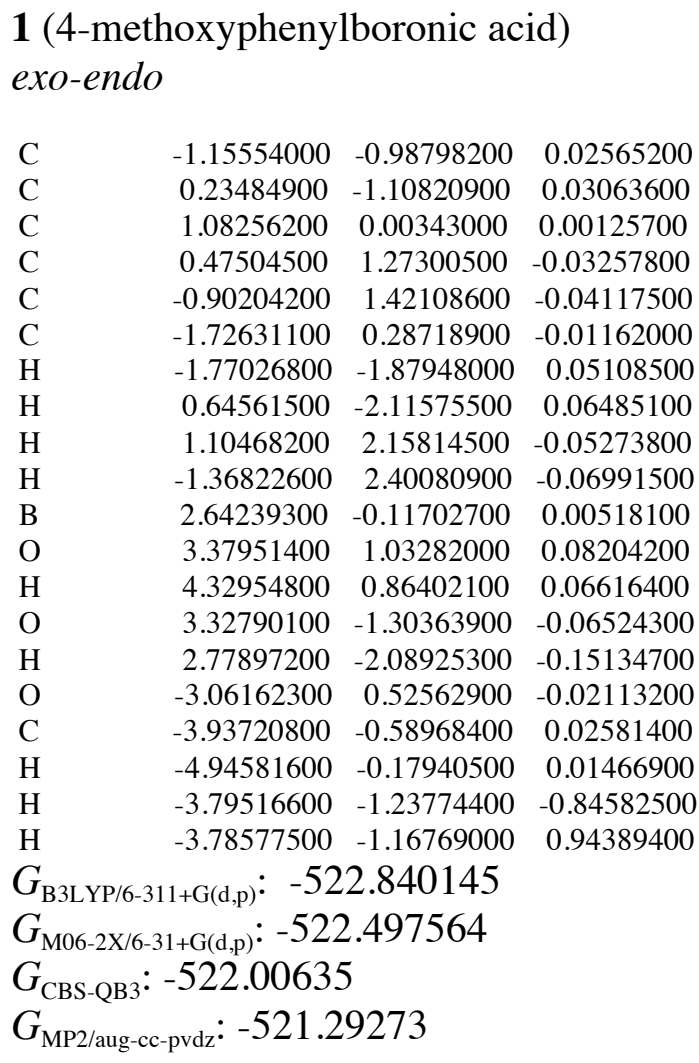

$\begin{array}{lrrr}\text { syn } & & & \\ & & & \\ \mathrm{C} & -1.14784700 & -0.98651000 & -0.00038600 \\ \mathrm{C} & 0.24336300 & -1.09793800 & -0.00029900 \\ \mathrm{C} & 1.07819500 & 0.02408100 & -0.00010300 \\ \mathrm{C} & 0.46510100 & 1.28982900 & -0.00004700 \\ \mathrm{C} & -0.91392500 & 1.42712100 & -0.00013200 \\ \mathrm{C} & -1.72778900 & 0.28553700 & -0.00027400 \\ \mathrm{H} & -1.75783900 & -1.88185600 & -0.00056400 \\ \mathrm{H} & 0.68918400 & -2.08935200 & -0.00037900 \\ \mathrm{H} & 1.08778800 & 2.18045200 & 0.00010800 \\ \mathrm{H} & -1.38914200 & 2.40299800 & -0.00005100 \\ \mathrm{~B} & 2.63207600 & -0.12261900 & 0.00010200 \\ \mathrm{O} & 3.38653400 & 1.02266300 & 0.00026800 \\ \mathrm{H} & 4.34253300 & 0.90502500 & 0.00050400 \\ \mathrm{O} & 3.16291800 & -1.38779300 & 0.00005600 \\ \mathrm{H} & 4.12431700 & -1.44848300 & 0.00034500\end{array}$

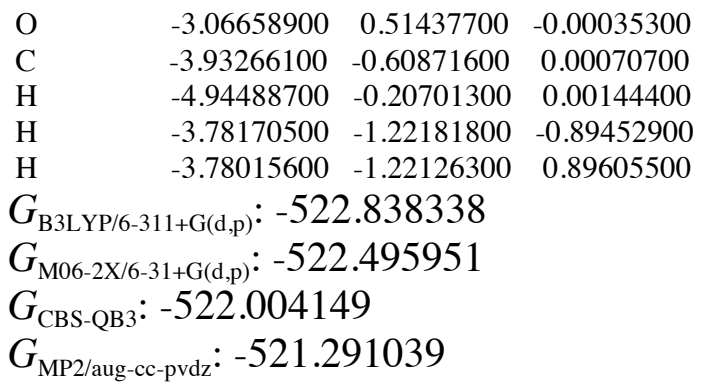

2 (4-tert-butylphenylboronic acid)
exo-endo
$\begin{array}{lrrr}\mathrm{C} & & & \\ \mathrm{C} & -0.37407000 & 1.23155100 & -0.00038800 \\ \mathrm{H} & 1.02161000 & 1.22334300 & -0.00040800\end{array}$ 


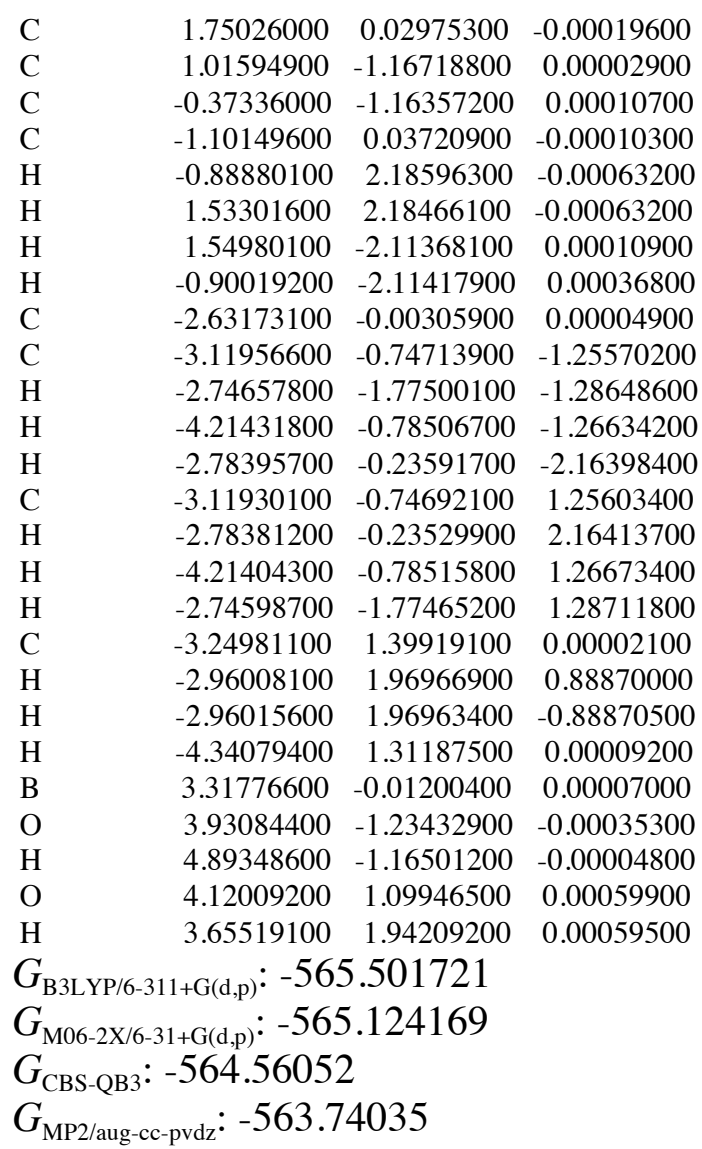

syn

$\begin{array}{lrrr}\text { C } & -0.36863100 & 1.22924400 & -0.00453800 \\ \mathrm{C} & 1.02732500 & 1.21645300 & -0.00387500 \\ \mathrm{C} & 1.74544100 & 0.01649300 & 0.00020100 \\ \mathrm{C} & 1.00885900 & -1.17795400 & 0.00423900 \\ \mathrm{C} & -0.38077600 & -1.16748700 & 0.00450400 \\ \mathrm{C} & -1.10191300 & 0.03745100 & -0.00027300 \\ \mathrm{H} & -0.88065100 & 2.18534300 & -0.00836700 \\ \mathrm{H} & 1.56777600 & 2.15981100 & -0.00711700 \\ \mathrm{H} & 1.53795600 & -2.12743900 & 0.00756000 \\ \mathrm{H} & -0.91294900 & -2.11519600 & 0.00822200 \\ \mathrm{C} & -2.63272300 & 0.00204800 & -0.00014800 \\ \mathrm{C} & -3.12386900 & -0.75099000 & -1.24931500 \\ \mathrm{H} & -2.75559100 & -1.78081300 & -1.27058600 \\ \mathrm{H} & -4.21885900 & -0.78403600 & -1.26048000 \\ \mathrm{H} & -2.78517200 & -0.24968500 & -2.16196000 \\ \mathrm{C} & -3.12368900 & -0.72930500 & 1.26187800 \\ \mathrm{H} & -2.78661000 & -0.21099900 & 2.16559500 \\ \mathrm{H} & -4.21864500 & -0.76402200 & 1.27279700 \\ \mathrm{H} & -2.75346500 & -1.75787400 & 1.30192600 \\ \mathrm{C} & -3.24697700 & 1.40597800 & -0.01223800 \\ \mathrm{H} & -2.95673200 & 1.98261900 & 0.87220300 \\ \mathrm{H} & -2.95367200 & 1.96851900 & -0.90474200 \\ \mathrm{H} & -4.33830700 & 1.32178900 & -0.01335600 \\ \mathrm{~B} & 3.30936700 & -0.00273900 & 0.00012500\end{array}$

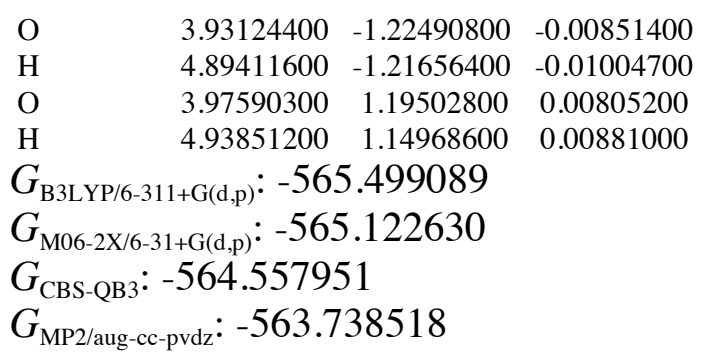

anti

$\begin{array}{llll}\text { C } & -0.35625600 & 1.20668100 & -0.20971400\end{array}$

$\begin{array}{llll}\text { C } & 1.03928900 & 1.19048200 & -0.20110300\end{array}$

$\begin{array}{llll}\text { C } & 1.76063400 & 0.00872900 & 0.00246900\end{array}$

$\begin{array}{lllll}\text { C } & 1.01615900 & -1.16423700 & 0.20569400\end{array}$

C $\quad-0.37361100 \quad-1.15014400 \quad 0.20918900$

$\begin{array}{lllll}\mathrm{C} & -1.09294600 & 0.03627200 & -0.00254200\end{array}$

$\begin{array}{llll}\mathrm{H} & -0.86286000 & 2.14954900 & -0.38353500\end{array}$

$\mathrm{H} \quad \begin{array}{llll}\mathrm{H} & 1.56508900 & 2.12688900 & -0.37940500\end{array}$

$\begin{array}{llll}\mathrm{H} & 1.52497500 & -2.10972900 & 0.38495400\end{array}$

$\begin{array}{llll}\mathrm{H} & -0.90689600 & -2.08122400 & 0.38119700\end{array}$

$\begin{array}{lllll}\mathrm{C} & -2.62299700 & 0.00376100 & -0.00134100\end{array}$

$\begin{array}{lllll}\mathrm{C} & & -3.10898100 & -0.94591900 & -1.11084400\end{array}$

$\mathrm{H} \quad-2.74118300 \quad-1.96493200 \quad-0.95856700$

$\mathrm{H} \quad-4.20380800 \quad-0.97977100 \quad-1.12095700$

$\mathrm{H} \quad-2.76538600 \quad-0.60268000 \quad-2.09209500$

$\begin{array}{llll}\mathrm{C} & -3.11903600 & -0.50927200 & 1.36249900\end{array}$

$\begin{array}{llll}\mathrm{H} & -2.78119900 & 0.14767200 & 2.17067300\end{array}$

$\begin{array}{llll}\mathrm{H} & -4.21399700 & -0.53622700 & 1.37505200\end{array}$

$\begin{array}{llll}\mathrm{H} & -2.75460400 & -1.51968200 & 1.57019700\end{array}$

$\begin{array}{lllll}\mathrm{C} & -3.23257700 & 1.38784000 & -0.24878600\end{array}$

$\begin{array}{llll}\mathrm{H} & -2.94266900 & 2.10277800 & 0.52839400\end{array}$

$\mathrm{H} \quad-2.93490700 \quad 1.79257000 \quad-1.22175900$

$\begin{array}{lllll}\mathrm{H} & -4.32412800 & 1.30891000 & -0.23945200\end{array}$

B $\quad 3.33439700 \quad-0.00495200 \quad-0.00039600$

O $\quad 4.05987900 \quad-1.10434400 \quad-0.36783600$

$\mathrm{H} \quad 3.52504200 \quad-1.82936600 \quad-0.70633900$

$\begin{array}{lllll}\mathrm{O} & 4.08092000 & 1.08170300 & 0.36252000\end{array}$

$\begin{array}{llll}\mathrm{H} & 3.56009400 & 1.81596900 & 0.70301200\end{array}$

$G_{\mathrm{B} 3 \mathrm{LYP} / 6-311+\mathrm{G}(\mathrm{d}, \mathrm{p})}:-565.498207$

$G_{\mathrm{M} 06-2 \mathrm{X} / 6-31+\mathrm{G}(\mathrm{d}, \mathrm{p})}:-565.120261$

$G_{\mathrm{CBS}-\mathrm{QB} 3}:-564.557320$

$G_{\mathrm{MP2} / \text { aug-cc-pvdz }}:-563.736221$

\section{3 (phenylboronic acid)} exo-endo

$\begin{array}{lrrr}\mathrm{C} & -1.92745100 & 1.18725900 & 0.21670900 \\ \mathrm{C} & -0.53366000 & 1.18320200 & 0.21007400 \\ \mathrm{C} & 0.19085200 & 0.00000300 & 0.00000200 \\ \mathrm{C} & -0.53365500 & -1.18319800 & -0.21007700 \\ \mathrm{C} & -1.92744600 & -1.18726200 & -0.21671000 \\ \mathrm{C} & -2.62646200 & -0.00000300 & 0.00000100 \\ \mathrm{H} & -2.46697500 & 2.11289200 & 0.39203700\end{array}$




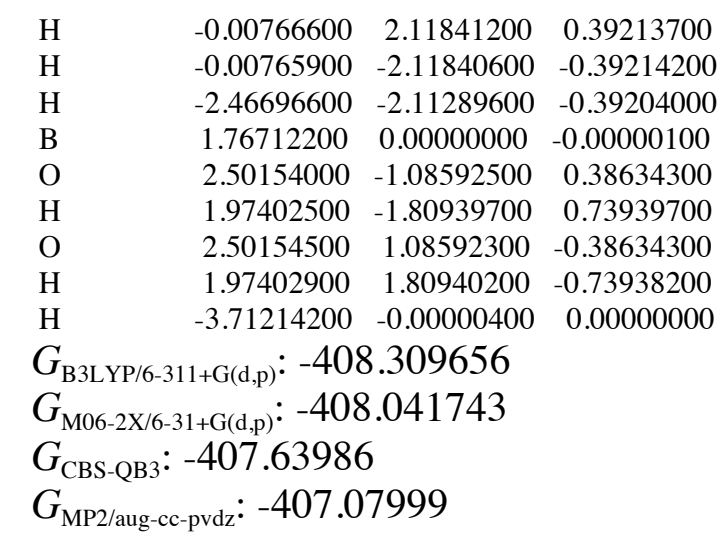

$\begin{array}{lrrr}\text { syn } & & & \\ \mathrm{C} & -1.94278200 & 1.20792300 & 0.03155500 \\ \mathrm{C} & -0.54886700 & 1.20383800 & 0.02837100 \\ \mathrm{C} & 0.16987000 & -0.00000300 & -0.00002200 \\ \mathrm{C} & -0.54887200 & -1.20384100 & -0.02839400 \\ \mathrm{C} & -1.94278700 & -1.20792000 & -0.03154300 \\ \mathrm{C} & -2.64073600 & 0.00000300 & 0.00001600 \\ \mathrm{H} & -2.48536200 & 2.14821700 & 0.05809900 \\ \mathrm{H} & -0.00511200 & 2.14463200 & 0.05017800 \\ \mathrm{H} & -0.00512200 & -2.14463700 & -0.05021900 \\ \mathrm{H} & -2.48537200 & -2.14821200 & -0.05807500 \\ \mathrm{~B} & 1.73662600 & -0.00000400 & -0.00005800 \\ \mathrm{O} & 2.37911300 & -1.20845700 & 0.05963200 \\ \mathrm{H} & 3.34194100 & -1.18023100 & 0.07610900 \\ \mathrm{O} & 2.37911100 & 1.20845700 & -0.05959800 \\ \mathrm{H} & 3.34194100 & 1.18024000 & -0.07600400 \\ \mathrm{H} & -3.72679700 & 0.00000500 & 0.00003000 \\ G_{\text {B3LYP/6-311+G(d,p) }}:-408.307472 & \\ G_{\text {M06-2X/6-31+G(d,p) }}:-408.039635 & \\ G_{\mathrm{CBS}-\mathrm{QB} 3}:-407.637595 & \\ G_{\mathrm{MP} 2 / \mathrm{aug}-\mathrm{cc}-\mathrm{pvdz}}:-407.078061 & \end{array}$

\section{anti}

$\begin{array}{lrrr}\mathrm{C} & 1.95970100 & 1.19460100 & -0.00004300 \\ \mathrm{C} & 0.56592300 & 1.20931000 & 0.00000600 \\ \mathrm{C} & -0.17525900 & 0.01896700 & 0.00006100 \\ \mathrm{C} & 0.52939400 & -1.19468000 & 0.00005200 \\ \mathrm{C} & 1.92239700 & -1.21989200 & 0.00000300 \\ \mathrm{C} & 2.63961100 & -0.02304800 & -0.00004600 \\ \mathrm{H} & 2.51418400 & 2.12795900 & -0.00008300 \\ \mathrm{H} & 0.06208800 & 2.17419700 & -0.00000200 \\ \mathrm{H} & -0.02866600 & -2.12676000 & 0.00009300 \\ \mathrm{H} & 2.44994800 & -2.16896700 & 0.00000200 \\ \mathrm{~B} & -1.74588600 & 0.00112100 & 0.00014000 \\ \mathrm{O} & -2.37439300 & -1.21249000 & -0.00025800 \\ \mathrm{H} & -3.33611900 & -1.13146100 & -0.00031100 \\ \mathrm{O} & -2.53184200 & 1.12322500 & 0.00014700 \\ \mathrm{H} & -2.05806200 & 1.96082000 & 0.00037500\end{array}$

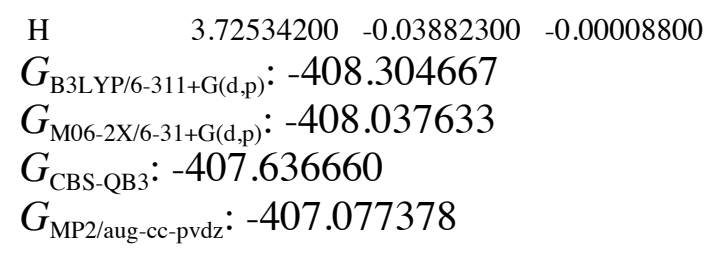

4 (4-fluorophenylboronic acid) exo-endo

$\begin{array}{lrrr}\mathrm{C} & 1.51433500 & 1.21649100 & 0.00006400 \\ \mathrm{C} & 0.12136300 & 1.21658700 & 0.00013500 \\ \mathrm{C} & -0.61623700 & 0.02397500 & 0.00010000 \\ \mathrm{C} & 0.09317700 & -1.18742800 & 0.00003000 \\ \mathrm{C} & 1.48506700 & -1.21862700 & -0.00001700 \\ \mathrm{C} & 2.16230600 & -0.00870600 & -0.00002200 \\ \mathrm{H} & 2.09228800 & 2.13391400 & 0.00007500 \\ \mathrm{H} & -0.38239100 & 2.18065400 & 0.00020200 \\ \mathrm{H} & -0.45925400 & -2.12232300 & 0.00002900 \\ \mathrm{H} & 2.04248300 & -2.14890800 & -0.00008000 \\ \mathrm{~B} & -2.18582700 & -0.00275500 & -0.00001800 \\ \mathrm{O} & -2.80243200 & -1.22209700 & 0.00006500 \\ \mathrm{H} & -3.76503100 & -1.15180000 & 0.00000300 \\ \mathrm{O} & -2.98040800 & 1.11257000 & -0.00022000 \\ \mathrm{H} & -2.51804700 & 1.95632500 & 0.00025000 \\ \mathrm{~F} & 3.51352600 & -0.02351300 & -0.00009900 \\ G_{\mathrm{B} 3 \mathrm{LYP} / 6-311+\mathrm{G}(\mathrm{d}, \mathrm{p})} & :-507.589108 & \\ G_{\mathrm{M} 06-2 \mathrm{X} / 6-31+\mathrm{G}(\mathrm{d}, \mathrm{p})} & :-507.263852 & \\ G_{\mathrm{CBS}-\mathrm{QB} 3}:-507.263852 & \\ G_{\mathrm{MP} 2 / \mathrm{aug}-\mathrm{cc}-\mathrm{pvdz}}:-506.14896 & \end{array}$




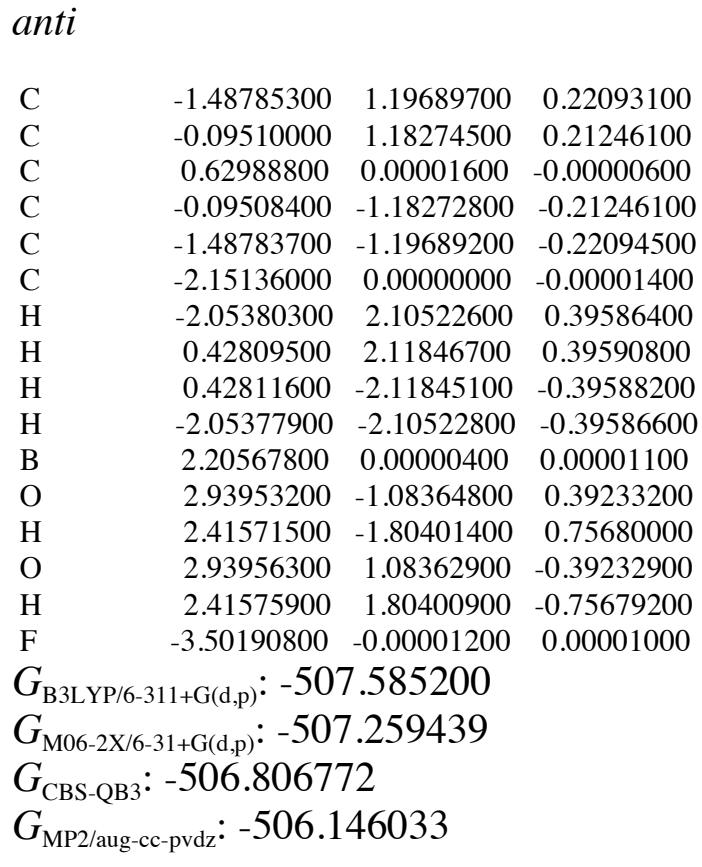

5 (4-methoxycarbonylphenylboronic acid)

exo-endo

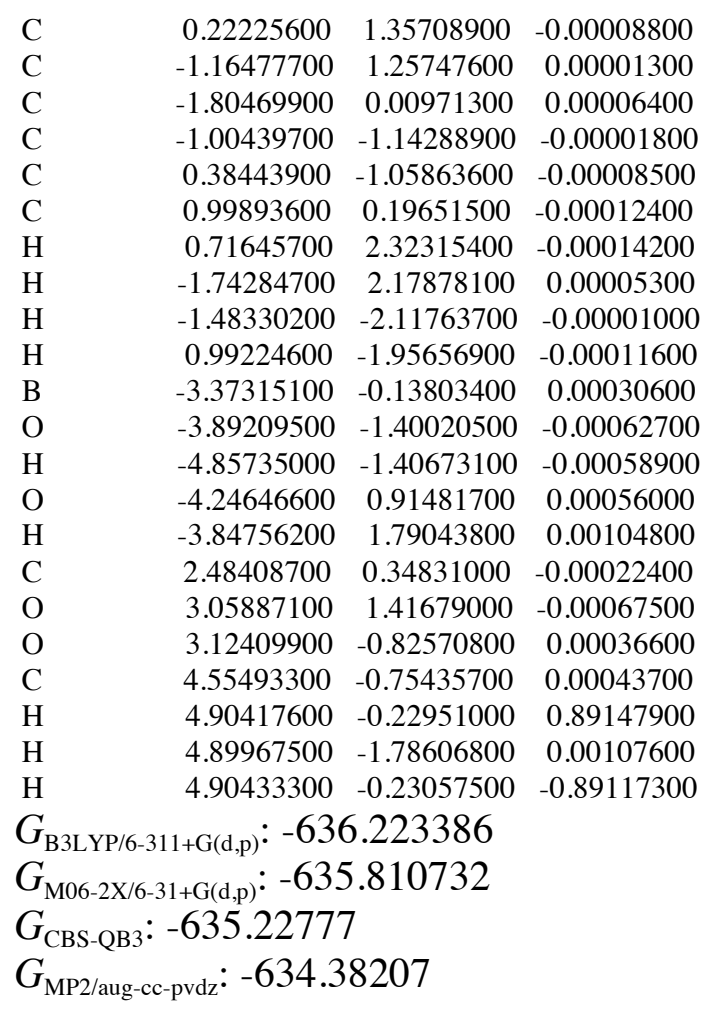

syn

$\begin{array}{lrrr}\mathrm{C} & 0.21458000 & 1.34962600 & -0.00007600 \\ \mathrm{C} & -1.17206500 & 1.24324700 & -0.00012000 \\ \mathrm{C} & -1.79891900 & -0.01110800 & -0.00016100 \\ \mathrm{C} & -0.99571300 & -1.16030000 & -0.00014200 \\ \mathrm{C} & 0.39283400 & -1.06716900 & -0.00009600 \\ \mathrm{C} & 0.99854600 & 0.19272900 & -0.00005700 \\ \mathrm{H} & 0.70491300 & 2.31784600 & -0.00005600 \\ \mathrm{H} & -1.78189000 & 2.14209200 & -0.00014400 \\ \mathrm{H} & -1.46930300 & -2.13799600 & -0.00018100 \\ \mathrm{H} & 1.00722900 & -1.96064000 & -0.00008300 \\ \mathrm{~B} & -3.36585100 & -0.12761600 & -0.00027900 \\ \mathrm{O} & -3.90829900 & -1.38329900 & 0.00006000 \\ \mathrm{H} & -4.87021600 & -1.43757400 & 0.00049200 \\ \mathrm{O} & -4.09004800 & 1.03217700 & 0.00028200 \\ \mathrm{H} & -5.04937100 & 0.94101300 & 0.00054100 \\ \mathrm{C} & 2.48222600 & 0.35187100 & 0.00000200 \\ \mathrm{O} & 3.05380300 & 1.42234400 & -0.00001600 \\ \mathrm{O} & 3.12950000 & -0.81941400 & 0.00008800 \\ \mathrm{C} & 4.55943100 & -0.73971000 & 0.00015100 \\ \mathrm{H} & 4.90614300 & -0.21340400 & 0.89135800 \\ \mathrm{H} & 4.91036100 & -1.76940000 & 0.00018200 \\ \mathrm{H} & 4.90622600 & -0.21342600 & -0.89103600 \\ G_{\text {B3LYP/6-311+G(d,p) }}:-636.221365 & \\ G_{\text {M06-2X/6-31+G(d,p) }}:-635.809220 & \\ G_{\mathrm{CBS}-\mathrm{QB} 3}:-635.225412 & \\ G_{\mathrm{MP} 2 / \text { aug-cc-pvdz }}:-634.380506 & \end{array}$

anti

$\begin{array}{llll}\text { C } & 0.20518200 & 1.33274000 & -0.18468700\end{array}$

$\begin{array}{lllll}\mathrm{C} & & -1.18122300 & 1.22706900 & -0.16767600\end{array}$

$\begin{array}{lllll}\text { C } & -1.81437100 & -0.01468100 & -0.00323700\end{array}$

$\begin{array}{llll}\text { C } & -1.00589700 & -1.15102000 & 0.14872700\end{array}$

$\begin{array}{llll}\text { C } & 0.38282900 & -1.05866400 & 0.14372700\end{array}$

$\begin{array}{llll}\text { C } & 0.98941900 & 0.18846600 & -0.02591800\end{array}$

$\begin{array}{llll}\mathrm{H} & 0.69303500 & 2.29240900 & -0.32068600\end{array}$

$\mathrm{H} \quad-1.77355300 \quad 2.12915000 \quad-0.30340700$

$\begin{array}{llll}\mathrm{H} & -1.46141600 & -2.12815300 & 0.29289000\end{array}$

$\begin{array}{llll}\mathrm{H} & 0.99523100 & -1.94445000 & 0.27157200\end{array}$

$\begin{array}{llll}\text { B } & -3.39043000 & -0.12890300 & 0.01397200\end{array}$

$\begin{array}{llll}\mathrm{O} & -4.04785600 & -1.23723600 & -0.43564700\end{array}$

$\begin{array}{lllll}\mathrm{H} & -3.47885500 & -1.89863100 & -0.84225800\end{array}$

$\begin{array}{llll}\mathrm{O} & -4.19007300 & 0.87342500 & 0.48200100\end{array}$

$\begin{array}{llll}\mathrm{H} & -3.71254200 & 1.61412200 & 0.86905800\end{array}$

$\begin{array}{llll}\text { C } & 2.47368700 & 0.34834700 & -0.04327200\end{array}$

$\begin{array}{llll}\mathrm{O} & 3.04025200 & 1.41270100 & -0.17608700\end{array}$

$\begin{array}{llll}\mathrm{O} & 3.12095700 & -0.81197900 & 0.10434000\end{array}$

$\begin{array}{llll}\text { C } & 4.55147900 & -0.73032400 & 0.10594200\end{array}$

$\begin{array}{llll}\mathrm{H} & 4.88934100 & -0.10201900 & 0.93202500\end{array}$

$\begin{array}{llll}\mathrm{H} & 4.90291900 & -1.75217600 & 0.23076000\end{array}$

$\mathrm{H} \quad 4.90512200 \quad-0.31262300 \quad-0.83830100$

$G_{\mathrm{B} 3 \mathrm{LYP} / 6-311+\mathrm{G}(\mathrm{d}, \mathrm{p})}:-636.219828$

$G_{\mathrm{M} 06-2 \mathrm{X} / 6-31+\mathrm{G}(\mathrm{d}, \mathrm{p})}:-635.806454$

$G_{\mathrm{CBS}-\mathrm{QB} 3}:-635.224273$

$G_{\mathrm{MP} 2 / \text { aug-cc-pvdz }}:-634.379123$

$\mathrm{S} 36$ 


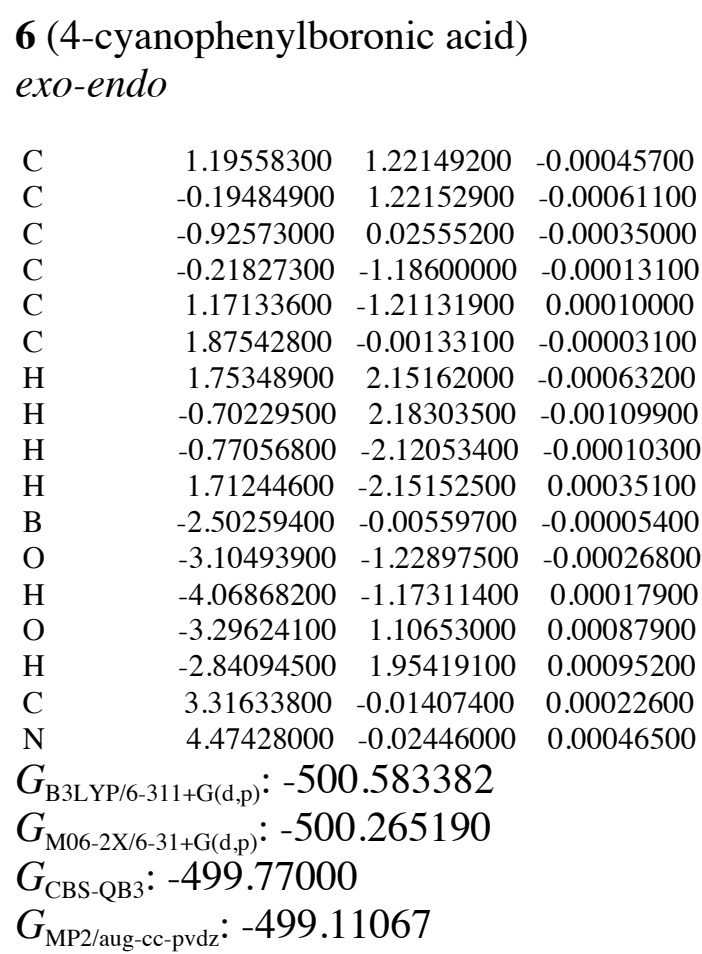

$\begin{array}{lrrr}\text { syn } & & & \\ \mathrm{C} & 1.18376700 & -1.21718400 & -0.03206800 \\ \mathrm{C} & -0.20652200 & -1.20566400 & -0.02883700 \\ \mathrm{C} & -0.92089100 & 0.00012100 & -0.00002300 \\ \mathrm{C} & -0.20646000 & 1.20583100 & 0.02882900 \\ \mathrm{C} & 1.18385000 & 1.21726500 & 0.03205400 \\ \mathrm{C} & 1.87501000 & 0.00002900 & -0.00001300 \\ \mathrm{H} & 1.73523100 & -2.15106900 & -0.05838400 \\ \mathrm{H} & -0.74986400 & -2.14561000 & -0.05101200 \\ \mathrm{H} & -0.74972300 & 2.14583000 & 0.05107900 \\ \mathrm{H} & 1.73534900 & 2.15112700 & 0.05841500 \\ \mathrm{~B} & -2.49377800 & 0.00002700 & -0.00001300 \\ \mathrm{O} & -3.12473700 & 1.21029300 & -0.06039600 \\ \mathrm{H} & -4.08806000 & 1.19492000 & -0.07776900 \\ \mathrm{O} & -3.12437300 & -1.21048400 & 0.06042400 \\ \mathrm{H} & -4.08770200 & -1.19531500 & 0.07776100 \\ \mathrm{C} & 3.31589800 & -0.00003600 & 0.00000600 \\ \mathrm{~N} & 4.47408900 & -0.00009400 & 0.00000800 \\ G_{\mathrm{B} 3 \mathrm{LYP} / 6-311+\mathrm{G}(\mathrm{d}, \mathrm{p})}:-500.581987 & \\ G_{\mathrm{M} 06-2 \mathrm{X} / 6-31+\mathrm{G}(\mathrm{d}, \mathrm{p})}:-500.264104 & \\ G_{\mathrm{CBS}-\mathrm{QB} 3}:-499.768665 & \\ G_{\mathrm{MP} 2 / \text { aug-cc-pvdz }}:-499.109563 & \end{array}$

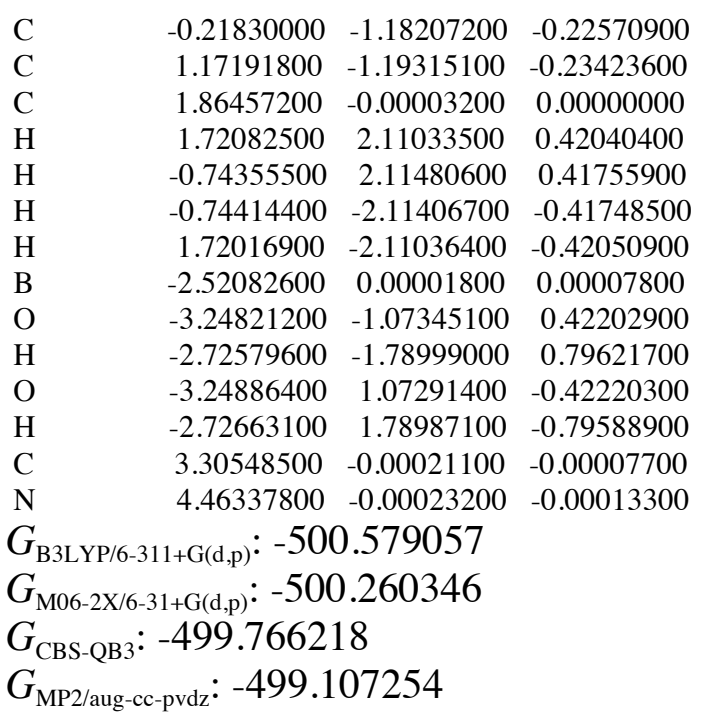

7 (Catechol) for simplicity and computational cost the 4-tert-butyl substituent was not included

$\begin{array}{lrrr}\mathrm{C} & -1.90960600 & -0.65869100 & -0.00000600 \\ \mathrm{C} & -1.87667800 & 0.73335600 & 0.00000100 \\ \mathrm{C} & -0.65019900 & 1.40264900 & 0.00000300 \\ \mathrm{C} & 0.53169500 & 0.67414300 & 0.00000100 \\ \mathrm{C} & 0.50046100 & -0.72691200 & 0.00000000 \\ \mathrm{C} & -0.71973400 & -1.38977300 & -0.00000600 \\ \mathrm{H} & -2.85932500 & -1.18315700 & -0.00001100 \\ \mathrm{H} & -2.79795800 & 1.30589700 & 0.00000200 \\ \mathrm{H} & -0.61151300 & 2.48879800 & 0.00000500 \\ \mathrm{H} & -0.72151900 & -2.47508400 & -0.00000800 \\ \mathrm{O} & 1.66146300 & -1.43802100 & 0.00000000 \\ \mathrm{H} & 2.40670200 & -0.81995800 & 0.00003400 \\ \mathrm{O} & 1.78662900 & 1.22389700 & -0.00000900 \\ \mathrm{H} & 1.74325200 & 2.18786500 & 0.00009300 \\ G_{\mathrm{B} 3 \mathrm{LYP} / 6-311+\mathrm{G}(\mathrm{d}, \mathrm{p})}:-382.735204 & \\ G_{\mathrm{M} 06-2 \mathrm{X} / 6-31+\mathrm{G}(\mathrm{d}, \mathrm{p})}:-382.483542 & \\ G_{\mathrm{CBS}-\mathrm{QB} 3}:-382.126923 & \\ G_{\mathrm{MP} 2 / \mathrm{aug}-\mathrm{cc}-\mathrm{pvdz} \mathrm{C}}:-381.610814 & \end{array}$

8 (ortho-phenylenediamine) for simplicity and computational cost the 4-tert-butyl substituent was not included

$\begin{array}{lrrr}\mathrm{C} & 1.92227700 & -0.69583400 & -0.01855000 \\ \mathrm{C} & 1.92228100 & 0.69582300 & 0.01856800 \\ \mathrm{C} & 0.71021400 & 1.38862300 & 0.02490300 \\ \mathrm{C} & -0.50475700 & 0.70534600 & 0.00023600 \\ \mathrm{C} & -0.50476200 & -0.70534100 & -0.00024000 \\ \mathrm{C} & 0.71020500 & -1.38862500 & -0.02489000 \\ \mathrm{H} & 2.85694700 & -1.24705300 & -0.03311300 \\ \mathrm{H} & 0.70220900 & 2.47578800 & 0.03640500 \\ \mathrm{H} & 0.70219400 & -2.47579000 & -0.03639000\end{array}$




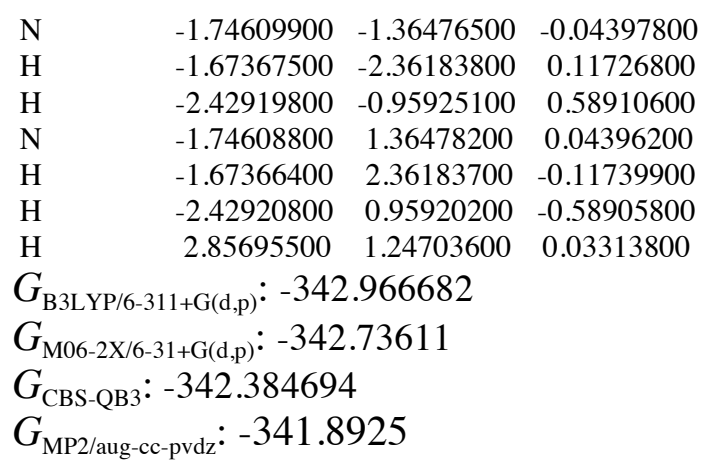

\section{9 (2-hydroxybenzenethiol)}

$\begin{array}{lrrr}\mathrm{C} & -2.37169700 & -0.01858100 & 0.01244900 \\ \mathrm{C} & -1.82905700 & -1.30600400 & 0.00077600 \\ \mathrm{C} & -0.44713500 & -1.46383400 & -0.01351000 \\ \mathrm{C} & 0.39770800 & -0.35088900 & -0.00361500 \\ \mathrm{C} & -0.15653800 & 0.93863100 & -0.00252900 \\ \mathrm{C} & -1.54507700 & 1.09793000 & 0.00875200 \\ \mathrm{H} & -3.44859900 & 0.11761900 & 0.02015000 \\ \mathrm{H} & -2.47544200 & -2.17673500 & -0.00177700 \\ \mathrm{H} & -0.00538700 & -2.45548600 & -0.03130700 \\ \mathrm{H} & -1.95075700 & 2.10420400 & 0.01245800 \\ \mathrm{O} & 0.60460500 & 2.05877100 & -0.00340300 \\ \mathrm{H} & 1.54029600 & 1.80351900 & -0.05716300 \\ \mathrm{~S} & 2.17606200 & -0.54044800 & -0.07376800 \\ \mathrm{H} & 2.39682100 & -0.59964300 & 1.25120200 \\ G_{\mathrm{B} 3 \mathrm{LYP} / 6-311+\mathrm{G}(\mathrm{d}, \mathrm{p})} & :-705.708788 & \\ G_{\mathrm{M} 06-2 \mathrm{X} / 6-31+\mathrm{G}(\mathrm{d}, \mathrm{p})} & :-705.442508 & \\ G_{\mathrm{CBS}-\mathrm{QB} 3}:-704.732877 & \\ G_{\mathrm{MP} 2 / \text { aug-cc-pvdz }}:-704.223323 & \end{array}$

\section{0 toluene-3,4-dithiol}

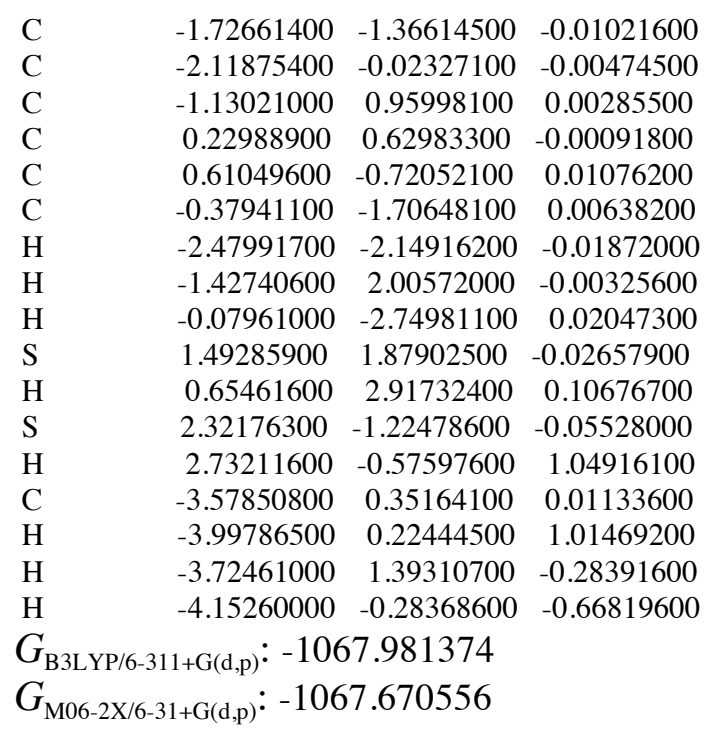

$G_{\text {CBS-QB3 }}:-1027.335774$

$G_{\mathrm{MP} 2 / \text { aug-cc-pvdz }}:-1026.831643$

\section{$11 a$}

\begin{tabular}{|c|c|c|c|}
\hline $\mathrm{C}$ & -3.06557900 & -0.99825700 & -0.00004300 \\
\hline $\mathrm{C}$ & -1.67236700 & -1.04035600 & -0.00003600 \\
\hline $\mathrm{C}$ & -0.89925200 & 0.12666900 & -0.00000500 \\
\hline $\mathrm{C}$ & -1.57216000 & 1.36257700 & 0.00002300 \\
\hline $\mathrm{C}$ & -2.95512800 & 1.42725500 & 0.00001800 \\
\hline $\mathrm{C}$ & -3.70896700 & 0.24394000 & -0.00001700 \\
\hline $\mathrm{H}$ & -3.63035700 & -1.92255700 & -0.00006700 \\
\hline $\mathrm{H}$ & -1.17875700 & -2.00894100 & -0.00005700 \\
\hline $\mathrm{H}$ & -0.99781200 & 2.28526100 & 0.00004900 \\
\hline $\mathrm{H}$ & -3.48035400 & 2.37679100 & 0.00004600 \\
\hline B & 0.63782400 & 0.05936600 & 0.00001100 \\
\hline $\mathrm{O}$ & 1.37770000 & -1.11979000 & -0.00002400 \\
\hline $\mathrm{O}$ & 1.47901300 & 1.16842500 & 0.00001200 \\
\hline $\mathrm{C}$ & 2.69495400 & -0.72906600 & -0.00001100 \\
\hline $\mathrm{C}$ & 2.75681400 & 0.66306900 & 0.00000900 \\
\hline $\mathrm{C}$ & 3.83129000 & -1.51443300 & -0.00002200 \\
\hline $\mathrm{C}$ & 3.95875200 & 1.34378900 & 0.00001900 \\
\hline $\mathrm{C}$ & 5.05763200 & -0.83684000 & -0.00000800 \\
\hline $\mathrm{H}$ & 3.77012800 & -2.59681000 & -0.00004000 \\
\hline $\mathrm{C}$ & 5.11990000 & 0.55909500 & 0.00001200 \\
\hline $\mathrm{H}$ & 3.99468200 & 2.42731700 & 0.00003400 \\
\hline $\mathrm{H}$ & 5.97793000 & -1.41123100 & -0.00001600 \\
\hline $\mathrm{H}$ & 6.08778800 & 1.04933600 & 0.00002200 \\
\hline $\mathrm{C}$ & -5.86266500 & -0.76560700 & 0.00005200 \\
\hline $\mathrm{H}$ & -6.89359700 & -0.41595700 & 0.00011200 \\
\hline $\mathrm{H}$ & -5.67851800 & -1.36827900 & -0.89559300 \\
\hline $\mathrm{H}$ & -5.67840500 & -1.36826100 & 0.89568600 \\
\hline 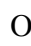 & -5.05436200 & 0.40205000 & -0.00001000 \\
\hline \multicolumn{4}{|c|}{$G_{\mathrm{B} 3 \mathrm{LYP} / 6-311+\mathrm{G}(\mathrm{d}, \mathrm{p})}:-752.660873$} \\
\hline \multirow{2}{*}{\multicolumn{4}{|c|}{$\begin{array}{l}G_{\mathrm{M} 06-2 \mathrm{X} / 6-31+\mathrm{G}(\mathrm{d}, \mathrm{p})}:-752.190222 \\
G_{\mathrm{CBS}-\mathrm{OB} 3}:-751.423423\end{array}$}} \\
\hline & & & \\
\hline \multicolumn{4}{|c|}{$G_{\mathrm{MP} 2 / \text { aug-cc-pvdz }}:-750.392568$} \\
\hline
\end{tabular}

\section{1b}

$\begin{array}{lrrr}\text { C } & -2.29812500 & 1.22904600 & -0.00005600 \\ \text { C } & -0.90356200 & 1.22131900 & -0.00008500 \\ \text { C } & -0.18662600 & 0.01957900 & -0.00000100 \\ \text { C } & -0.91610100 & -1.17990400 & 0.00012100 \\ \text { C } & -2.30489600 & -1.17016300 & 0.00015200 \\ \text { C } & -3.02739700 & 0.03449900 & 0.00005800 \\ \text { H } & -2.81288500 & 2.18329300 & -0.00013200 \\ \text { H } & -0.36656600 & 2.16661800 & -0.00017900 \\ \text { H } & -0.38735300 & -2.12976800 & 0.00019000 \\ \text { H } & -2.83555500 & -2.11831000 & 0.00025500 \\ \text { B } & 1.35564000 & 0.01210400 & -0.00004200 \\ \text { O } & 2.15231800 & 1.15218400 & -0.00009500 \\ \text { O } & 2.13877700 & -1.13731700 & -0.00002300 \\ \text { C } & 3.44887900 & 0.69641900 & -0.00011600 \\ \text { C } & 3.44060800 & -0.69699200 & -0.00007000\end{array}$




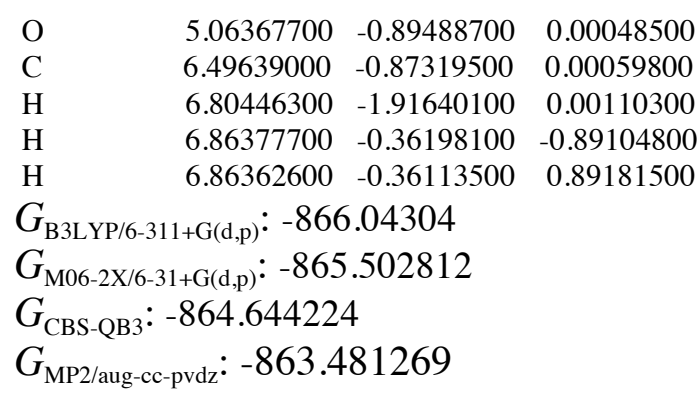

\section{$11 f$}

$\begin{array}{lrrr}\mathrm{C} & -3.17471100 & 1.21824600 & -0.00027200 \\ \mathrm{C} & -1.78515900 & 1.20889800 & -0.00010300 \\ \mathrm{C} & -1.07453600 & 0.00002400 & -0.00001400 \\ \mathrm{C} & -1.78514800 & -1.20885700 & -0.00010800 \\ \mathrm{C} & -3.17469800 & -1.21821500 & -0.00027700 \\ \mathrm{C} & -3.86478700 & 0.00001400 & -0.00036200 \\ \mathrm{H} & -3.72610200 & 2.15239900 & -0.00033500 \\ \mathrm{H} & -1.24476100 & 2.15107900 & -0.00002500 \\ \mathrm{H} & -1.24473700 & -2.15103100 & -0.00003500 \\ \mathrm{H} & -3.72608300 & -2.15237200 & -0.00034400 \\ \mathrm{~B} & 0.47568300 & 0.00002000 & 0.00021600 \\ \mathrm{O} & 1.25757200 & 1.14413900 & 0.00014200 \\ \mathrm{O} & 1.25755000 & -1.14411500 & 0.00012700 \\ \mathrm{C} & 2.55902000 & 0.69633800 & 0.00024700 \\ \mathrm{C} & 2.55900600 & -0.69634000 & 0.00024000 \\ \mathrm{C} & 3.72838900 & 1.43157900 & 0.00031000 \\ \mathrm{C} & 3.72836100 & -1.43160300 & 0.00029400 \\ \mathrm{C} & 4.92204700 & 0.69895900 & 0.00038500 \\ \mathrm{H} & 3.71568900 & 2.51552300 & 0.00030200 \\ \mathrm{C} & 4.92203300 & -0.69900600 & 0.00037700 \\ \mathrm{H} & 3.71564000 & -2.51554700 & 0.00027200 \\ \mathrm{H} & 5.86732800 & 1.23123100 & 0.00043800 \\ \mathrm{H} & 5.86730400 & -1.23129700 & 0.00042400 \\ \mathrm{C} & -5.30589200 & 0.00001400 & -0.00054800 \\ \mathrm{~N} & -6.46388900 & -0.00008400 & -0.00070800 \\ G_{\mathrm{B} 3 \mathrm{LYP} / 6-311+\mathrm{G}(\mathrm{d}, \mathrm{p})}:-730.402839 & \\ G_{\mathrm{M} 06-2 \mathrm{X} / 6-31+\mathrm{G}(\mathrm{d}, \mathrm{p})} & -729.95647 & \\ G_{\mathrm{CBS}-\mathrm{QB} 3}:-729.186577 & & \\ G_{\mathrm{MP} 2 / \mathrm{aug}-\mathrm{ccc}-\mathrm{pvdz}}:-728.209628 & \\ & & & \\ & & & \end{array}$

\section{$12 a$}

$\begin{array}{rrrr}\mathrm{C} & 3.13287900 & -0.98760600 & 0.12730500 \\ \mathrm{C} & 1.73766000 & -1.02771700 & 0.13034200 \\ \mathrm{C} & 0.95394800 & 0.12212000 & -0.01784500 \\ \mathrm{C} & 1.63725600 & 1.34267500 & -0.17376000 \\ \mathrm{C} & 3.02163100 & 1.41092100 & -0.18191800 \\ \mathrm{C} & 3.77851200 & 0.24154400 & -0.03070000 \\ \mathrm{H} & 3.69456400 & -1.90604700 & 0.24908600 \\ \mathrm{H} & 1.25796500 & -1.99494700 & 0.26225400 \\ \mathrm{H} & 1.07544000 & 2.26542600 & -0.30017900 \\ \mathrm{H} & 3.54194500 & 2.35523600 & -0.30632800\end{array}$

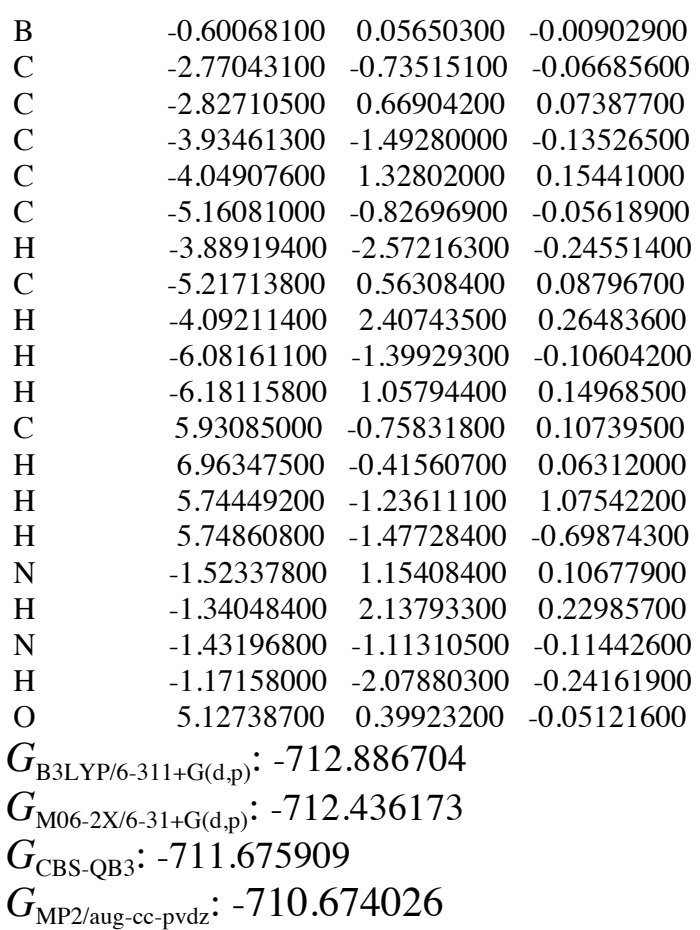

$12 b$

$\begin{array}{lrrr}\mathrm{C} & 2.35514000 & 1.21290100 & -0.18422900 \\ \mathrm{C} & 0.95949500 & 1.20183600 & -0.17813700 \\ \mathrm{C} & 0.23232100 & 0.02071900 & 0.01014800 \\ \mathrm{C} & 0.97348400 & -1.15754100 & 0.19644500 \\ \mathrm{C} & 2.36333100 & -1.15011700 & 0.19282300 \\ \mathrm{C} & 3.08823300 & 0.03633500 & 0.00039900 \\ \mathrm{H} & 2.86509500 & 2.15735400 & -0.33952700 \\ \mathrm{H} & 0.43434600 & 2.14158900 & -0.33553500 \\ \mathrm{H} & 0.45792400 & -2.10205900 & 0.35650600 \\ \mathrm{H} & 2.89256300 & -2.08737500 & 0.34372200 \\ \mathrm{~B} & -1.32604800 & 0.01223400 & 0.00863400 \\ \mathrm{C} & -3.52929900 & 0.70165200 & 0.07251400 \\ \mathrm{C} & -3.52047900 & -0.70330400 & -0.07060700 \\ \mathrm{C} & -4.72802600 & 1.40343200 & 0.14373400 \\ \mathrm{C} & -4.70999500 & -1.41950700 & -0.15173100 \\ \mathrm{C} & -5.92120800 & 0.68034800 & 0.06444300 \\ \mathrm{H} & -4.73376100 & 2.48345400 & 0.25631400 \\ \mathrm{C} & -5.91235900 & -0.71084700 & -0.08262100 \\ \mathrm{H} & -4.70171000 & -2.49951700 & -0.26433200 \\ \mathrm{H} & -6.86793700 & 1.20840800 & 0.11743600 \\ \mathrm{H} & -6.85230600 & -1.25004000 & -0.14343200 \\ \mathrm{C} & 4.61835800 & -0.00293300 & -0.00551600 \\ \mathrm{C} & 5.11998400 & -0.54982800 & 1.34306200 \\ \mathrm{C} & 5.09561000 & -0.92831500 & -1.13909700 \\ \mathrm{C} & 5.23335700 & 1.38376900 & -0.22317900 \\ \mathrm{H} & 4.78878900 & 0.08887700 & 2.16845400 \\ \mathrm{H} & 4.75250000 & -1.56338300 & 1.52884000 \\ \mathrm{H} & 6.21502400 & -0.58149400 & 1.34953700 \\ \mathrm{H} & 4.75059100 & -0.55932800 & -2.11048300 \\ \mathrm{H} & 6.19027500 & -0.96915700 & -1.15416500 \\ \mathrm{H} & 4.72110400 & -1.94813700 & -1.01027500 \\ & & & \\ & & & \end{array}$

S40 


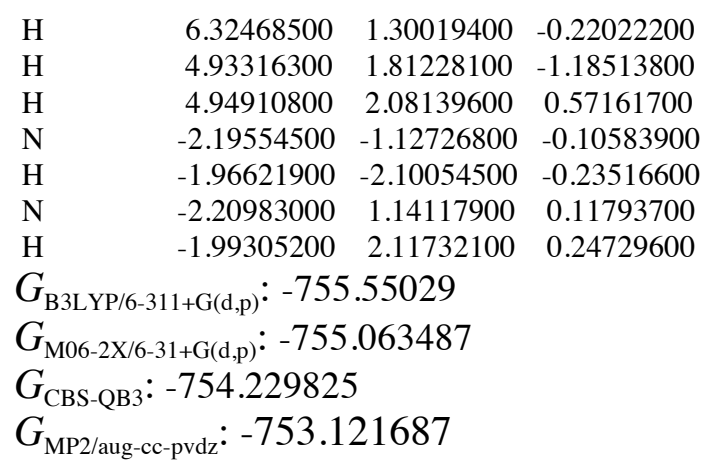

\section{$12 \mathrm{c}$}

$\begin{array}{lrrr}\mathrm{C} & 3.96384800 & 1.20236100 & -0.09855800 \\ \mathrm{C} & 2.57029300 & 1.19701300 & -0.09793800 \\ \mathrm{C} & 1.84376300 & -0.00000600 & -0.00000100 \\ \mathrm{C} & 2.57029900 & -1.19701300 & 0.09794400 \\ \mathrm{C} & 3.96386100 & -1.20235300 & 0.09856500 \\ \mathrm{C} & 4.66424200 & 0.00000300 & -0.00000100 \\ \mathrm{H} & 4.50313900 & 2.14155800 & -0.17706500 \\ \mathrm{H} & 2.04257700 & 2.14450600 & -0.18080600 \\ \mathrm{H} & 2.04259800 & -2.14451500 & 0.18081500 \\ \mathrm{H} & 4.50314700 & -2.14155300 & 0.17707500 \\ \mathrm{~B} & 0.28344200 & -0.00000600 & -0.00000100 \\ \mathrm{C} & -1.91483300 & 0.70493000 & 0.03823100 \\ \mathrm{C} & -1.91483600 & -0.70493200 & -0.03823500 \\ \mathrm{C} & -3.10894900 & 1.41704500 & 0.07904200 \\ \mathrm{C} & -3.10895500 & -1.41704300 & -0.07904000 \\ \mathrm{C} & -4.30657300 & 0.69843100 & 0.03927600 \\ \mathrm{H} & -3.10760800 & 2.50125600 & 0.13919600 \\ \mathrm{C} & -4.30657600 & -0.69842400 & -0.03926300 \\ \mathrm{H} & -3.10761900 & -2.50125300 & -0.13919600 \\ \mathrm{H} & -5.24982600 & 1.23435000 & 0.06984200 \\ \mathrm{H} & -5.24983100 & -1.23433900 & -0.06982300 \\ \mathrm{~N} & -0.59256200 & -1.13815100 & -0.05974000 \\ \mathrm{H} & -0.37073000 & -2.11927800 & -0.12877300 \\ \mathrm{~N} & -0.59255800 & 1.13814300 & 0.05972000 \\ \mathrm{H} & -0.37071800 & 2.11926900 & 0.12874900 \\ \mathrm{H} & 5.75000100 & 0.00001500 & -0.00000100 \\ G_{\mathrm{B} 3 L Y \mathrm{P} / 6-311+\mathrm{G}(\mathrm{d}, \mathrm{p})} & :-598.356892 & \\ G_{\mathrm{M} 06-2 \mathrm{X} / 6-31+\mathrm{G}(\mathrm{d}, \mathrm{p})}:-597.981432 & \\ G_{\mathrm{CBS}-\mathrm{QB} 3}:-597.309583 & & \\ G_{\mathrm{MP} 2 / \mathrm{aug}-\mathrm{cc}-\mathrm{pvdz}}:-596.461327 & \\ & & & \\ & & -500 & \\ & & & \end{array}$

\section{2d}

$\begin{array}{lrrr}\mathrm{C} & 3.52738600 & 1.21404100 & -0.07781000 \\ \mathrm{C} & 2.13460600 & 1.19839500 & -0.07756300 \\ \mathrm{C} & 1.40761200 & 0.00000000 & -0.00000100 \\ \mathrm{C} & 2.13460600 & -1.19839500 & 0.07756700 \\ \mathrm{C} & 3.52738500 & -1.21404100 & 0.07781000 \\ \mathrm{C} & 4.19159900 & 0.00000000 & -0.00000400 \\ \mathrm{H} & 4.09348000 & 2.13715700 & -0.13761900\end{array}$

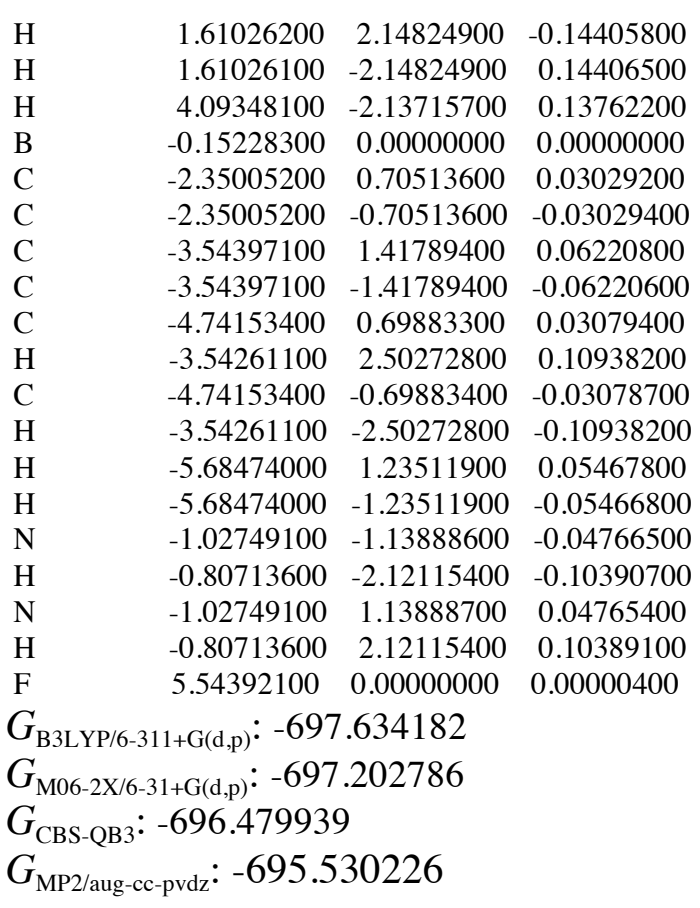

$12 \mathrm{e}$

$\begin{array}{rrr}2.29341700 & 1.36707900 & -0.18594700 \\ 0.90432300 & 1.31133300 & -0.18023500 \\ 0.22346100 & 0.09418000 & -0.01693300 \\ 0.99117300 & -1.07028800 & 0.14036700 \\ 2.38174700 & -1.02936500 & 0.13668200 \\ 3.03622400 & 0.19470200 & -0.02680100 \\ 2.81590200 & 2.30958400 & -0.31454700 \\ 0.34307900 & 2.23256100 & -0.31443600 \\ 0.49781900 & -2.02892800 & 0.27864400 \\ 2.96005900 & -1.93812400 & 0.26225000 \\ -1.33805300 & 0.03760900 & -0.00872400 \\ -3.55670600 & 0.65554700 & 0.10078900 \\ -3.50609200 & -0.74210600 & -0.09113600 \\ -4.77586600 & 1.31693400 & 0.20868200 \\ -4.67333200 & -1.49312600 & -0.18497000 \\ -5.94577700 & 0.55941700 & 0.11668000 \\ -4.81404200 & 2.39160500 & 0.35873300 \\ -5.89531800 & -0.82548100 & -0.07850900 \\ -4.63245800 & -2.56779400 & -0.33459700 \\ -6.90800100 & 1.05466600 & 0.19850000 \\ -6.81894200 & -1.39121200 & -0.14682900 \\ -2.16901300 & -1.12384000 & -0.15435600 \\ -1.91194900 & -2.08460400 & -0.32115400 \\ -2.25073300 & 1.13445000 & 0.14788500 \\ -2.06278600 & 2.11187100 & 0.31011300 \\ 4.52379000 & 0.29760200 & -0.03557300 \\ 5.13465800 & 1.33882600 & -0.15960700 \\ 5.12613300 & -0.88841200 & 0.10969200 \\ 6.55809500 & -0.86191900 & 0.11999700 \\ 6.91574000 & -0.24358000 & 0.94536000 \\ 6.86901600 & -1.89617500 & 0.25134200 \\ 6.93388100 & -0.46255600 & -0.82368600\end{array}$




$$
\begin{aligned}
& G_{\mathrm{B} 3 \mathrm{LYP} / 6-311+\mathrm{G}(\mathrm{d}, \mathrm{p})}:-826.271024 \\
& G_{\mathrm{M} 06-2 \mathrm{X} / 6-31+\mathrm{G}(\mathrm{d}, \mathrm{p})}:-825.750825 \\
& G_{\mathrm{CBS}-\mathrm{QB} 3}:-824.89752 \\
& G_{\mathrm{MP} 2 / \text { aug-cc-pvdz }}:-823.763837
\end{aligned}
$$

\begin{tabular}{|c|c|c|c|}
\hline $\mathrm{C}$ & -3.24280400 & -1.21251600 & -0.08534500 \\
\hline $\mathrm{C}$ & -1.85325700 & -1.19991400 & -0.08485100 \\
\hline $\mathrm{C}$ & -1.12960700 & 0.00000000 & 0.00000000 \\
\hline $\mathrm{C}$ & -1.85325700 & 1.19991400 & 0.08485100 \\
\hline $\mathrm{C}$ & -3.24280300 & 1.21251600 & 0.08534400 \\
\hline $\mathrm{C}$ & -3.93716800 & 0.00000000 & 0.00000000 \\
\hline $\mathrm{H}$ & -3.79084400 & -2.14639200 & -0.15195200 \\
\hline $\mathrm{H}$ & -1.32734200 & -2.14791300 & -0.15705200 \\
\hline $\mathrm{H}$ & -1.32734200 & 2.14791200 & 0.15705100 \\
\hline $\mathrm{H}$ & -3.79084300 & 2.14639200 & 0.15195100 \\
\hline B & 0.43423600 & 0.00000000 & 0.00000000 \\
\hline $\mathrm{C}$ & 2.62798400 & -0.70475100 & 0.03339500 \\
\hline $\mathrm{C}$ & 2.62798400 & 0.70475100 & -0.03339500 \\
\hline $\mathrm{C}$ & 3.82192100 & -1.41820900 & 0.06849300 \\
\hline $\mathrm{C}$ & 3.82192100 & 1.41820900 & -0.06849400 \\
\hline $\mathrm{C}$ & 5.01835200 & -0.69906100 & 0.03396300 \\
\hline $\mathrm{H}$ & 3.82044200 & -2.50274500 & 0.12047700 \\
\hline $\mathrm{C}$ & 5.01835200 & 0.69906100 & -0.03396600 \\
\hline $\mathrm{H}$ & 3.82044200 & 2.50274500 & -0.12047700 \\
\hline $\mathrm{H}$ & 5.96179700 & -1.23468900 & 0.06019500 \\
\hline $\mathrm{H}$ & 5.96179700 & 1.23468900 & -0.06019900 \\
\hline $\mathrm{N}$ & 1.30541600 & 1.13885800 & -0.05200400 \\
\hline $\mathrm{H}$ & 1.08529300 & 2.12108700 & -0.11235000 \\
\hline $\mathrm{N}$ & 1.30541600 & -1.13885800 & 0.05200900 \\
\hline $\mathrm{H}$ & 1.08529300 & -2.12108700 & 0.11235800 \\
\hline $\mathrm{C}$ & -5.37720600 & 0.00000000 & 0.00000000 \\
\hline $\mathrm{N}$ & -6.53545200 & 0.00000000 & -0.00000100 \\
\hline \multicolumn{4}{|c|}{$G_{\mathrm{B} 3 \mathrm{LYP} / 6-311+\mathrm{G}(\mathrm{d}, \mathrm{p})}:-690.631184$} \\
\hline \multicolumn{4}{|c|}{$G_{\mathrm{M} 06-2 \mathrm{X} / 6-31+\mathrm{G}(\mathrm{d}, \mathrm{p})}:-690.204925$} \\
\hline \multicolumn{4}{|c|}{$G_{\mathrm{CBS}-\mathrm{QB} 3}:-689.440406$} \\
\hline \multicolumn{4}{|c|}{$G_{\mathrm{MP} 2 / \text { aug-cc-pvdz }}:-688.492548$} \\
\hline
\end{tabular}

\section{$12 f$}

\section{3a}

$\begin{array}{lrrr}\mathrm{C} & -3.22409000 & 1.35208600 & -0.00010700 \\ \mathrm{C} & -1.84046000 & 1.37887400 & -0.00012000 \\ \mathrm{C} & -1.08203600 & 0.19248100 & -0.00001800 \\ \mathrm{C} & -1.77886300 & -1.02303300 & 0.00011600 \\ \mathrm{C} & -3.17161600 & -1.07461700 & 0.00013300 \\ \mathrm{C} & -3.89765600 & 0.12141400 & 0.00000900 \\ \mathrm{H} & -3.81033300 & 2.26505400 & -0.00018100 \\ \mathrm{H} & -1.33716200 & 2.34345700 & -0.00021800 \\ \mathrm{H} & -1.22137600 & -1.95599000 & 0.00020700 \\ \mathrm{H} & -3.67240200 & -2.03516600 & 0.00024400 \\ \mathrm{~B} & 0.45887100 & 0.20451700 & -0.00003200 \\ \mathrm{C} & 2.94379400 & 0.59966100 & -0.00010000 \\ \mathrm{C} & 2.55583100 & -0.74334600 & -0.00005200\end{array}$

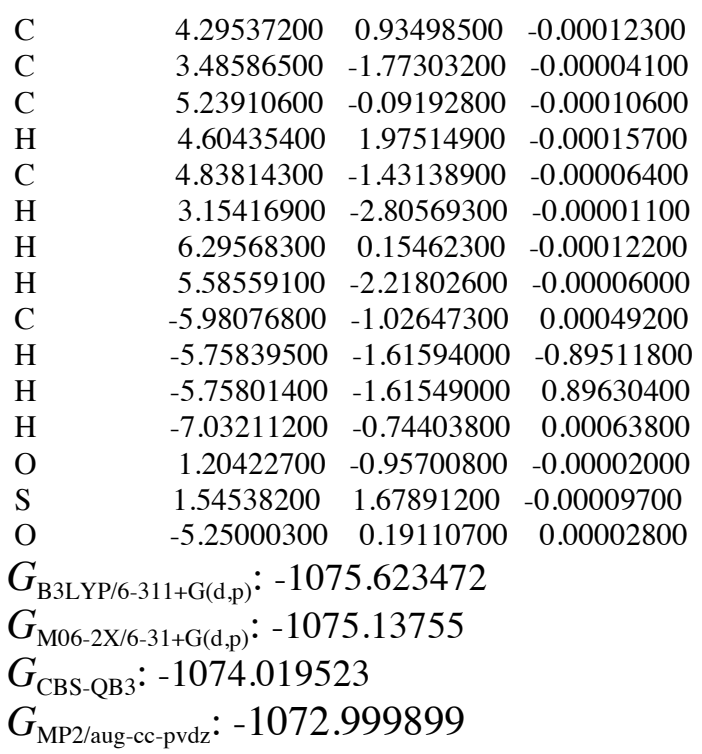

13b

$\begin{array}{lrrr}\mathrm{C} & -2.57331000 & -1.15317700 & 0.00002900 \\ \mathrm{C} & -1.18779900 & -1.24198200 & 0.00000600 \\ \mathrm{C} & -0.38635700 & -0.08857100 & 0.00001700 \\ \mathrm{C} & -1.03640600 & 1.15208600 & 0.00004800 \\ \mathrm{C} & -2.42783300 & 1.24081200 & 0.00007200 \\ \mathrm{C} & -3.22592200 & 0.09082000 & 0.00006500 \\ \mathrm{H} & -3.15631900 & -2.06989100 & 0.00001900 \\ \mathrm{H} & -0.72271600 & -2.22582300 & -0.00002300 \\ \mathrm{H} & -0.44566000 & 2.06444200 & 0.00005200 \\ \mathrm{H} & -2.88428600 & 2.22440500 & 0.00009500 \\ \mathrm{~B} & 1.15703900 & -0.15805400 & 0.00000100 \\ \mathrm{C} & 3.62573400 & -0.63899500 & -0.00006200 \\ \mathrm{C} & 3.28406700 & 0.71640700 & -0.00008600 \\ \mathrm{C} & 4.96564800 & -1.01897200 & -0.00009000 \\ \mathrm{C} & 4.24718400 & 1.71499600 & -0.00013900 \\ \mathrm{C} & 5.94261500 & -0.02384700 & -0.00014300 \\ \mathrm{H} & 5.24005000 & -2.06875800 & -0.00007200 \\ \mathrm{C} & 5.58687800 & 1.32866700 & -0.00016700 \\ \mathrm{H} & 3.94945700 & 2.75794600 & -0.00015900 \\ \mathrm{H} & 6.99037000 & -0.30554900 & -0.00016600 \\ \mathrm{H} & 6.36056100 & 2.08947200 & -0.00021000 \\ \mathrm{C} & -4.75532100 & 0.14091400 & 0.00009700 \\ \mathrm{C} & -5.28888800 & 1.57747200 & 0.00015700 \\ \mathrm{C} & -5.28630900 & -0.57310100 & -1.25579800 \\ \mathrm{C} & -5.28624900 & -0.57318800 & 1.25596900 \\ \mathrm{H} & -4.96588600 & 2.12943500 & 0.88895100 \\ \mathrm{H} & -4.96583200 & 2.12952800 & -0.88856000 \\ \mathrm{H} & -6.38305100 & 1.55520600 & 0.00012500 \\ \mathrm{H} & -4.98379300 & -1.62402600 & -1.28151800 \\ \mathrm{H} & -6.38106300 & -0.53723700 & -1.27012800 \\ \mathrm{H} & -4.91394900 & -0.08874700 & -2.16436800 \\ \mathrm{H} & -6.38100300 & -0.53733600 & 1.27034900 \\ \mathrm{H} & -4.98372000 & -1.62411200 & 1.28160300 \\ \mathrm{H} & -4.91385300 & -0.08889100 & 2.16455400 \\ \mathrm{O} & 1.94047800 & 0.97619800 & -0.00005900 \\ & & & \\ \mathrm{H} & & & \end{array}$

S42 


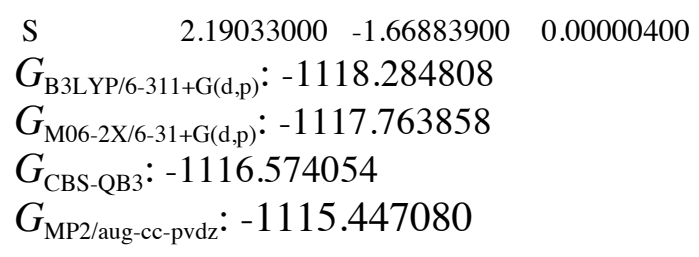

\section{$13 c$}

$\begin{array}{lrrr}\mathrm{C} & 2.57714600 & -1.16503500 & 0.00014100 \\ \mathrm{C} & 1.18865200 & -1.25122100 & -0.00007800 \\ \mathrm{C} & 0.38835900 & -0.09491000 & -0.00043000 \\ \mathrm{C} & 1.03485600 & 1.15519900 & -0.00049900 \\ \mathrm{C} & 2.42329200 & 1.24572700 & -0.00027600 \\ \mathrm{C} & 3.19683500 & 0.08497300 & 0.00004800 \\ \mathrm{H} & 3.17684000 & -2.06816600 & 0.00039200 \\ \mathrm{H} & 0.72228300 & -2.23128600 & 0.00000000 \\ \mathrm{H} & 0.43860800 & 2.06082600 & -0.00076300 \\ \mathrm{H} & 2.90344900 & 2.21781400 & -0.00035300 \\ \mathrm{~B} & -1.15417700 & -0.16616200 & -0.00076200 \\ \mathrm{C} & -3.63906300 & -0.63890500 & -0.00008300 \\ \mathrm{C} & -3.28877500 & 0.71570300 & -0.00001500 \\ \mathrm{C} & -4.98051000 & -1.01354700 & 0.00002300 \\ \mathrm{C} & -4.25002400 & 1.71633600 & 0.00017300 \\ \mathrm{C} & -5.95310300 & -0.01437300 & 0.00016300 \\ \mathrm{H} & -5.26125500 & -2.05969300 & 0.00004300 \\ \mathrm{C} & -5.59169700 & 1.33605400 & 0.00024000 \\ \mathrm{H} & -3.95085800 & 2.75714300 & 0.00021400 \\ \mathrm{H} & -7.00066500 & -0.29126500 & 0.00025800 \\ \mathrm{H} & -6.36080100 & 2.09916300 & 0.00036700 \\ \mathrm{O} & -1.94069000 & 0.96995700 & -0.00021700 \\ \mathrm{~S} & -2.20068900 & -1.67449700 & -0.00001500 \\ \mathrm{H} & 4.27899500 & 0.15364000 & 0.00022800 \\ G_{\mathrm{B} 3 L \mathrm{LP} / 6-311+\mathrm{G}(\mathrm{d}, \mathrm{p})} & -961.092561 & \\ G_{\mathrm{M} 06-2 \mathrm{X} / 6-31+\mathrm{G}(\mathrm{d}, \mathrm{p})}:-960.680937 & \\ G_{\mathrm{CBS}-\mathrm{QB} 3}:-959.652978 & & \\ G_{\mathrm{MP} 2 / \mathrm{aug}-\mathrm{cc}-\mathrm{pvdz}} & -9958.786619 & \\ & & & \\ & & & \end{array}$

\section{3d}

$\begin{array}{lrrr}\mathrm{C} & -3.71711700 & -1.07044800 & -0.00021200 \\ \mathrm{C} & -2.32962800 & -1.17418800 & -0.00018600 \\ \mathrm{C} & -1.51096700 & -0.03375100 & -0.00012000 \\ \mathrm{C} & -2.12596000 & 1.22910300 & -0.00007100 \\ \mathrm{C} & -3.51144800 & 1.35930300 & -0.00009400 \\ \mathrm{C} & -4.27344500 & 0.20020800 & -0.00016600 \\ \mathrm{H} & -4.36022800 & -1.94325100 & -0.00026200 \\ \mathrm{H} & -1.88139100 & -2.16500300 & -0.00022800 \\ \mathrm{H} & -1.50960800 & 2.12348700 & -0.00002100 \\ \mathrm{H} & -3.99976000 & 2.32752900 & -0.00005700 \\ \mathrm{~B} & 0.03243000 & -0.13706500 & -0.00009600 \\ \mathrm{C} & 2.48975500 & -0.66141300 & 0.00008800 \\ \mathrm{C} & 2.17265600 & 0.69963600 & 0.00011200 \\ \mathrm{C} & 3.82220400 & -1.06626600 & 0.00015600 \\ \mathrm{C} & 3.15318000 & 1.68084500 & 0.00020700\end{array}$

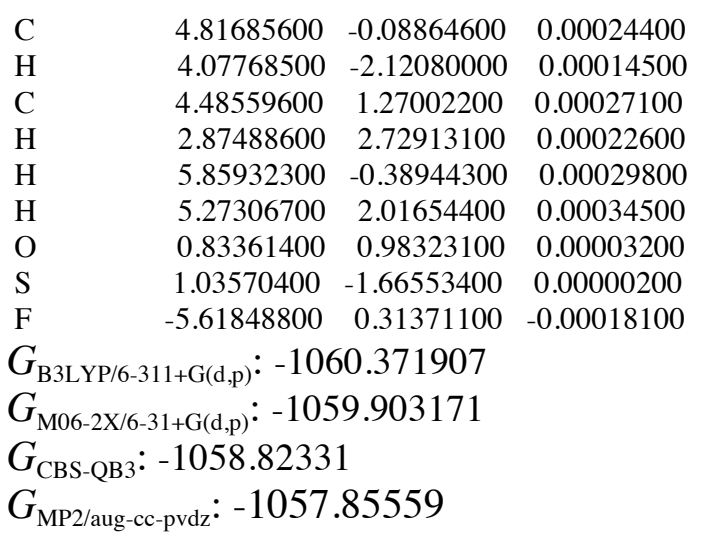

$13 e$

\begin{tabular}{|c|c|c|c|}
\hline $\mathrm{C}$ & 2.59177300 & -1.03616300 & -0.00007600 \\
\hline $\mathrm{C}$ & 1.20640400 & -1.15784900 & 0.00001700 \\
\hline & 0.38040000 & -0.02375400 & -0.00012400 \\
\hline & 0.98179400 & 1.24524200 & -0.00037600 \\
\hline & 2.36518700 & 1.37725000 & -0.00049400 \\
\hline & 3.17109300 & 0.23591300 & -0.00034200 \\
\hline & 3.22337400 & -1.91724300 & 0.00003800 \\
\hline & 0.76449300 & -2.15135900 & 0.00021900 \\
\hline & 0.35649200 & 2.13316800 & -0.00048000 \\
\hline & 2.83609300 & 2.35482600 & -0.00070700 \\
\hline 3 & -1.16724700 & -0.14031800 & -0.00001500 \\
\hline & -3.61774800 & -0.68197300 & 0.00011600 \\
\hline & -3.31093100 & 0.68153600 & 0.00013500 \\
\hline & -4.94764800 & -1.09621800 & 0.00018600 \\
\hline & -4.29784400 & 1.65639100 & 0.00023300 \\
\hline & -5.94835600 & -0.12547100 & 0.00028100 \\
\hline & -5.19570400 & -2.15244900 & 0.00016600 \\
\hline & -5.62671200 & 1.23597600 & 0.00030600 \\
\hline & -4.02626000 & 2.70635800 & 0.00025200 \\
\hline $\mathrm{H}$ & -6.98869400 & -0.43321800 & 0.00033500 \\
\hline 1 & -6.41969000 & 1.97648700 & 0.00038500 \\
\hline J & -1.97352700 & 0.97424900 & 0.00005600 \\
\hline & -2.15614900 & -1.67466800 & -0.00000600 \\
\hline & 4.65227600 & 0.42719000 & -0.00047800 \\
\hline D & 5.19637400 & 1.51132500 & -0.00060000 \\
\hline & 5.32388300 & -0.72863000 & 0.00026700 \\
\hline & 6.75231800 & -0.61680800 & 0.00062200 \\
\hline & 7.12630800 & -1.63822200 & 0.00145600 \\
\hline & 7.08644000 & -0.08322300 & -0.89094900 \\
\hline & 7.08589500 & -0.08195600 & 0.89163500 \\
\hline \multicolumn{4}{|c|}{$G_{\mathrm{B} 3 \mathrm{LYP} / 6-311+\mathrm{G}(\mathrm{d}, \mathrm{p})}:-1189.00567$} \\
\hline \multirow{2}{*}{\multicolumn{4}{|c|}{$\begin{array}{l}G_{\mathrm{M} 06-2 \mathrm{X} / 6-31+\mathrm{G}(\mathrm{d}, \mathrm{p})}:-1188.449968 \\
G_{\mathrm{CBS}-\mathrm{QB} 3}:-1187.240435\end{array}$}} \\
\hline & & & \\
\hline \multicolumn{4}{|c|}{$G_{\mathrm{MP} 2 / \text { aug-cc-pvdz }}:-1186.088508$} \\
\hline
\end{tabular}




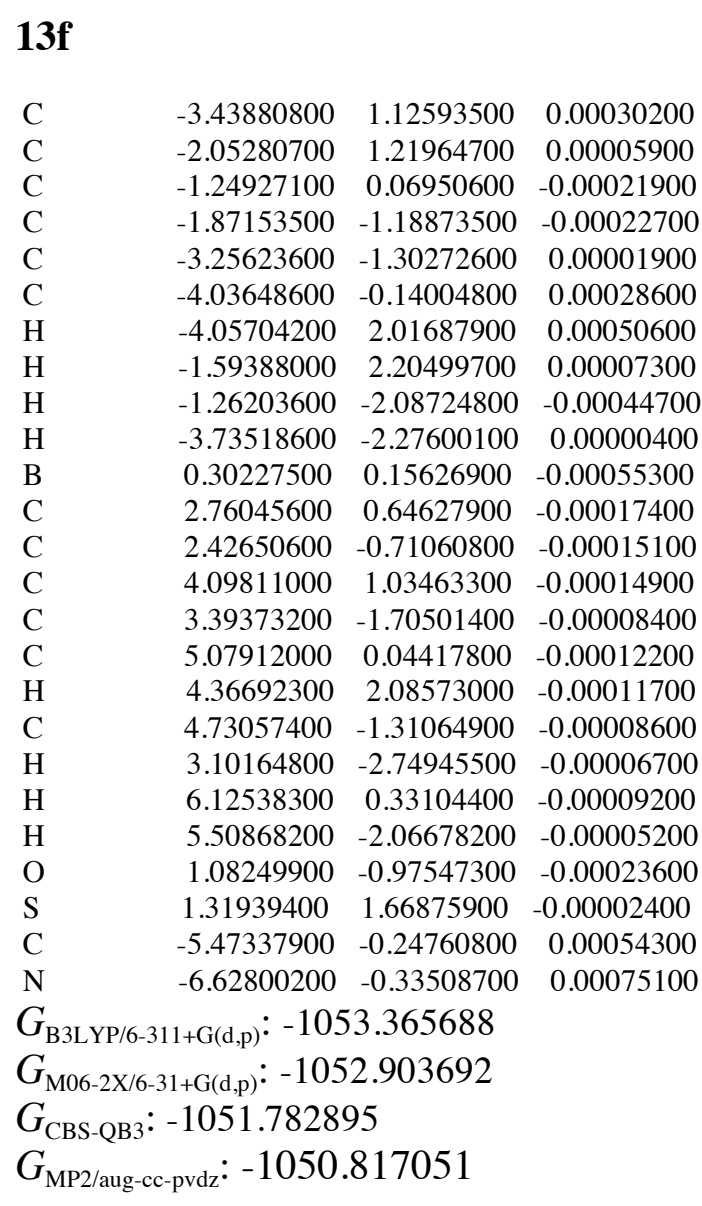

\section{$14 a$}

$\begin{array}{lrrr}\mathrm{C} & 3.67208600 & -1.48525900 & 0.03299300 \\ \mathrm{C} & 2.29347200 & -1.36903400 & 0.02960300 \\ \mathrm{C} & 1.65468400 & -0.11213100 & 0.00064100 \\ \mathrm{C} & 2.47996500 & 1.02160300 & -0.02509300 \\ \mathrm{C} & 3.86997400 & 0.93095900 & -0.02231200 \\ \mathrm{C} & 4.47037800 & -0.33198800 & 0.00733000 \\ \mathrm{H} & 4.15961100 & -2.45437300 & 0.05626900 \\ \mathrm{H} & 1.69707300 & -2.27825100 & 0.05035100 \\ \mathrm{H} & 2.03384400 & 2.01339200 & -0.04966900 \\ \mathrm{H} & 4.46457600 & 1.83605200 & -0.04252800 \\ \mathrm{~B} & 0.11421100 & 0.01171000 & -0.00067400 \\ \mathrm{C} & -2.50639300 & -0.48295300 & -0.00784700 \\ \mathrm{C} & -2.39436700 & 0.91149600 & 0.00794500 \\ \mathrm{C} & -3.76648700 & -1.08937700 & -0.01724000 \\ \mathrm{C} & -3.55061600 & 1.69804200 & 0.01527200 \\ \mathrm{C} & -4.92282300 & -0.31312900 & -0.00860900 \\ \mathrm{H} & -3.84028900 & -2.17359200 & -0.03152100 \\ \mathrm{C} & -4.79609600 & 1.08542400 & 0.00681600 \\ \mathrm{H} & -3.46982600 & 2.78064100 & 0.02634300 \\ \mathrm{H} & -5.69196600 & 1.70056500 & 0.01119300 \\ \mathrm{C} & 6.65914400 & 0.59735000 & -0.02054100 \\ \mathrm{H} & 6.49511100 & 1.18066600 & -0.93275700 \\ \mathrm{H} & 6.50008700 & 1.23156200 & 0.85799000 \\ \mathrm{H} & 7.67626100 & 0.20925200 & -0.01209000\end{array}$

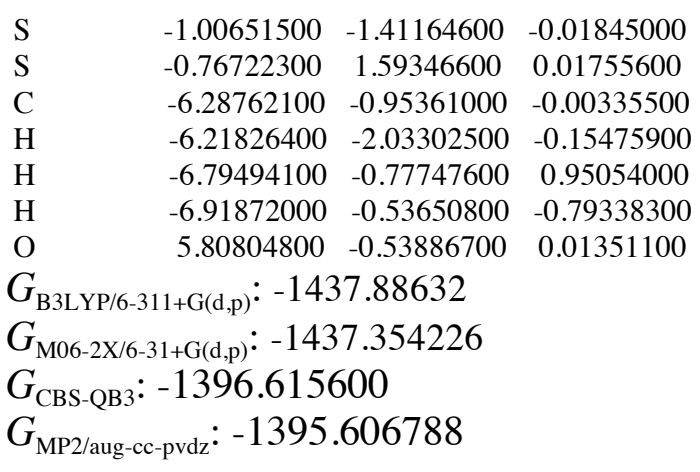

$14 b$

C $\quad-3.05143400 \quad-1.25514200 \quad-0.06205200$

$\begin{array}{llll}\text { C } & -1.65877000 & -1.19608900 & -0.05625200\end{array}$

$\begin{array}{llll}\mathrm{C} & -0.97457000 & 0.02546800 & 0.01327100\end{array}$

$\begin{array}{llll}\text { C } & -1.75687200 & 1.19266600 & 0.08014100\end{array}$

$\begin{array}{llll}\mathrm{C} & -3.14368500 & 1.13502100 & 0.07579600\end{array}$

$\begin{array}{lllll}\mathrm{C} & -3.82505500 & -0.09088300 & 0.00288900\end{array}$

$\mathrm{H} \quad-3.52779000 \quad-2.22741000 \quad-0.12086900$

$\mathrm{H} \quad-1.09948300 \quad-2.12770900 \quad-0.11114200$

$\begin{array}{llll}\mathrm{H} & -1.27400000 & 2.16574800 & 0.13986600\end{array}$

$\begin{array}{llll}\mathrm{H} & -3.70498600 & 2.06379200 & 0.12893100\end{array}$

$\begin{array}{llll}\text { B } & 0.57408900 & 0.08780000 & 0.01100400\end{array}$

$\begin{array}{llll}\text { C } & 3.17158000 & -0.50580400 & 0.01504800\end{array}$

$\begin{array}{lllll}\mathrm{C} & 3.11178000 & 0.89194400 & -0.01736200\end{array}$

$\begin{array}{llll}\text { C } & 4.40826400 & -1.15864600 & 0.02749000\end{array}$

$\begin{array}{lllll}\mathrm{C} & 4.29629600 & 1.63475500 & -0.03928400\end{array}$

$\begin{array}{llll}\mathrm{C} & 5.59241900 & -0.42604200 & 0.00460900\end{array}$

$\mathrm{H} \quad 4.44163300 \quad-2.24459300 \quad 0.05503400$

$\begin{array}{lllll}\mathrm{C} & 5.51794300 & 0.97624800 & -0.02791100\end{array}$

$\begin{array}{llll}\mathrm{H} & 4.25558500 & 2.71940600 & -0.06369100\end{array}$

$\begin{array}{llll}\mathrm{H} & 6.43609700 & 1.55741900 & -0.04345100\end{array}$

$\begin{array}{llll}\mathrm{C} & -5.35493000 & -0.10606900 & -0.00758100\end{array}$

$\begin{array}{llll}\text { C } & -5.87932900 & 0.55952400 & 1.27742000\end{array}$

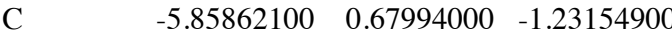

$\begin{array}{lllll}\mathrm{C} & -5.92063300 & -1.52838500 & -0.07917400\end{array}$

$\begin{array}{llll}\mathrm{H} & -5.53063600 & 0.02006200 & 2.16410200\end{array}$

$\mathrm{H} \quad \begin{array}{llll}\mathrm{H} & -5.54850500 & 1.59889200 & 1.35900000\end{array}$

$\begin{array}{llll}\mathrm{H} & -6.97469000 & 0.55358100 & 1.27932900\end{array}$

$\mathrm{H} \quad-5.49169400 \quad 0.22998700 \quad-2.15980100$

$\mathrm{H} \quad-6.95376000 \quad 0.67315300 \quad-1.25457800$

$\mathrm{H} \quad-\quad-5.52885400 \quad 1.72272500 \quad-1.20537800$

$\mathrm{H} \quad-7.01401800 \quad-1.48148200 \quad-0.08229200$

$\mathrm{H} \quad-5.60563700 \quad-2.04399300 \quad-0.99230900$

$\begin{array}{llll}\mathrm{H} & -5.61416400 & -2.12929300 & 0.78337600\end{array}$

$\begin{array}{llll}\mathrm{S} & 1.63728100 & -1.37606600 & 0.04473600\end{array}$

$\begin{array}{lllll}\mathrm{S} & 1.51132600 & 1.63357300 & -0.03047100\end{array}$

$\begin{array}{llll}\mathrm{C} & 6.93228700 & -1.11715400 & 0.00012200\end{array}$

$\begin{array}{llll}\mathrm{H} & 6.82537500 & -2.18810900 & 0.18575800\end{array}$

$\mathrm{H} \quad 7.43053700 \quad-0.98933100 \quad-0.96619300$

$\begin{array}{llll}\mathrm{H} & 7.59080600 & -0.70007600 & 0.76731700\end{array}$

$G_{\mathrm{B} 3 \mathrm{LYP} / 6-311+\mathrm{G}(\mathrm{d}, \mathrm{p})}:-1480.547$

$G_{\mathrm{M} 06-2 \mathrm{X} / 6-31+\mathrm{G}(\mathrm{d}, \mathrm{p})}:-1479.981181$

$G_{\mathrm{CBS}-\mathrm{QB} 3}:-1439.170126$

$G_{\mathrm{MP} / \text { aug-cc-pvdz: }}:-1438.053813$ 


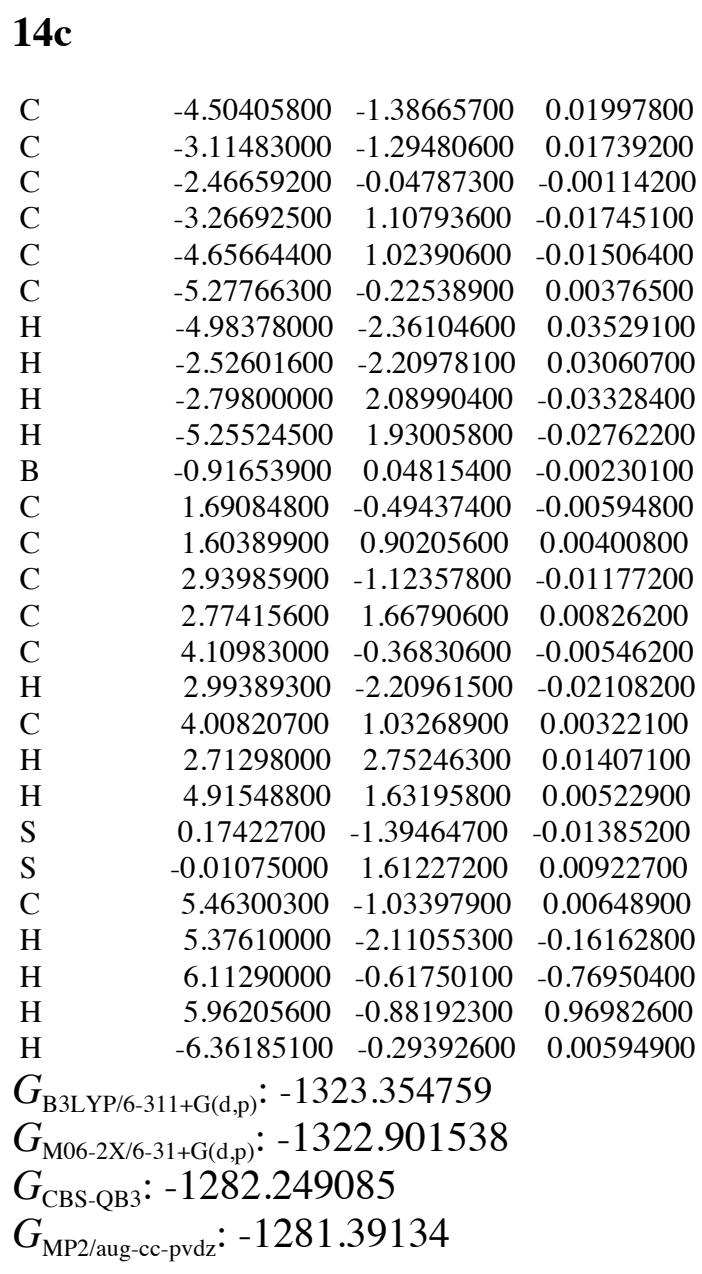

\section{4d}

$\begin{array}{lrrr}\text { C } & 4.12288700 & -1.33512400 & 0.00916000 \\ \mathrm{C} & 2.73446500 & -1.24897600 & 0.00795500 \\ \mathrm{C} & 2.07162800 & -0.00932800 & -0.00085100 \\ \mathrm{C} & 2.85940700 & 1.15552000 & -0.00869900 \\ \mathrm{C} & 4.24912400 & 1.09747600 & -0.00753600 \\ \mathrm{C} & 4.84829600 & -0.15312400 & 0.00142300 \\ \mathrm{H} & 4.64103300 & -2.28757800 & 0.01623000 \\ \mathrm{H} & 2.15918900 & -2.17130300 & 0.01435000 \\ \mathrm{H} & 2.38268000 & 2.13261400 & -0.01624400 \\ \mathrm{H} & 4.86247200 & 1.99152500 & -0.01335800 \\ \mathrm{~B} & 0.52162800 & 0.07010700 & -0.00145300 \\ \mathrm{C} & -2.07945600 & -0.49828200 & -0.00332000 \\ \mathrm{C} & -2.00624800 & 0.89889200 & 0.00211900 \\ \mathrm{C} & -3.32200000 & -1.13992300 & -0.00721100 \\ \mathrm{C} & -3.18350700 & 1.65357300 & 0.00352000 \\ \mathrm{C} & -4.49896300 & -0.39562100 & -0.00424100 \\ \mathrm{H} & -3.36554900 & -2.22579700 & -0.01257300 \\ \mathrm{C} & -4.41106300 & 1.00632500 & 0.00011700 \\ \mathrm{H} & -3.13267900 & 2.73801400 & 0.00587600 \\ \mathrm{H} & -5.32367100 & 1.59629200 & -0.00014000 \\ \mathrm{~S} & -0.55368500 & -1.38360800 & -0.00786500\end{array}$

$\begin{array}{llll}\mathrm{S} & -0.39864900 & 1.62519100 & 0.00500600 \\ \mathrm{C} & -5.84548800 & -1.07336600 & 0.00692900 \\ \mathrm{H} & -5.74677300 & -2.15120200 & -0.13924300 \\ \mathrm{H} & -6.35532100 & -0.90620600 & 0.96106800 \\ \mathrm{H} & -6.48930700 & -0.67773600 & -0.78381500 \\ \mathrm{~F} & 6.19584800 & -0.22252700 & 0.00273900\end{array}$

$G_{\mathrm{B} 3 \mathrm{LYP} / 6-311+\mathrm{G}(\mathrm{d}, \mathrm{p})}:-1422.633934$

$G_{\mathrm{M} 06-2 \mathrm{X} / 6-31+\mathrm{G}(\mathrm{d}, \mathrm{p})}:-1422.122572$

$G_{\mathrm{CBS-QB} 3}:-1381.419877$

$G_{\mathrm{MP} 2 / \text { aug-cc-pvdz: }}:-1380.463854$

\section{$14 \mathrm{e}$}

C $\quad-3.08843100 \quad-1.03718900 \quad-0.24063200$

$\begin{array}{lllll}\mathrm{C} & & -1.69801900 & -1.02331500 & -0.25538200\end{array}$

$\begin{array}{llll}\text { C } & -0.97769100 & 0.15559500 & 0.00094400\end{array}$

$\begin{array}{llll}\text { C } & -1.70314700 & 1.32804400 & 0.27493400\end{array}$

$\begin{array}{llll}\text { C } & -3.09254700 & 1.32370300 & 0.29736900\end{array}$

$\begin{array}{llll}\text { C } & -3.78755300 & 0.13990700 & 0.03939900\end{array}$

$\mathrm{H} \quad-3.63244300 \quad-1.95290300 \quad-0.44341600$

$\mathrm{H} \quad-1.16590800 \quad-1.94439100 \quad-0.47869100$

$\begin{array}{llll}\mathrm{H} & -1.17539300 & 2.25481600 & 0.48449400\end{array}$

$\begin{array}{llll}\mathrm{H} & -3.65153200 & 2.22789200 & 0.51504900\end{array}$

$\begin{array}{lllll}\text { B } & 0.57915200 & 0.16040600 & -0.01335200\end{array}$

$\begin{array}{llll}\text { C } & 3.14384200 & -0.53165600 & 0.05933900\end{array}$

$\begin{array}{lllll}\mathrm{C} & 3.13907900 & 0.85667300 & -0.11844500\end{array}$

$\begin{array}{llll}\text { C } & 4.35342800 & -1.22874900 & 0.14679800\end{array}$

$\begin{array}{lllll}\mathrm{C} & 4.35218000 & 1.54607000 & -0.21463400\end{array}$

$\begin{array}{llll}\mathrm{C} & 5.56484000 & -0.54938000 & 0.05349100\end{array}$

$\begin{array}{llll}\mathrm{H} & 4.34315600 & -2.30604900 & 0.28873700\end{array}$

$\begin{array}{llll}\mathrm{C} & 5.54574900 & 0.84404700 & -0.12781100\end{array}$

$\begin{array}{lllll}\mathrm{H} & 4.35496800 & 2.62259800 & -0.35460800\end{array}$

$\begin{array}{llll}\mathrm{H} & 6.48671300 & 1.38286000 & -0.20074600\end{array}$

S $\quad 1.57603800 \quad-1.33133300 \quad 0.17930600$

$\begin{array}{llll}\mathrm{S} & 1.56862300 & 1.65363400 & -0.22006300\end{array}$

$\begin{array}{llll}\mathrm{C} & 6.87759400 & -1.28601500 & 0.13637000\end{array}$

$\mathrm{H} \quad 6.72452100 \quad-2.34285300 \quad 0.36503900$

$\mathrm{H} \quad 7.42044000 \quad-1.21853800 \quad-0.81155100$

$\begin{array}{llll}\mathrm{H} & 7.51746900 & -0.85663600 & 0.91278500\end{array}$

$\begin{array}{llll}\text { C } & -5.27939400 & 0.17898100 & 0.07868800\end{array}$

$\begin{array}{llll}\text { O } & -5.92660100 & 1.17411600 & 0.32863300\end{array}$

$\begin{array}{lllll}\mathrm{O} & -5.83471800 & -1.00724700 & -0.18979400\end{array}$

$\begin{array}{llll}\text { C } & -7.26669600 & -1.04436400 & -0.16263400\end{array}$

$\mathrm{H} \quad-7.67417900 \quad-0.35235000 \quad-0.90159900$

$\mathrm{H} \quad-7.53721800 \quad-2.06990500 \quad-0.40423500$

$\begin{array}{llll}\mathrm{H} & -7.62977000 & -0.77245600 & 0.83012600\end{array}$

$G_{\mathrm{B} 3 \mathrm{LYP} / 6-311+\mathrm{G}(\mathrm{d}, \mathrm{p})}:-1551.268757$

$G_{\mathrm{M} 06-2 \mathrm{X} / 6-31+\mathrm{G}(\mathrm{d}, \mathrm{p})}:-1550.668909$

$G_{\mathrm{CBS}-\mathrm{QB} 3}:-1509.836664$

$G_{\mathrm{MP} 2 / \text { aug-cc-pvdz: }}:-1508.695476$ 


\begin{tabular}{|c|c|c|c|c|c|c|c|}
\hline \multirow[t]{2}{*}{$14 f$} & & & & $\mathrm{C}$ & 6.08576500 & -1.09828200 & 0.10321300 \\
\hline & & & & $\mathrm{H}$ & 5.97891100 & -2.16478800 & 0.31219700 \\
\hline $\mathrm{C}$ & -3.87904200 & -1.26002100 & -0.29307300 & $\mathrm{H}$ & 6.61441700 & -0.99006800 & -0.84900500 \\
\hline $\mathrm{C}$ & -2.49191400 & -1.18725200 & -0.29793200 & $\mathrm{H}$ & 6.71590000 & -0.65775600 & 0.88118900 \\
\hline $\mathrm{C}$ & -1.82231300 & 0.01367700 & -0.00870400 & C & -6.06156800 & -0.17960800 & 0.02146200 \\
\hline $\mathrm{C}$ & -2.59410800 & 1.14965600 & 0.28922600 & \multicolumn{4}{|c|}{$G_{\mathrm{B} 3 \mathrm{LYP} / 6-311+\mathrm{G}(\mathrm{d}, \mathrm{p})}:-1415.628272$} \\
\hline $\mathrm{C}$ & -3.98212600 & 1.09618700 & 0.30305900 & \multicolumn{4}{|c|}{$G_{\mathrm{M} 06-2 \mathrm{X} / 6-31+\mathrm{G}(\mathrm{d}, \mathrm{p})}:-1415.123487$} \\
\hline $\mathrm{C}$ & -4.62258900 & -0.11338900 & 0.01011900 & \multirow{2}{*}{\multicolumn{4}{|c|}{$G_{\mathrm{CBS}-\mathrm{QB} 3}:-1374.379566$}} \\
\hline $\mathrm{H}$ & -4.38740600 & -2.19080300 & -0.52018100 & & & & \\
\hline $\mathrm{H}$ & -1.92333500 & -2.08087400 & -0.54016200 & \multicolumn{4}{|c|}{$G_{\mathrm{MP} 2 / \text { aug-cc-pvdz }}:-1373.424532$} \\
\hline $\mathrm{H}$ & -2.10489200 & 2.09121900 & 0.52344900 & & & & \\
\hline $\mathrm{H}$ & -4.56908900 & 1.97708400 & 0.53930700 & & & & \\
\hline B & -0.26506200 & 0.08259100 & -0.01422900 & \multirow{2}{*}{\multicolumn{4}{|c|}{$\mathrm{H}_{2} \mathrm{O}$}} \\
\hline $\mathrm{C}$ & 2.32368700 & -0.50194100 & 0.04589600 & & & & \\
\hline $\mathrm{C}$ & 2.26004800 & 0.89050000 & -0.07927700 & $\mathrm{O}$ & 0.00000000 & 0.00000000 & 0.11657500 \\
\hline $\mathrm{C}$ & 3.56189400 & -1.14946800 & 0.11057600 & $\mathrm{H}$ & 0.00000000 & 0.76685900 & -0.46630200 \\
\hline $\mathrm{C}$ & 3.44235500 & 1.63506000 & -0.14300100 & $\mathrm{H}$ & 0.00000000 & -0.76685900 & -0.46630200 \\
\hline C & 4.74307500 & -0.41535100 & 0.04775900 & \multicolumn{4}{|c|}{$G_{\mathrm{B} 3 \mathrm{LYP} / 6-311+\mathrm{G}(\mathrm{d}, \mathrm{p})}:-76.460557$} \\
\hline $\begin{array}{l}\mathrm{H} \\
\mathrm{C}\end{array}$ & $\begin{array}{l}3.59809500 \\
4.66461400\end{array}$ & $\begin{array}{c}-2.23102600 \\
0.98214400\end{array}$ & $\begin{array}{r}0.20985000 \\
-0.07875800\end{array}$ & \multicolumn{4}{|c|}{$G_{\mathrm{M} 06-2 \mathrm{X} / 6-31+\mathrm{G}(\mathrm{d}, \mathrm{p})}:-76.397089$} \\
\hline $\mathrm{H}$ & 3.39884300 & 2.71539900 & -0.24053000 & \multicolumn{4}{|c|}{$G_{\text {СВS-ОВ3 }}:-76.360119$} \\
\hline $\mathrm{H}$ & 5.58160200 & 1.56340400 & -0.12582700 & \multirow{2}{*}{\multicolumn{4}{|c|}{$G_{\mathrm{MP} 2 / \text { aug-cc-pvdz }}:-76.262036$}} \\
\hline $\mathrm{S}$ & 0.79115100 & -1.37161200 & 0.12721200 & & & & \\
\hline & 0.65780800 & 1.62299200 & -0.15973500 & & & & \\
\hline
\end{tabular}




\section{Supporting References}

S1. Smith, M. K.; Northrop, B. H. Chem. Mater. 2014, 26, 3781-3795.

Complete citation for reference 50 of the main text:

Gaussian 09, Revision A.1, Frisch, M. J.; Trucks, G. W.; Schlegel, H. B.; Scuseria, G. E.; Robb, M. A.; Cheeseman, J. R.; Scalmani, G.; Barone, V.; Mennucci, B.; Petersson, G. A.; Nakatsuji, H.; Caricato, M.; Li, X.; Hratchian, H. P.; Izmaylov, A. F.; Bloino, J.; Zheng, G.; Sonnenberg, J. L.; Hada, M.; Ehara, M.; Toyota, K.; Fukuda, R.; Hasegawa, J.; Ishida, M.; Nakajima, T.; Honda, Y.; Kitao, O.; Nakai, H.; Vreven, T.; Montgomery, Jr., J. A.; Peralta, J. E.; Ogliaro, F.; Bearpark, M.; Heyd, J. J.; Brothers, E.; Kudin, K. N.; Staroverov, V. N.; Kobayashi, R.; Normand, J.; Raghavachari, K.; Rendell, A.; Burant, J. C.; Iyengar, S. S.; Tomasi, J.; Cossi, M.; Rega, N.; Millam, N. J.; Klene, M.; Knox, J. E.; Cross, J. B.; Bakken, V.; Adamo, C.; Jaramillo, J.; Gomperts, R.; Stratmann, R. E.; Yazyev, O.; Austin, A. J.; Cammi, R.; Pomelli, C.; Ochterski, J. W.; Martin, R. L.; Morokuma, K.; Zakrzewski, V. G.; Voth, G. A.; Salvador, P.; Dannenberg, J. J.; Dapprich, S.; Daniels, A. D.; Farkas, Ö.; Foresman, J. B.; Ortiz, J. V.; Cioslowski, J.; Fox, D. J. Gaussian, Inc., Wallingford CT, 2009. 